\title{
LINEAR DIFFERENTIAL POLYNOMIALS IN ZERO-FREE MEROMORPHIC FUNCTIONS
}

\author{
James K. Langley \\ University of Nottingham, School of Mathematical Sciences \\ NG7 2RD, United Kingdom; james.langley@nottingham.ac.uk \\ In fondest memory of Günter Frank and Milne Anderson
}

\begin{abstract}
The paper determines all meromorphic functions $f$ in $\mathbf{C}$ such that $f$ and $F$ have finitely many zeros, where $F=f^{(k)}+a_{k-1} f^{(k-1)}+\ldots+a_{0} f$ with $k \geq 3$ and the $a_{j}$ rational functions.
\end{abstract}

\section{Introduction}

Let the function $f$ be meromorphic in an annulus $\Omega\left(r_{1}\right)=\left\{z \in \mathbf{C}: r_{1}<|z|<\right.$ $\infty\}$, with $r_{1}$ positive (not necessarily the same at each occurrence in this paper). Let $k \geq 2$ and let $a_{0}, \ldots, a_{k-1}$ be functions which are rational at infinity, that is, analytic on some $\Omega\left(r_{1}\right)$ with at most a pole at $\infty$. Write $D=d / d z$ and

$$
F=L[f], \quad L=D^{k}+a_{k-1} D^{k-1}+\ldots+a_{0},
$$

in which $L[y]$ denotes the operator $L$ acting on the function $y$. The central objective of this paper is the classification of all those $f$ for which $f$ and $F$ have no zeros in $\Omega\left(r_{1}\right)$. By a standard change of variables $f=e^{P} g, F=e^{P} G$, with $P$ a polynomial, it may be assumed that $a_{k-1}(\infty)=0$.

This problem, part of which appeared as 1.42 in the collection [1], has a long history going back to Hayman's conjecture in [10], proved in [3, 18], that if $k \geq 2$ then the only meromorphic functions $f$ in the plane for which $f$ and $f^{(k)}$ have no zeros are those of form $f(z)=e^{a z+b}$ or $f(z)=(a z+b)^{-n}$ with $a, b \in \mathbf{C}$ and $n \in \mathbf{N}=\{1,2, \ldots\}$ : more generally, if $f$ and $f^{(k)}$ have finitely many zeros then $f=R e^{P}$, with $R$ a rational function and $P$ a polynomial $[5,18]$, so that $f^{\prime} / f$ is rational. The problem for $k=2$ and coefficients which are rational at infinity was fully solved in $[18,19]$.

Theorem 1.1. [18, 19] Let the function $f$ be meromorphic in $S \leq|z|<\infty$ for some $S>0$ and let the functions $a_{1}$ and $a_{0}$ be analytic there and rational at infinity. Assume that $a_{1}(\infty)=0$ and that $f$ and $F=f^{\prime \prime}+a_{1} f^{\prime}+a_{0} f$ have no zeros in $S \leq|z|<\infty$.

(a) If

$$
\operatorname{deg}_{\infty}\left(a_{0}\right)=\lim _{z \rightarrow \infty} \frac{\log \left|a_{0}(z)\right|}{\log |z|}
$$

is even then at least one of the following holds.

(i) The function $f^{\prime} / f$ is rational at infinity.

https://doi.org/10.5186/aasfm.2018.4348

2010 Mathematics Subject Classification: Primary 30D35.

Key words: Meromorphic function, zeros. 
(ii) The function $f$ satisfies

$$
\frac{f^{\prime}}{f}=-\frac{a_{1}}{2}+\frac{g^{\prime}}{2 g}+\frac{A}{g}, \quad g^{2}=\frac{f}{F}, \quad g^{\prime}=\left(2 \frac{f_{1}^{\prime}}{f_{1}}+a_{1}\right) g+B,
$$

where $A, B \in \mathbf{C}$ and $g$ is analytic in $|z| \geq S$, while $f_{1}$ is a solution of the homogeneous equation

$$
w^{\prime \prime}+a_{1} w^{\prime}+a_{0} w=0
$$

which admits unrestricted analytic continuation without zeros in $|z| \geq S$.

(iii) There exist solutions $f_{1}, f_{2}$ of (1.3), such that

$$
f=A f_{2}\left(1+B\left(\frac{f_{2}}{f_{1}}\right)^{1 / N}\right)^{-N}, \quad A, B \in \mathbf{C}, \quad N \in \mathbf{N} .
$$

Here both $f_{1}$ and $f_{2}$ admit unrestricted analytic continuation without zeros in $|z|>R_{1}$ for some $R_{1}>0$, and $\left(f_{2} / f_{1}\right)^{1 / N}$ is analytic in $|z|>R_{1}$.

(iv) There exist solutions $f_{1}, f_{2}$ of (1.3), each admitting unrestricted analytic continuation without zeros in $|z|>R_{1}$ for some $R_{1}>0$, a function $M$ which is rational at infinity, and non-constant polynomials $Q, Q_{1}$ such that

$$
\frac{f^{\prime}}{f}=\frac{f_{2}^{\prime}}{f_{2}}+\frac{Q(M) M^{\prime}}{e^{M}+1}, \quad \text { where } \quad Q(M) M^{\prime}=\frac{f_{1}^{\prime}}{f_{1}}-\frac{f_{2}^{\prime}}{f_{2}} \quad \text { or } \quad Q_{1}(M) e^{-M}=\frac{f_{1}}{f_{2}} .
$$

(b) If $\operatorname{deg}_{\infty}\left(a_{0}\right)$ is odd then $f$ may be determined by applying part (a) to

$$
\phi(z)=f\left(z^{2}\right), \quad \Phi(z)=4 z^{2} F\left(z^{2}\right)=\phi^{\prime \prime}(z)+\left(2 z a_{1}\left(z^{2}\right)-1 / z\right) \phi^{\prime}(z)+4 z^{2} a_{0}\left(z^{2}\right) \phi(z) .
$$

A refinement of this theorem for meromorphic functions in the plane may be found in [21, Theorem 1.3]. For $k \geq 3$ and $f, F$ zero-free in the whole plane, the case of constant coefficients was solved in full by Steinmetz in [23], while polynomial coefficients were treated in [4] for entire $f$, and for meromorphic $f$ by Brüggemann in [2].

Theorem 1.2. [2, 4] Let the function $f$ be meromorphic in the plane, such that $f$ and $F=L[f]$ have no zeros, where $k \geq 3$ and $a_{0}, \ldots, a_{k-2}$ are polynomials, not all constant, with $a_{k-1} \equiv 0$. Then $f=\left(H^{\prime}\right)^{-(k-1) / 2} e^{H}$ or $f=\left(H^{\prime}\right)^{-(k-1) / 2} H^{-m}$ for some $m \in \mathbf{N}$, where $H^{\prime \prime} / H^{\prime}$ is a polynomial.

The following theorem, which settles all cases, will be proved.

Theorem 1.3. Let $k \geq 3$ and let the function $f$ be meromorphic in some annulus $\Omega\left(r_{1}\right)$, with $f^{\prime} / f$ not rational at infinity. Assume that $f$ and $F=L[f]$ have no zeros in $\Omega\left(r_{1}\right)$, where $L$ is as in (1.1) with the $a_{j}$ analytic in $\Omega\left(r_{1}\right)$ and rational at infinity, and with $a_{k-1}(\infty)=0$. Then $f$ satisfies at least one of the following.

(i) The logarithmic derivative $f^{\prime} / f$ has a representation

$$
\frac{f^{\prime}}{f}=-\frac{a_{k-1}}{k}-\left(\frac{k-1}{2}\right) \frac{H^{\prime \prime}}{H^{\prime}}+H^{\prime} \quad \text { or } \quad \frac{f^{\prime}}{f}=-\frac{a_{k-1}}{k}-\left(\frac{k-1}{2}\right) \frac{H^{\prime \prime}}{H^{\prime}}-m \frac{H^{\prime}}{H},
$$

where $m \in \mathbf{N}$ and $H_{0}=H^{\prime \prime} / H^{\prime}$ is rational at infinity, with $H_{0}(\infty) \neq 0$, while the equation $L[y]=0$ has linearly independent local solutions $y_{j}$ satisfying

$$
\frac{y_{j}^{\prime}}{y_{j}}=-\frac{a_{k-1}}{k}-\left(\frac{k-1}{2}\right) \frac{H^{\prime \prime}}{H^{\prime}}+(j-1) \frac{H^{\prime}}{H}, \quad j=1, \ldots, k,
$$


and $f$ is given locally by either $f=c y_{1} \exp \left(y_{2} / y_{1}\right)$ or $f=c y_{1}^{m+1} y_{2}^{-m}$, where $c \in \mathbf{C} \backslash\{0\}$.

(ii) There exist a polynomial $Q$ and functions $\nu_{1}, \nu_{0}$, both rational at infinity, such that $f^{\prime} / f$ has a representation

$$
\frac{f^{\prime}}{f}=\frac{Q(T) T^{\prime}}{1-e^{-T}}+\frac{y_{1}^{\prime}}{y_{1}}, \quad T=\log \left(\frac{v}{u}\right),
$$

where $y_{1}$ is a solution of $L[y]=0$, while $v$ and $u$ are linearly independent solutions of

$$
y^{\prime \prime}+\nu_{1} y^{\prime}+\nu_{0} y=0
$$

which continue without zeros in some annulus $\Omega\left(r_{2}\right)$. Here $Q(T)$ is rational at infinity, and $u, v, y_{1}^{\prime} / y_{1}$ and $a_{0}, \ldots, a_{k-2}$ all have representations in terms of $Q(T), T, a_{k-1}$ and their derivatives. Moreover, if $T^{\prime}$ is not rational at infinity then $k$ is even and $z^{-1 / 2} T^{\prime}(z)$ is rational at infinity.

In both cases (i) and (ii) there exist $r_{3}>0$ and functions $\widetilde{a}_{1}, \widetilde{a}_{0}$, each rational at infinity, such that $f^{\prime \prime}+\widetilde{a}_{1} f^{\prime}+\widetilde{a}_{0} f$ has no zeros in $\Omega\left(r_{3}\right)$.

The conclusions of Theorems 1.1 and 1.3 are closely related, and the last assertion of Theorem 1.3 makes it clear that this is no coincidence. If $Q$ is a constant $d$ in (1.7) then integration shows that $f$ is a constant multiple of $y_{1}(v / u-1)^{d}$. Conclusion (1.5) may be compared with that of Theorem 1.2, and links closely to (1.2) of Theorem 1.1 and [21, Theorem 1.3(II)]. Examples II and III in Section 2 demonstrate that in (1.7) the multiplicities of poles of $f$ may be unbounded, in sharp contrast to the situation in Theorem 1.2, where any poles of $f$ must all have the same multiplicity $m$. Example III also shows that $T^{\prime}$ need not be rational at infinity in (1.7).

Some previous partial results for rational coefficients may be found in [13, 17]. Methods from $[2,3,4,23]$ are essential to the proof of Theorem 1.3; these are supplemented by a result (Lemma 3.1) on integer-valued analytic functions, facilitating the analytic continuation of several asymptotic representations. A decisive role is played by a criterion (Lemma 13.1) for certain auxiliary functions to satisfy a second order differential equation, which simplifies the subsequent analysis considerably.

The author acknowledges extensive discussions and correspondence on this problem with the late Günter Frank; these took place over many years and have contributed substantially to the methodology of this paper. Indeed, the Wronskianbased method invented by Frank $[3,5]$ underpins much of the successful work on these and related problems. Thanks are also due to the referees for their valuable comments.

\section{Examples}

Throughout the paper $c$ will be used to denote non-zero constants, not always the same at each occurrence, and $\mathbf{C}^{*}$ will denote $\mathbf{C} \backslash\{0\}$.

2.1. Example I. This example goes back to [4], and may be compared with conclusion (i) of Theorem 1.3 and that of Theorem 1.2. Let $H$ be such that $\delta=$ $H^{\prime \prime} / H^{\prime} \not \equiv 0$ is a polynomial, and write

$$
g=\left(H^{\prime}\right)^{-k} e^{H}, \quad h=\left(H^{\prime}\right)^{-k} H^{-m}, \quad D=\frac{d}{d z}, \quad m \in \mathbf{N} .
$$

Then it is easy to check (see the remark following (6.5) below) that

$$
(D+\delta) \ldots(D+k \delta)[g]=e^{H}, \quad(D+\delta) \ldots(D+k \delta)[h]=c H^{-m-k} .
$$


Taking $f$ to be $e^{P} g$ or $e^{P} h$ for a suitably chosen polynomial $P$ gives polynomial coefficients $a_{j}$ with $a_{k-1}=0$ such that $f$ and $F=L[f]$ have no zeros.

2.2. Example II. Let $P$ be a non-constant polynomial which takes positive integer values at all zeros of $1-e^{z}$, and write

$$
\frac{f^{\prime}(z)}{f(z)}=\frac{P(z)}{1-e^{z}}, \quad \frac{f^{\prime \prime}(z)}{f(z)}=\frac{Q_{1}(z) e^{z}+Q_{0}(z)}{\left(1-e^{z}\right)^{2}}, \quad Q_{1}=P-P^{\prime}, \quad Q_{0}=P^{\prime}+P^{2}
$$

Then $f$ is meromorphic and zero-free in the plane, with a pole of multiplicity $P(z)$ at a zero $z$ of $1-e^{z}$. A standard calculation yields polynomials $R_{j}$ such that

$$
\frac{f^{\prime \prime \prime}(z)}{f(z)}=\frac{R_{2}(z) e^{2 z}+R_{1}(z) e^{z}+R_{0}(z)}{\left(1-e^{z}\right)^{3}} .
$$

If $F=f^{\prime \prime \prime}+b_{2} f^{\prime \prime}+b_{1} f^{\prime}$, where the $b_{j}$ are rational functions, then

$$
\begin{aligned}
\frac{F(z)}{f(z)} & =\frac{B_{2}(z) e^{2 z}+B_{1}(z) e^{z}+B_{0}(z)}{\left(1-e^{z}\right)^{3}}, \\
B_{2} & =R_{2}-b_{2} Q_{1}+b_{1} P \\
B_{1} & =R_{1}+b_{2}\left(Q_{1}-Q_{0}\right)-2 b_{1} P, \\
B_{0} & =R_{0}+b_{2} Q_{0}+b_{1} P .
\end{aligned}
$$

Thus $F$ may be made zero-free in some $\Omega\left(r_{1}\right)$ by setting

$$
\begin{aligned}
0 & =R_{1}+b_{2}\left(Q_{1}-Q_{0}\right)-2 b_{1} P=R_{0}+b_{2} Q_{0}+b_{1} P, \\
\frac{F(z)}{f(z)} & =\frac{B_{2}(z) e^{2 z}}{\left(1-e^{z}\right)^{3}},
\end{aligned}
$$

these equations being solvable for $b_{1}$ and $b_{2}$, since $\left(Q_{1}-Q_{0}\right) P+2 Q_{0} P=\left(Q_{1}+Q_{0}\right) P \not \equiv$ 0 by (2.1). Similar calculations show that it is possible to achieve each of

$$
\frac{F(z)}{f(z)}=\frac{B_{1}(z) e^{z}}{\left(1-e^{z}\right)^{3}} ; \quad \frac{F(z)}{f(z)}=\frac{B_{0}(z)}{\left(1-e^{z}\right)^{3}} .
$$

Finally, should it be the case that $b_{2}(\infty) \neq 0$, there exist a polynomial $Q_{2}$ and rational functions $a_{j}$, with $a_{2}(\infty)=0$, such that writing $h=e^{Q_{2}} f$ gives

$$
\frac{F(z)}{f(z)}=\frac{f^{\prime \prime \prime}+b_{2} f^{\prime \prime}+b_{1} f^{\prime}}{f}=\frac{h^{\prime \prime \prime}+a_{2} h^{\prime \prime}+a_{1} h^{\prime}+a_{0} h}{h} .
$$

2.3. Example III. This is adapted from [19]. Let $Y(z)=z^{m / 2}$, where $m \in \mathbf{N}$, and set $h=\cosh Y$. Then $h$ is entire with only simple zeros. Let $P_{1}$ be an even polynomial which takes negative integer values at all odd integer multiples of $\pi i / 2$, and set $P=P_{1}(Y)$. Then $P$ is a polynomial and setting

$$
\frac{f^{\prime}}{f}=P \cdot \frac{h^{\prime}}{h}=\frac{P Y^{\prime} \sinh Y}{\cosh Y}=\frac{-2 P_{1}(Y) Y^{\prime}}{1+e^{2 Y}}+P_{1}(Y) Y^{\prime}
$$

defines $f$ as a meromorphic function in the plane, with no zeros. Next, set $R=$ $f^{\prime \prime}+b_{1} f^{\prime}+b_{0} f$, where $b_{1}=-P^{\prime} / P-Y^{\prime \prime} / Y^{\prime}$ and $b_{0}=-\left(P Y^{\prime}\right)^{2}$. This gives, since $h^{\prime \prime}=\left(Y^{\prime \prime} / Y^{\prime}\right) h^{\prime}+\left(Y^{\prime}\right)^{2} h$,

$$
\frac{R}{f}=\left(P-P^{2}\right)\left(\left(Y^{\prime}\right)^{2}-\left(\frac{h^{\prime}}{h}\right)^{2}\right)=\frac{\left(P-P^{2}\right)\left(Y^{\prime}\right)^{2}}{h^{2}},
$$


and so $R$ is zero-free in some $\Omega\left(r_{1}\right)$. Moreover, $S=R\left(P-P^{2}\right)^{-1}\left(Y^{\prime}\right)^{-2}$ satisfies $S / f=h^{-2}$ and $S^{\prime} / S=(P-2) h^{\prime} / h$. Hence the same construction, with $P$ replaced by $P-2$, gives rational functions $c_{j}, d_{j}$ and $e_{j}$ such that

$$
\frac{S^{\prime \prime}+c_{1} S^{\prime}+c_{0} S}{S}=\frac{R^{\prime \prime}+d_{1} R^{\prime}+d_{0} R}{R}=\frac{f^{(4)}+e_{3} f^{(3)}+\ldots+e_{0} f}{R}=\frac{F}{R}
$$

is free of zeros in some $\Omega\left(r_{2}\right)$, as is $F$.

\section{Preliminaries}

Lemma 3.1. Let the function $g$ be analytic on the half-plane $H^{+}$given by $\operatorname{Re} z \geq 0$, such that $g(n) \in \mathbf{Z}$ for all $n \in \mathbf{Z} \cap H^{+}$and $|g(z)|=o\left(2^{|z|}\right)$ as $z \rightarrow \infty$ in $H^{+}$. Then $g$ is a polynomial. Next, let $h(z)=e^{2 \pi i \alpha z} u(z)$, where $\alpha \in \mathbf{R}$ and $u$ is analytic on $H^{+}$, with $\log ^{+}|u(z)|=o(|z|)$ as $z \rightarrow \infty$ in $H^{+}$, and assume that $h(n)=1$ for all large $n \in \mathbf{N}$. Then $u(z) \equiv 1$ and $\alpha \in \mathbf{Z}$.

Proof. The first assertion is proved in [20]. To prove the second part let $\delta_{1} \in$ $(0, \infty)$ be small: then there exist $p \in \mathbf{Z}$ and $q \in \mathbf{N}$ such that

$$
\alpha=s+t, \quad s=\frac{p}{q}, \quad|t|<\frac{\delta_{1}}{2 \pi q} .
$$

Here $t=0$ if $\alpha$ is rational, while if $\alpha \notin \mathbf{Q}$ then suitable $p$ and $q$ exist by Dirichlet's approximation theorem [9, p. 155]. Write

$$
z=q w, \quad F(w)=e^{2 \pi i t q w} u(q w) .
$$

If $n \in \mathbf{N}$ is large then

$$
1=h(q n)=e^{2 \pi i \alpha q n} u(q n)=e^{2 \pi i(p n+t q n)} u(q n)=F(n) .
$$

Thus $F$ is a polynomial, by the first part, and so $F(w) \equiv 1$. Moreover, $t=0$, because otherwise there exists $\theta \in\{-\pi / 4, \pi / 4\}$ such that $F\left(r e^{i \theta}\right) \rightarrow 0$ as $r \rightarrow+\infty$, and so $u \equiv 1$. Finally, $\alpha=p / q$ must be an integer, since $1=h(q n+1)=\exp (2 \pi i p / q)$ for large $n \in \mathbf{N}$.

Lemma 3.2. Let $d_{1}, d_{2}$ and $\lambda$ be positive constants and let $g$ be a zero-free analytic function on the half-plane $\operatorname{Re}(w)>0$, with $\log ^{+}|g(w)| \leq d_{1}+d_{2}|w|^{\lambda}$ there. Then for $0<\alpha<\pi / 2$ there exists $\mu=\mu_{\alpha}>0$ such that $\log ^{+}|1 / g(w)| \leq \mu_{\alpha}|w|^{1+\lambda}$ as $w \rightarrow \infty$ with $|\arg w| \leq \alpha$.

Proof. This is standard: set $w=(1+z) /(1-z)$ and $g(w)=G(z)$ for $|z|<1$. With $\rho=(1+r) / 2$ this leads to

$$
\begin{aligned}
\log M(r, 1 / G) & \leq\left(\frac{\rho+r}{\rho-r}\right) T(\rho, 1 / G) \leq\left(\frac{\rho+r}{\rho-r}\right)(\log M(\rho, G)+O(1)) \\
& =O(1-r)^{-1-\lambda}
\end{aligned}
$$

as $r \rightarrow 1-$. It remains only to observe that there exists $c_{1}=c_{1}(\alpha)>0$ such that if $|w|$ is large and $|\arg w| \leq \alpha<\pi / 2$ then $\left(1-|z|^{2}\right)^{-1} \leq c_{1}|w|$.

Lemma 3.3. Suppose that $p$ and $q$ are (both formal or both locally analytic) solutions of the equations

$$
\frac{p^{\prime}}{p}=d_{0} \frac{q^{\prime}}{q}+d_{1}, \quad q^{\prime \prime}+\nu_{1} q^{\prime}+\nu_{0} q=0,
$$


where the $d_{j}$ and $\nu_{j}$ are rational at infinity, and let $L$ be as in (1.1). Then there exist coefficients $b_{j}$, each rational at infinity, such that $p$ and $q$ satisfy

$$
\frac{L[p]}{p}=\sum_{j=0}^{k} b_{j}\left(\frac{q^{\prime}}{q}\right)^{j}, \quad b_{k}=d_{0}\left(d_{0}-1\right) \ldots\left(d_{0}-k+1\right) .
$$

Moreover, if $d_{0} \not \equiv 0,1$ and $e_{1}$ and $e_{0}$ are rational at infinity, then there exist coefficients $E_{\mu}$, each rational at infinity and depending only on the $d_{j}, e_{j}$ and $\nu_{j}$, such that $E_{2} \not \equiv 0$ and

$$
E_{2} \frac{p^{\prime \prime}}{p}+E_{1} \frac{p^{\prime}}{p}+E_{0}=\left(\frac{p^{\prime}}{p}\right)^{2}+e_{1} \frac{p^{\prime}}{p}+e_{0}
$$

Proof. Formula (3.2) follows from (3.1) and a simple induction argument, which deliver

$$
\left(\frac{q^{\prime}}{q}\right)^{\prime}=-\left(\frac{q^{\prime}}{q}\right)^{2}-\nu_{1}\left(\frac{q^{\prime}}{q}\right)-\nu_{0}, \quad \frac{p^{(m)}}{p}=\sum_{j=0}^{m} b_{j, m}\left(\frac{q^{\prime}}{q}\right)^{j}, \quad m \in \mathbf{N}
$$

with the $b_{j, m}$ rational at infinity and $b_{m, m}=d_{0}\left(d_{0}-1\right) \ldots\left(d_{0}-m+1\right)$.

To prove the second part, suppose that $d_{0} \not \equiv 0,1$ and write $P=p^{\prime} / p$ and $Q=q^{\prime} / q$ so that $Q=A P+B$, with $A, B$ rational at infinity and $A \not \equiv 0,1$. This yields

$$
\begin{aligned}
0 & =A^{\prime} P+A P^{\prime}+B^{\prime}+A^{2} P^{2}+2 A B P+B^{2}+\nu_{1}(A P+B)+\nu_{0} \\
& =\left(A^{2}-A\right) P^{2}+A\left(P^{\prime}+P^{2}\right)+\left(A^{\prime}+2 A B+\nu_{1} A\right) P+B^{\prime}+B^{2}+\nu_{1} B+\nu_{0},
\end{aligned}
$$

and so

$$
\begin{aligned}
\left(A-A^{2}\right)\left(P^{2}+e_{1} P+e_{0}\right)= & A\left(P^{\prime}+P^{2}\right)+\left(A^{\prime}+2 A B+\nu_{1} A+\left(A-A^{2}\right) e_{1}\right) P \\
& +B^{\prime}+B^{2}+\nu_{1} B+\nu_{0}+\left(A-A^{2}\right) e_{0} .
\end{aligned}
$$

Lemma 3.4. Suppose that $u$ and $v$ are linearly independent (both formal or both locally analytic) solutions of an equation

$$
y^{\prime \prime \prime}+B_{2} y^{\prime \prime}+B_{1} y^{\prime}+B_{0} y=0
$$

with the $B_{j}$ rational at infinity, and assume that $W=W(u, v)=u v^{\prime}-u^{\prime} v$ is such that $W^{\prime}+E_{1} W=0$, where $E_{1}$ is rational at infinity. Then $u$ and $v$ solve an equation

$$
y^{\prime \prime}+E_{1} y^{\prime}+E_{0} y=0,
$$

where $E_{0}$ is also rational at infinity.

Proof. Since $u$ and $v$ are solutions of the equation $W(u, v, y)=0$, it is enough to prove that $W\left(u^{\prime}, v^{\prime}\right)=E_{2} W(u, v)$ with $E_{2}$ rational at infinity. But $W^{\prime}=u v^{\prime \prime}-u^{\prime \prime} v=$ $-E_{1} W$ leads to

$$
\begin{aligned}
\left(-E_{1}^{\prime}+E_{1}^{2}\right) W & =W^{\prime \prime}=u^{\prime} v^{\prime \prime}-u^{\prime \prime} v^{\prime}+u v^{\prime \prime \prime}-u^{\prime \prime \prime} v \\
& =W\left(u^{\prime}, v^{\prime}\right)+v\left(B_{2} u^{\prime \prime}+B_{1} u^{\prime}+B_{0} u\right)-u\left(B_{2} v^{\prime \prime}+B_{1} v^{\prime}+B_{0} v\right) \\
& =W\left(u^{\prime}, v^{\prime}\right)-B_{2} W^{\prime}-B_{1} W=W\left(u^{\prime}, v^{\prime}\right)+\left(E_{1} B_{2}-B_{1}\right) W .
\end{aligned}
$$

\section{Asymptotics for linear differential equations}

As in [2] a fundamental role will be played by formal and asymptotic expansions for solutions of linear differential equations. For an equation $L[y]=0$, with $L$ as in (1.1) and the $a_{j}$ rational at infinity, classical results (see [24, Theorem 19.1] or 
$[2,16]))$ show that there exist $p \in \mathbf{N}$ and a fundamental set of $k$ linearly independent formal solutions

$$
\widetilde{h}_{j}(z)=\exp \left(P_{j}\left(z^{1 / p}\right)\right) z^{\gamma_{j}} \sum_{\mu=0}^{n_{j}} U_{j, \mu}\left(z^{1 / p}\right)(\log z)^{\mu}
$$

which satisfy the following: $\gamma_{j}$ is a complex number; $n_{j}$ is a non-negative integer; the exponential part $P_{j}\left(z^{1 / p}\right)$ is a polynomial in $z^{1 / p}$; the $U_{j, \mu}\left(z^{1 / p}\right)$ are formal series in descending integer powers of $z^{1 / p}$, that is, in which at most finitely many positive powers occur; the lead series $U_{j, n_{j}}$ is not the zero series. Formal solutions (not necessarily linearly independent) with these properties will be referred to henceforth as canonical formal solutions.

A standard approach [24] to obtaining these $\widetilde{h}_{j}(z)$ is to transform a solution $h$ of $L[y]=0$ into a vector $\mathbf{h}=\left(h, h^{\prime}, \ldots, h^{(k-1)}\right)$, so that a fundamental solution set for $L[y]=0$ corresponds to the first row of a matrix solution $V(z)=U(z) z^{G} e^{Q(z)}$ of an equation $Y^{\prime}=A(z) Y$, where $Q(z)$ is a diagonal matrix, its entries polynomials in $z^{1 / p}$, while $G$ is a constant matrix, which may be assumed to be in Jordan form, and $U(z)$ is a matrix with entries which are formal series in descending integer powers of $z^{1 / p}$. Furthermore, for each $\theta \in \mathbf{R}$ there exists $\delta=\delta(\theta)>0$ such that $L[y]=0$ has a fundamental set of analytic solutions

$$
h_{j}(z)=\exp \left(P_{j}\left(z^{1 / p}\right)\right) z^{\gamma_{j}} \sum_{\mu=0}^{n_{j}} V_{j, \mu}\left(z^{1 / p}\right)(\log z)^{\mu}
$$

on a sector $S$ given by $|z|>R_{0}>0,|\arg z-\theta|<\delta$, in which each $V_{j, \mu}\left(z^{1 / p}\right)$ is analytic on $S$ and satisfies $V_{j, \mu}\left(z^{1 / p}\right) \sim U_{j, \mu}\left(z^{1 / p}\right)$ as $z \rightarrow \infty$ on $S$, in the sense of asymptotic series (see [24, Theorem 19.1] or [22]). Here $W(z) \sim U(z)=\sum_{m=M}^{\infty} U_{m} z^{-m / p}$ as $z \rightarrow \infty$ on $S$ means that, for each $n \geq M$,

$$
W(z)-\sum_{m=M}^{n} U_{m} z^{-m / p}=o\left(|z|^{-n / p}\right) \quad \text { as } z \rightarrow \infty \text { in } S .
$$

It may be assumed that the exponential parts $P_{j}\left(z^{1 / p}\right)$ have zero constant term, and this convention will be used throughout. Given any exponential part $P_{j}\left(z^{1 / p}\right)$ arising for $L[y]=0$, there is always a canonical formal solution with exponential part $P_{j}\left(z^{1 / p}\right)$ which is free of logarithms, that is, has $n_{j}=0$; this holds because the matrix $G$ may be chosen to be in Jordan form. The following lemma is well known $[2,16,24]$.

Lemma 4.1. Given $k$ linearly independent canonical formal solutions of $L[y]=$ 0 with exponential parts $q_{1}, \ldots, q_{k}$, their formal Wronskian has exponential part $\sum_{j=1}^{k} q_{j}$, and the exponential parts of any fundamental set of canonical formal solutions of $L[y]=0$ form a permutation of the $q_{j}$.

For the special case of a second order equation, suppose that $A^{*}$ is rational at infinity, with $A^{*}(z)=(1+o(1)) c_{n} z^{n}$ as $z \rightarrow \infty$, where $c_{n} \in \mathbf{C}^{*}$ and $n \geq-1$. Then infinity is an irregular singular point for

$$
w^{\prime \prime}+A^{*} w=0
$$

and asymptotics are developed via Hille's method [14] as follows. The critical rays are given by $\arg z=\theta^{*}$, where $c_{n} e^{i(n+2) \theta^{*}}$ is real and positive. If $0<\beta<2 \pi /(n+2)$ 
then, in a sector given by $|z|>r_{1},\left|\arg z-\theta^{*}\right|<\beta$, there exist linearly independent analytic solutions, for $j=1,2$,

$$
\begin{aligned}
\phi_{j}(z) & =A^{*}(z)^{-1 / 4}(1+o(1)) \exp \left((-1)^{j} i Z\right), \\
Z & =\int^{z} A^{*}(t)^{1 / 2} d t=\frac{2 c_{n}^{1 / 2} z^{(n+2) / 2}}{n+2}+\ldots
\end{aligned}
$$

If $n=-1$ then this sector should be understood as lying on the Riemann surface of $\log z$. To one side of the critical ray, one of these solutions is large and the other small, and these roles are reversed as the critical ray is crossed. Any linear combination $D_{1} \phi_{1}+D_{2} \phi_{2}$ with $D_{1}, D_{2} \in \mathbf{C}^{*}$ has a sequence of zeros tending to infinity near the critical ray. Moreover, the corresponding formal solutions, to which the $\phi_{j}$ are asymptotic, may be calculated readily from $A^{*}(z)$ and $Z$, with $n_{j}=0$ and $p \in\{1,2\}$ in (4.1) (see [19] for details).

Lemma 4.2. Suppose that $F_{1}, \ldots, F_{k}$ are formal expressions, each of which is given by

$$
F_{j}(z)=U_{j}(z) z^{\gamma_{j}} r_{j}(z) \exp \left(q_{j}(z)\right)
$$

with $\gamma_{j} \in \mathbf{C}, r_{j}$ a rational function, $q_{j}$ a polynomial and $U_{j}(z)=1+O(1 / z)$, in which $O(1 / z)$ denotes a formal series in negative integer powers of $z$. Assume that none of the $r_{j}$ vanishes identically, and that $q_{j}-q_{j^{\prime}}$ is non-constant for $j \neq j^{\prime}$. Then the formal Wronskian $W=W\left(F_{1}, \ldots, F_{k}\right)$ has an expansion

$$
W(z)=\left(1+O\left(\frac{1}{z}\right)\right)\left(\prod_{j=1}^{k}\left[z^{\gamma_{j}} r_{j}(z) \exp \left(q_{j}(z)\right)\right]\right)\left(\prod_{k \geq m>n \geq 1}\left[q_{m}^{\prime}(z)-q_{n}^{\prime}(z)\right]\right) .
$$

Proof. This is standard, and is proved by induction on $k$, using

$$
\begin{aligned}
W\left(F_{1}, \ldots, F_{k}\right) & =F_{1}^{k} W\left(1, \frac{F_{2}}{F_{1}}, \ldots, \frac{F_{k}}{F_{1}}\right)=F_{1}^{k} W\left(\left(\frac{F_{2}}{F_{1}}\right)^{\prime}, \ldots,\left(\frac{F_{k}}{F_{1}}\right)^{\prime}\right), \\
\left(\frac{F_{j}}{F_{1}}\right)^{\prime}(z) & =\left(1+O\left(\frac{1}{z}\right)\right) z^{\gamma_{j}-\gamma_{1}}\left(\frac{r_{j}(z)}{r_{1}(z)}\right)\left(q_{j}^{\prime}(z)-q_{1}^{\prime}(z)\right) \exp \left(q_{j}(z)-q_{1}(z)\right) .
\end{aligned}
$$

\section{Beginning the proof of Theorem 1.3: Frank's method}

Assume that $f$ is as in the hypotheses of Theorem 1.3. Let $f_{1}, \ldots, f_{k}$ be linearly independent locally analytic solutions of $L[y]=0$. Frank's method [3, 4] defines $g, h, w_{j}$ and $Y$ locally by

$$
g^{k}=\frac{f}{F}, \quad h=-\left(\frac{f^{\prime}}{f}\right) g, \quad w_{j}=f_{j}^{\prime} g+f_{j} h=f g\left(\frac{f_{j}}{f}\right)^{\prime}, \quad \frac{Y^{\prime}}{Y}=-a_{k-1} .
$$

Note that $g$ might not be meromorphic in $\Omega\left(r_{1}\right)$, but $g^{\prime} / g$ is, and has a simple pole with residue 1 at every pole of $f$; moreover, at a pole of $f$ of multiplicity $m_{0}$, calculating the leading Laurent coefficient of $F / f$ gives

$$
\left(g^{\prime}\right)^{-k}=(-1)^{k} m_{0}\left(m_{0}+1\right) \cdots\left(m_{0}+k-1\right) .
$$

Now write locally, using Abel's identity, $W\left(f_{1}, \ldots, f_{k}\right)=c Y$ and

$$
\frac{Y}{(f g)^{k}}=\frac{Y F}{f^{k+1}}=\frac{c W\left(f_{1}, \ldots, f_{k}, f\right)}{f^{k+1}}=c W\left(\frac{f_{1}}{f}, \ldots, \frac{f_{k}}{f}, 1\right)=c W\left(\left(\frac{f_{1}}{f}\right)^{\prime}, \ldots,\left(\frac{f_{k}}{f}\right)^{\prime}\right)
$$


so that $Y=c W\left(w_{1}, \ldots, w_{k}\right)$ by $(5.1)$. Hence the $w_{j}$ are linearly independent (local) solutions of an equation $M[y]=0$, where

$$
M=D^{k}+A_{k-1} D^{k-1}+A_{k-2} D^{k-2}+\ldots+A_{0}, \quad A_{k-1}=a_{k-1}, \quad D=\frac{d}{d z} .
$$

Here a pivotal role is played by whether or not the differential operators $L$ and $M$ are the same, and Brüggemann's method in [2] depends on reducing the problem to the case $L=M$. It will be proved in Proposition 6.1 below that if $L=M$ then all poles $z$ of $f$ with $|z|$ sufficiently large have the same multiplicity. Thus Example II in Section 2 demonstrates that $L$ and $M$ can indeed be different operators.

By Frank's method, the $A_{j}$ are analytic in some annulus $\Omega\left(r_{1}\right)$ and satisfy $T\left(r, A_{j}\right)=S\left(r, f^{\prime} / f\right)$, where $S\left(r, f^{\prime} / f\right)$ denotes any term which is $O\left(\log T\left(r, f^{\prime} / f\right)+\right.$ $\log r)$ as $r \rightarrow \infty$, possibly outside a set of finite measure [11]: see [6, Section 2 and Lemmas A, B and 5] for details, including the Nevanlinna characteristic in $\Omega\left(r_{1}\right)$. Denote by $\Lambda$ the field generated by the $a_{j}, A_{j}$ and their derivatives: then $T(r, \lambda)=S\left(r, f^{\prime} / f\right)$ for all $\lambda \in \Lambda$.

To simplify the subsequent calculations it is convenient to write

$$
\begin{aligned}
-k \frac{X^{\prime}}{X} & =a_{k-1}=A_{k-1}, \quad p=\frac{f}{X}, \quad p_{j}=\frac{f_{j}}{X}, \\
q & =-\left(\frac{p^{\prime}}{p}\right) g, \quad t_{j}=p_{j}^{\prime} g+p_{j} q=\frac{w_{j}}{X} .
\end{aligned}
$$

It is then well known that there exist equations

$$
y^{(k)}+c_{k-2} y^{(k-2)}+\ldots+c_{0} y=0,
$$

and

$$
y^{(k)}+C_{k-2} y^{(k-2)}+\ldots+C_{0} y=0,
$$

in which the $c_{j}$ and $C_{j}$ all belong to $\Lambda$, and $c_{k-2}=C_{k-2}$ if and only if $a_{k-2}=A_{k-2}$, such that $L[X y]=0$ if and only if $y$ solves (5.5), and $M[X y]=0$ if and only if $y$ solves (5.6). In particular, the $p_{j}$ and $t_{j}$ are linearly independent local solutions of (5.5) and (5.6) respectively. The following lemma [4] is key to Frank's method: see also [6, Lemma C].

Lemma 5.1. [4] Let $G, \Phi, p_{1}, \ldots, p_{k}, c_{0}, \ldots, c_{k-2}$ and $C_{0}, \ldots, C_{k-2}$ be analytic functions on a plane domain $U$, such that $p_{1}, \ldots, p_{k}$ are linearly independent solutions of (5.5). Then the functions $p_{1}^{\prime} G+p_{1} \Phi, \ldots, p_{k}^{\prime} G+p_{k} \Phi$ are solutions in $U$ of the equation (5.6) if and only if, with the notation $C_{k}=1$ and $c_{k-1}=C_{k-1}=c_{-1}=$ $C_{-1}=0$ and

$$
M_{k, \mu}[w]=\sum_{m=\mu}^{k} \frac{m !}{\mu !(m-\mu) !} C_{m} w^{(m-\mu)} \quad(0 \leq \mu \leq k), \quad M_{k,-1}[w]=0,
$$

the functions $G$ and $\Phi$ satisfy, for $0 \leq \mu \leq k-1$,

$$
M_{k, \mu}[\Phi]-c_{\mu} \Phi=-M_{k, \mu-1}[G]+c_{\mu} M_{k, k-1}[G]+\left(c_{\mu}^{\prime}+c_{\mu-1}\right) G .
$$

Because the proof of Lemma 5.1 is based on purely formal calculations, an analogous statement holds linking formal solutions of (5.5), (5.6) and (5.7). Since the coefficient of $\Phi$ in $M_{k, \mu}[\Phi]-c_{\mu} \Phi$ is $c_{0, \mu}=D_{\mu}=C_{\mu}-c_{\mu}$, the equations (5.7) may be 
written in the form

$$
T_{\mu}[G]=S_{\mu}[\Phi]=\sum_{j=0}^{k-\mu} c_{j, \mu} \Phi^{(j)}, \quad c_{0, \mu}=D_{\mu}=C_{\mu}-c_{\mu}, \quad 0 \leq \mu \leq k-1,
$$

in which $T_{\mu}$ and $S_{\mu}$ are linear differential operators with coefficients in $\Lambda$. In particular these equations are satisfied by $G=g, \Phi=q$. Taking $\mu=k-1$ in (5.7) produces

$$
-\Phi^{\prime}=-U[G]=\frac{(k-1) G^{\prime \prime}}{2}+\frac{D_{k-2} G}{k} .
$$

Now $\mu=k-2$ and (5.9) give (as in [7, p. 162] or [17, Lemma 8, pp. 307-8])

$D_{k-2} \Phi=\frac{k\left(k^{2}-1\right)}{12} G^{\prime \prime \prime}+G^{\prime}\left(-\frac{(k+1) D_{k-2}}{2}+2 C_{k-2}\right)+G\left(\frac{k-1}{2} D_{k-2}^{\prime}+c_{k-2}^{\prime}-D_{k-3}\right)$.

Next, combining (5.9) with (5.7) for $\mu=k-3$ yields, with $d_{j}$ denoting elements of $\Lambda$,

$$
\frac{2 D_{k-3}}{k-2} \Phi=\frac{k\left(k^{2}-1\right)}{12} G^{(4)}+G^{\prime \prime}\left(\frac{(k-1) D_{k-2}}{3}+2 C_{k-2}\right)+d_{1} G^{\prime}+d_{2} G
$$

Note that (5.11) holds even if $k=3$, in which case $M_{k, k-4}[G]=0$. Differentiating (5.10) and using (5.9) and (5.11) leads to

$$
D^{*} \Phi=\left(\frac{2 D_{k-3}}{k-2}-D_{k-2}^{\prime}\right) \Phi=\frac{(k+2) D_{k-2}}{3} G^{\prime \prime}+d_{3} G^{\prime}+d_{4} G .
$$

Lemma 5.2. There exists a non-trivial homogeneous linear differential equation $N_{1}[y]=0$, of order at most 3 and with coefficients in $\Lambda$, with the property that if the pair $\{G, \Phi\}$ solves the system (5.8) then $G$ solves $N_{1}[y]=0$.

Proof. If $D_{k-2} \equiv 0$ this is clear from (5.10), so assume that $D_{k-2} \not \equiv 0$. If $D^{*}$ vanishes identically in (5.12) then a second order equation arises for $G$, while otherwise combining (5.12) with (5.10) yields a third order equation.

Consider now two cases.

Case 1. Assume that $c_{0, \mu}=C_{\mu}-c_{\mu} \equiv 0$ for $0 \leq \mu \leq k-1$ in (5.8). This is equivalent to the equations (5.5) and (5.6) being the same, and hence equivalent to the operators $L$ and $M$ being identical. In this case, $t_{j}=p_{j}^{\prime} g+p_{j} q$ is a solution of (5.5) for $1 \leq j \leq k$. Since $p_{1}$ and $p_{2}$ are linearly independent, $p_{1} p_{2}^{\prime}-p_{1}^{\prime} p_{2}$ does not vanish identically and so (5.4) yields

$$
H_{1}=\frac{f^{\prime}}{f}+\frac{a_{k-1}}{k}=\frac{p^{\prime}}{p}=-\frac{q}{g}=\frac{p_{1}^{\prime} t_{2}-p_{2}^{\prime} t_{1}}{p_{1} t_{2}-p_{2} t_{1}}
$$

For $\theta \in \mathbf{R}$ and $\kappa \in \mathbf{C}$ the number of distinct zeros of $\kappa-H_{1}$ in $r_{1}+1 \leq|z| \leq$ $r,|\arg z-\theta| \leq \pi / 4$, is at most the number of zeros of $p_{1}^{\prime} t_{2}-p_{2}^{\prime} t_{1}-\kappa\left(p_{1} t_{2}-p_{2} t_{1}\right)$ there, which is bounded by a power of $r$ as $r \rightarrow \infty$, by [6, Lemma 2] or standard sectorial methods. Hence $f^{\prime} / f$ has finite order of growth, by the second fundamental theorem, and every $\lambda \in \Lambda$ is rational at infinity.

Case 2. Assume that the coefficient of $\Phi$ in at least one of the $S_{\mu}$ in (5.8) is not identically zero, this being equivalent to $L \neq M$. 
Let $\nu$ be the largest integer with $0 \leq \nu \leq k-1$ such that $c_{0, \nu} \not \equiv 0$. Then every pair $\{G, \Phi\}$ satisfying the system (5.8) (including $\{g, q\}$ ) has

$$
\Phi=\left(c_{0, \nu}\right)^{-1}\left(T_{\nu}[G]-\sum_{j=1}^{k-\nu} c_{j, \nu} \frac{d^{j-1}}{d z^{j-1}}(U[G])\right)=T^{*}[G],
$$

by (5.9). Observe that the operator $T^{*}$ has order at least 1 , and in particular is not the zero operator, since otherwise (5.4) leads to $\left(-p^{\prime} / p\right) g=q=\eta_{1} g$, with $\eta_{1} \in \Lambda$, so that $f^{\prime} / f \in \Lambda$, and hence $f^{\prime} / f$ is rational at infinity, contrary to assumption. It follows from (5.8), (5.9) and (5.13) that if $\{G, \Phi\}$ solves (5.8) then $G$ solves the system

$$
U[G]=\frac{d}{d z}\left(T^{*}[G]\right), \quad S_{\mu}\left(T^{*}[G]\right)=T_{\mu}[G], \quad 0 \leq \mu \leq k-2,
$$

as does, in particular, $g$. Conversely, if $G$ solves the system (5.14) (in the analytic or formal sense), then (5.8) is satisfied by setting $\Phi=T^{*}[G]$. This system (5.14) cannot be trivial, because otherwise (5.8) holds with $\Phi=T^{*}[G]$ and an arbitrary choice of $G$, which would then have to solve the equation $N_{1}[y]=0$ of Lemma 5.2. A standard reduction procedure [15, p.126] now generates a non-trivial homogeneous linear differential equation $N[y]=0$, with coefficients in the field $\Lambda$, whose (analytic or formal) solution space coincides with that of the system (5.14). Here every solution $G$ of $N[y]=0$ is such that the pair $\left\{G, T^{*}[G]\right\}$ solves (5.8), and so $G$ solves $N_{1}[y]=0$, from which it follows that $N$ has order at most 3 .

Suppose that $N$ has order 1: then $g^{\prime} / g \in \Lambda$, and so $p^{\prime} / p$ and $f^{\prime} / f$ belong to $\Lambda$, by (5.4) and (5.13), so that $f^{\prime} / f$ is rational at infinity, contrary to assumption. Thus $N$ has order at least 2 , but at most 3, and the system (5.14) has a solution $G$ with $G / g$ non-constant. By an argument from [4] (see [6, Proof of Theorem 3, Case 1B] for details), $p^{\prime} / p$ has a representation as a rational function in the $p_{j}$ and their derivatives. The same sectorial argument as used in Case 1 shows that $f^{\prime} / f$ has finite order of growth, as has $g^{k}=f / F$, and all members of the field $\Lambda$ are rational at infinity.

Hence the fact that $N$ has order at most 3 gives an operator $V_{2}$, having order at most 2 and coefficients which are rational at infinity, with the following property. Every solution $G$ of (5.14) has $T^{*}[G]=V_{2}[G]$, so that the pair $\left\{G, V_{2}[G]\right\}$ solves (5.8), and $p_{j}^{\prime} G+p_{j} V_{2}[G]$ solves (5.6) for $j=1, \ldots, k$, by Lemma 5.1. Moreover, (5.13) gives $q=T^{*}[g]=V_{2}[g]$. With $V=V_{2}+a_{k-1} / k$, this implies using (5.4) that each $f_{j}^{\prime} G+f_{j} V[G]$ solves $M[y]=0$, and $-f^{\prime} / f=h / g=V[g] / g$. It now follows, using the Wiman-Valiron theory [12] and estimates for logarithmic derivatives [8] applied to $g$, that $f$ has finite order. It also follows that $f$ has an unbounded sequence of poles, since otherwise $f^{\prime} / f$ is rational at infinity. The following key lemma has thus been proved.

Lemma 5.3. With the hypotheses of Theorem 1.3, the function $f^{\prime} / f$ has finite order, all elements of the field $\Lambda$ are rational at infinity, and $h=-\left(f^{\prime} / f\right) g$ satisfies

$$
-h^{\prime}=\left(\frac{k-1}{2}\right) g^{\prime \prime}-\frac{a_{k-1}}{k} g^{\prime}+\frac{D_{k-2}-a_{k-1}^{\prime}}{k} g, \quad D_{j}=C_{j}-c_{j} .
$$

Furthermore, if the operators $L$ and $M$ are not the same then the following additional conclusions hold. The function $g$ solves a homogeneous linear differential equation $N[y]=0$, of order 2 or 3 , with coefficients which are rational at infinity. Moreover, 
$f$ has finite order and an unbounded sequence of poles, and there exist functions $\alpha, \beta, \gamma$, all rational at infinity, such that

$$
h=-\left(\frac{f^{\prime}}{f}\right) g=V[g], \quad V=\alpha D^{2}+\beta D+\gamma .
$$

Finally, if $G$ is a locally analytic solution of $N[y]=0$, and $K$ is a locally analytic solution of $L[y]=0$, then $K^{\prime} G+K V[G]$ is a (possibly trivial) solution of $M[y]=$ 0 .

Here (5.15) follows from (5.4) and (5.9). The last assertion of Lemma 5.3 also holds for formal solutions $G$ and $K$ of $N[y]=0$ and $L[y]=0$ respectively.

\section{The first special case}

Proposition 6.1. With the hypotheses of Theorem 1.3, suppose in addition that $c_{k-2}=C_{k-2}$ in (5.5) and (5.6), which holds if and only if $a_{k-2}=A_{k-2}$ in $L$ and $M$, and certainly holds if the operators $L$ and $M$ are the same. Then $f$ satisfies conclusion (i) of Theorem 1.3.

Proof. The approach here is essentially due to Frank and Hellerstein [4]. Since $D_{k-2}=0$ in (5.15), integration gives a constant $d$ such that

$$
\frac{f^{\prime}}{f}=-\frac{h}{g}=\frac{(k-1) g^{\prime}}{2 g}+\frac{d}{g}-\frac{a_{k-1}}{k} .
$$

If $d=0$ then comparing residues shows that $f$ has no poles in some $\Omega\left(r_{2}\right)$ and $F / f$, which has finite order by Lemma 5.3, satisfies $g^{-k}=F / f=R_{1} e^{P_{1}}$ with $R_{1}$ rational at infinity and $P_{1}$ a polynomial, so that $f^{\prime} / f$ is rational at infinity, by $(6.1)$, contrary to assumption.

Assume henceforth that $d \neq 0$ in (6.1), which makes $g$ meromorphic of finite order in $\Omega\left(r_{1}\right)$. Suppose that $f$ has no poles in some $\Omega\left(r_{2}\right)$. Then $g$ has no zeros and poles there and $g=R_{2} e^{P_{2}}$ in (6.1), with $R_{2}$ rational at infinity and $P_{2}$ a polynomial. This gives, since $f^{\prime} / f$ is not rational at infinity,

$$
\frac{f^{\prime}}{f}=R+S e^{P}, \quad S P^{\prime} \not \equiv 0
$$

where $R$ and $S$ are rational at infinity and $P$ is a polynomial. It follows using [11, Lemma 3.5] that

$$
\begin{aligned}
& \frac{1}{g^{k}}=\frac{F}{f} \\
& =S^{k} e^{k P}+e^{(k-1) P}\left(k S^{k-1} R+a_{k-1} S^{k-1}+\frac{k(k-1)}{2} S^{k-2}\left(S^{\prime}+P^{\prime} S\right)\right)+\ldots
\end{aligned}
$$

Since $F / f$ has neither zeros nor poles in $\Omega\left(r_{2}\right)$, the coefficient of $e^{(k-1) P}$ must vanish identically, leading to the first equation of (1.5), with $H^{\prime}=S e^{P}$, and to $F / f=$ $S^{k} e^{k P}=\left(H^{\prime}\right)^{k}$. Here $H^{\prime \prime} / H^{\prime}$ does not vanish at infinity, because $P^{\prime}$ does not.

Suppose next that $f$ has an unbounded sequence of poles. At a pole $z$ of $f$, with $|z|$ large and with multiplicity $m$, equations (5.2) and (6.1) deliver

$$
\frac{1}{d^{k}}=\chi(m)=\frac{m(m+1) \ldots(m+k-1)}{(m+(k-1) / 2)^{k}},
$$

so that $d^{k}$ must be real and greater than 1 , by the arithmetic-geometric mean inequality. A further application of the same inequality to $\chi^{\prime} / \chi$ shows that all 
poles $z$ of $f$ with $|z|$ sufficiently large have fixed multiplicity $m$. Set $T_{1}=f^{\prime} / f$. Since $g^{k}$ and $T_{1}$ have finite order, standard estimates [8] give $M_{1}>0$ such that $T_{1}^{(j)}(z) / T_{1}(z)=O\left(|z|^{M_{1}}\right)$ and $g^{(j)}(z) / g(z)=O\left(|z|^{M_{1}}\right)$, for $|z|$ outside a set $F_{0}$ of finite measure and $1 \leq j \leq k$. If $|z| \notin F_{0}$ and $\log ^{+}\left|T_{1}(z)\right| / \log |z|$ is sufficiently large this leads, using (6.1) and [11, Lemma 3.5], to

$$
\frac{1}{g(z)^{k}}=\frac{F(z)}{f(z)}=T_{1}(z)^{k}+\ldots=(1+o(1)) T_{1}(z)^{k}=(1+o(1)) \frac{d^{k}}{g(z)^{k}},
$$

which is a contradiction since $d^{k}>1$. Thus $\log ^{+}\left|f^{\prime}(z) / f(z)\right|=O(\log |z|)$ for $|z|$ outside a set of finite measure and applying the Wiman-Valiron theory [12] to $1 / f$ shows that $f$ has finite order. Furthermore, since $f$ and $g$ have finite order and all poles $z$ of $f$ with $|z|$ sufficiently large have fixed multiplicity $m$, the function $G_{0}=f^{\prime} / f+m g^{\prime} / g$ is rational at infinity. Substituting $f^{\prime} / f=-m g^{\prime} / g+G_{0}$ into (6.1) produces a first order linear differential equation for $g$ of form

$$
g^{\prime}+\delta g=d_{0},
$$

with $d_{0} \in \mathbf{C}$ and $\delta$ rational at infinity, and with $\delta(\infty) \neq 0$, because $f / F=g^{k}$ has an essential singularity at infinity. This equation may be solved to give $g=d_{0} H / H^{\prime}$, where $H^{\prime \prime} / H^{\prime}=\delta$ and $H(z) \neq \infty$ and $H^{\prime}(z) \neq 0$ for large $z$ in a sector containing an unbounded sequence of poles of $f$. It follows, using (6.1) again, that

$$
\frac{g^{\prime}}{g}=\frac{H^{\prime}}{H}-\frac{H^{\prime \prime}}{H^{\prime}}, \quad \frac{f^{\prime}}{f}=-\frac{a_{k-1}}{k}-\left(\frac{k-1}{2}\right) \frac{H^{\prime \prime}}{H^{\prime}}+d_{1} \frac{H^{\prime}}{H}, \quad d_{1} \in \mathbf{C} .
$$

Now comparing residues shows that $d_{1}=-m$ in (6.4), giving the second equation of (1.5).

To determine the solutions of $L[y]=0$, write

$$
\phi=\left(H^{\prime}\right)^{-k} e^{H}, \quad \psi=\left(H^{\prime}\right)^{-k} H^{-m}, \quad \delta=\frac{H^{\prime \prime}}{H^{\prime}}, \quad M_{k}=(D+\delta) \cdots(D+k \delta), \quad D=\frac{d}{d z} .
$$

Then it is easy to verify that

$$
\Phi=M_{k}[\phi]=e^{H}, \quad \Psi=M_{k}[\psi]=c H^{-m-k}, \quad M_{k}\left[\left(H^{\prime}\right)^{-k} P_{k-1}(H)\right]=0,
$$

where $P_{k-1}$ denotes any polynomial of degree at most $k-1$. In fact, the action of the differential operator $M_{k}$ on $\phi, \psi$ and $\left(H^{\prime}\right)^{-k} P_{k-1}(H)$ amounts to $k$ times differentiating with respect to $H$ the terms $e^{H}, H^{-m}$ and $P_{k-1}(H)$. Define $Z$ locally by

$$
\frac{Z^{\prime}}{Z}=-\frac{a_{k-1}}{k}+\left(\frac{k+1}{2}\right) \frac{H^{\prime \prime}}{H^{\prime}}
$$

Then a standard change of variables gives $L_{k}=D^{k}+\ldots+\widetilde{A}_{1} D+\widetilde{A}_{0}$, with coefficients which are readily computable and rational at infinity, such that $L_{k}[Z y]=Z M_{k}[y]$, and the last equation of (6.5) shows that $L_{k}[w]=0$ has linearly independent solutions $y_{j}$ given locally by (1.6).

The next step is to show that $L_{k}=L$. When $f$ has no poles in some $\Omega\left(r_{2}\right)$, combining the first equation of (1.5) with (6.3) and the remarks immediately following it yields

$$
Z \phi=c f, \frac{L[f]}{f}=\frac{F}{f}=S^{k} e^{k P}=\left(H^{\prime}\right)^{k}=\frac{\Phi}{\phi}=\frac{M_{k}[\phi]}{\phi}=\frac{Z M_{k}[\phi]}{c f}=\frac{L_{k}[c f]}{c f}=\frac{L_{k}[f]}{f} .
$$

Thus the operators $L$ and $L_{k}$ must agree: otherwise $f$ satisfies a homogeneous linear differential equation with coefficients which are rational at infinity, and so has finite 
order, contradicting (6.2). On the other hand, when $f$ has an unbounded sequence of poles, (1.5) and (6.4) lead to

$$
Z \psi=c f, \quad \frac{L[f]}{f}=\frac{F}{f}=\frac{1}{g^{k}}=c\left(\frac{H^{\prime}}{H}\right)^{k}=\frac{c \Psi}{\psi}=\frac{c M_{k}[\psi]}{\psi}=\frac{c Z M_{k}[\psi]}{f}=\frac{c L_{k}[f]}{f} .
$$

Again the operators $L$ and $c L_{k}$ must agree, and $c$ must be 1 , because otherwise $f$ cannot have an unbounded sequence of poles. Thus, in both cases, the $y_{j}$ solve $L[y]=0$. Next, using (1.5) and (1.6) shows, after multiplying $y_{2}$ by a constant if necessary, that

$$
\frac{f^{\prime}}{f}=\frac{y_{1}^{\prime}}{y_{1}}+\left(\frac{y_{2}}{y_{1}}\right)^{\prime} \quad \text { or } \quad \frac{f^{\prime}}{f}=\frac{y_{1}^{\prime}}{y_{1}}-m\left(\frac{y_{2}^{\prime}}{y_{2}}-\frac{y_{1}^{\prime}}{y_{1}}\right)
$$

This gives $f=c y_{1} \exp \left(y_{2} / y_{1}\right)$ or $f=c y_{1}^{m+1} y_{2}^{-m}$ as asserted.

Finally, set $\widetilde{M}_{2}=(D+(k-1) \delta)(D+k \delta)$. There exists an operator $\widetilde{L}_{2}=D^{2}+$ $\widetilde{a}_{1} D+\widetilde{a}_{0}$, with coefficients which are rational at infinity, such that $\widetilde{L}_{2}[Z y]=Z \widetilde{M}_{2}[y]$ and

$$
\widetilde{L}_{2}[Z \phi]=Z \widetilde{M}_{2}[\phi]=Z\left(H^{\prime}\right)^{2-k} e^{H}, \quad \widetilde{L}_{2}[Z \psi]=Z \widetilde{M}_{2}[\psi]=c Z\left(H^{\prime}\right)^{2-k} H^{-m-2} .
$$

Since $f$ equals $c Z \phi$ or $c Z \psi$, there exists $r_{3}>0$ such that $\widetilde{L}_{2}[f]$ has no zeros in $\Omega\left(r_{3}\right)$, and Proposition 6.1 is proved.

\section{Annihilators}

The remainder of the proof of Theorem 1.3 focuses on the case where the operators $L$ and $M$ differ. In this case Lemma 5.3 ensures that if $\phi$ is a non-trivial solution of $L[y]=0$, and $\psi$ is a non-trivial solution of $N[y]=0$, then $\chi=\phi^{\prime} \psi+\phi V[\psi]$ solves $M[y]=0$. Here $\chi$ may vanish identically, in which case $\psi$ will be said to annihilate $\phi$, and vice versa. This notion makes sense when $\phi$ and $\psi$ are both analytic solutions, and also when they are both formal solutions. The terminology in this section is as in Section 4, and the convention that exponential parts have zero constant term still applies. The following variant of an auxiliary result from [2] is key to the proof of Theorem 1.3.

Lemma 7.1. [2] Assume that $L \neq M$ and take a canonical formal solution $G$ of $N[y]=0$ which is free of logarithms and has exponential part $\kappa$. In addition, take a fundamental set of canonical formal solutions $f_{1}, \ldots, f_{k}$ of $L[y]=0$, such that $f_{j}$ has exponential part $q_{j}$, and a fundamental set of canonical formal solutions $w_{1}, \ldots, w_{k}$ of $M[y]=0$, where $w_{j}$ has exponential part $s_{j}$. Then the following conclusions hold.

(i) Each $W_{j}=f_{j}^{\prime} G+f_{j} V[G]$ is either identically zero or a canonical formal solution of $M[y]=0$ with exponential part $q_{j}+\kappa$.

(ii) There exists $\lambda=\lambda(G) \in\{1, \ldots, k\}$ such that the collection $s_{1}, \ldots, s_{k}$ consists of

$$
q_{j}+\kappa \quad(j \neq \lambda), \quad q_{\lambda}-(k-1) \kappa .
$$

(iii) If the $W_{j}$ are linearly dependent, then $G$ annihilates a canonical formal solution $g_{1}$ of $L[y]=0$ with exponential part $q_{\lambda}$, and every formal solution of $L[y]=0$ which is annihilated by $G$ is a constant multiple of $g_{1}$.

(iv) If $\kappa$ is not identically zero, then the $W_{j}$ are linearly dependent. 
Proof. Conclusion (i) follows immediately from Lemma 5.3. Next, Lemma 4.1 and Abel's identity give, since $a_{k-1}(\infty)=A_{k-1}(\infty)=0$,

$$
\sum_{j=1}^{k} q_{j}=\sum_{j=1}^{k} s_{j}=0 .
$$
yield

Suppose first that the $W_{j}$ are linearly independent. Then (i), (7.2) and Lemma 4.1

$$
0=\sum_{j=1}^{k} s_{j}=\sum_{j=1}^{k}\left(q_{j}+\kappa\right)=k \kappa,
$$

which implies that $\kappa=0$ and that $\left\{s_{1}, \ldots, s_{k}\right\}=\left\{q_{1}, \ldots, q_{k}\right\}$, again by Lemma 4.1. This proves conclusion (iv), and that (7.1) applies when the $W_{j}$ are linearly independent.

Now suppose that the $W_{j}$ are linearly dependent: then $G$ annihilates a nontrivial solution $g_{1}$ of $L[y]=0$. It may be assumed that the exponential parts and formal series appearing in $G$ and the $f_{j}$ and $w_{j}$ all involve integer powers of $z^{1 / p}$, for some fixed $p \in \mathbf{N}$. Because $G$ is free of logarithms, (5.16) implies that $V[G] / G$ is a formal series in descending powers of $z^{1 / p}$, and therefore so is $g_{1}^{\prime} / g_{1}$. Thus $g_{1}$ is a canonical formal solution of $L[y]=0$, and by Lemma 4.1 it may be assumed that $g_{1}=f_{1}$; moreover, every formal solution $g_{2}$ of $L[y]=0$ which is annihilated by $G$ has $W\left(g_{1}, g_{2}\right)=0$, so that $g_{2}$ is a constant multiple of $g_{1}$. This proves (iii).

Now set $U_{j}=f_{j}^{\prime} G+f_{j} V[G]$. Then $U_{1} \equiv 0$, but $U_{2}, \ldots, U_{k}$ are linearly independent, and $M[y]=0$ has a fundamental set $\left\{U^{*}, U_{2}, \ldots, U_{k}\right\}$ of canonical formal solutions, with exponential parts $s^{*}, q_{2}+\kappa, \ldots, q_{k}+\kappa$ respectively. Using (7.2) twice, as well as Lemma 4.1, shows that these exponential parts have sum 0 and $s^{*}=q_{1}-(k-1) \kappa$, which leads to $(7.1)$.

The following lemma, in which transcendentally fast means faster than any power of $z$, gives a sufficient condition for an analytic solution of $N[y]=0$ to annihilate a solution of $L[y]=0$.

Lemma 7.2. Suppose that $L \neq M$. Then $g(z)$ cannot tend to 0 transcendentally fast as $z \rightarrow \infty$ in a sector, and the equation $N[y]=0$ cannot have a fundamental set of canonical formal solutions with the same exponential part. Moreover, if $G$ is a non-trivial analytic solution of $N[y]=0$ and $G(z)$ tends to 0 transcendentally fast as $z \rightarrow \infty$ in a sector $S$, then $G$ annihilates a non-trivial analytic solution of $L[y]=0$.

Proof. If $g$ tends to zero transcendentally fast on a sector, then $F / f=g^{-k}$ tends to infinity transcendentally fast there; since $f$ has finite order by Lemma 5.3, this contradicts standard estimates [8] for logarithmic derivatives $f^{(j)} / f$.

Next, if $N[y]=0$ has a fundamental set of canonical formal solutions with the same exponential part $\kappa$, then $\kappa$ is a polynomial in $z$, by Lemma 4.1 and Abel's identity. Here $\kappa$ cannot be the zero polynomial, because $g^{k}$ is transcendental, and so there exists a sector on which every solution of $N[y]=0$, including $g$, tends to zero transcendentally fast, which is a contradiction.

Assume now that $G$ is a non-trivial analytic solution of $N[y]=0$ which tends to 0 transcendentally fast in a sector $S$, but annihilates no non-trivial solution of $L[y]=0$. Then there exist $k$ solutions $f_{j}$ of $L[y]=0$ such that the $f_{j}^{\prime} G+f_{j} V[G]$ are linearly independent solutions of $M[y]=0$ on $S$. Because $N[y]=0$ has order at most 3 and at least two distinct exponential parts, the asymptotics in Section 4 
give rise to a subsector $S^{*}$ of $S$ on which $G(z) \neq 0$ and $G^{(j)}(z) / G(z)=O\left(|z|^{q}\right)$ as $z \rightarrow \infty$, for some $q \in \mathbf{N}$ and all $j \in\{1, \ldots, k\}$. This is clear if one solution $h_{j}$ as in (4.2) dominates the others on a subsector, and so evidently holds unless there are two solutions $h_{j}$ as in (4.2), with the same exponential part, for which the powers $\gamma_{j}$ differ by $\delta \in i \mathbf{R} \backslash\{0\}$; but in this case, for any given $A \in \mathbf{C}^{*}$, a subsector may be chosen on which $\log \left|z^{\delta}-A\right|$ is bounded. Define functions $Y, \phi$ and $\Phi$ on $S^{*}$ by

$$
\frac{Y^{\prime}}{Y}=-a_{k-1}=-A_{k-1}, \quad-\frac{\phi^{\prime}}{\phi}=\frac{V[G]}{G}=\alpha \frac{G^{\prime \prime}}{G}+\beta \frac{G^{\prime}}{G}+\gamma, \quad \Phi=L[\phi] .
$$

It follows that

$$
\begin{aligned}
c Y & =W\left(f_{1}^{\prime} G+f_{1} V[G], \ldots, f_{k}^{\prime} G+f_{k} V[G]\right) \\
& =W\left(f_{1}^{\prime} G-f_{1}\left(\phi^{\prime} / \phi\right) G, \ldots, f_{k}^{\prime} G-f_{k}\left(\phi^{\prime} / \phi\right) G\right)=(\phi G)^{k} W\left(\left(f_{1} / \phi\right)^{\prime}, \ldots,\left(f_{k} / \phi\right)^{\prime}\right) \\
& =(\phi G)^{k} W\left(1, f_{1} / \phi, \ldots, f_{k} / \phi\right)=\phi^{-1} G^{k} W\left(\phi, f_{1}, \ldots, f_{k}\right) .
\end{aligned}
$$

This delivers in turn

$$
\frac{\Phi}{\phi}=\frac{L[\phi]}{\phi}=\frac{c W\left(f_{1}, \ldots, f_{k}, \phi\right)}{Y \phi}=\frac{c}{G^{k}}
$$

so that $\Phi(z) / \phi(z)$ tends to infinity transcendentally fast in the sector $S^{*}$. But (7.3) implies that there exist $q^{\prime}, q^{\prime \prime} \in \mathbf{N}$ with $\phi^{\prime}(z) / \phi(z)=O\left(|z|^{q^{\prime}}\right)$ as $z \rightarrow \infty$ in $S^{*}$, and hence $\Phi(z) / \phi(z)=O\left(|z|^{q^{\prime \prime}}\right)$ as $z \rightarrow \infty$ on a subsector of $S^{*}$, a contradiction.

\section{The second special case}

Proposition 8.1. With the hypotheses of Theorem 1.3, suppose in addition that $L \neq M$ and that there exist $E \in \mathbf{N}$ and a function $R$ which is rational at infinity such that all poles $z$ of $\mathfrak{f}(z)=f\left(z^{E}\right)$ with $|z|$ sufficiently large have multiplicity $R(z)$. Then $f$ satisfies at least one of conclusions (i) and (ii) of Theorem 1.3.

The proof of Proposition 8.1 will occupy the remainder of this section. Observe first that $f$ has finite order and an unbounded sequence of poles, by Lemma 5.3. Next, it may be assumed that $E=1$. To see this, let $\omega=\exp (2 \pi i / E)$ and let $z_{0}$ be large and a pole of $f$ of multiplicity $m_{0}$. Let $w_{0}^{E}=z_{0}$. Then $w_{0}$ is a pole of $\mathfrak{f}$ of multiplicity $m_{0}=R\left(w_{0}\right)$. This is true for all $E$ choices of $w_{0}$ and so $R(z)=R(\omega z)$ for all large $z$, which gives $R(z)=S\left(z^{E}\right)$ for some function $S$ which is rational at infinity. Thus the multiplicity $m_{0}$ of the pole of $f$ at $z_{0}$ satisfies $m_{0}=R\left(w_{0}\right)=S\left(w_{0}^{E}\right)=S\left(z_{0}\right)$. Assume for the remainder of this section that $E=1$.

Lemma 8.1. There exist functions $d_{0}, d_{1}$, both rational at infinity, such that $f$ and $g$ satisfy

$$
\frac{f^{\prime}}{f}=d_{0} \frac{g^{\prime}}{g}+d_{1} .
$$

Moreover, $d_{0}$ either has $d_{0}(\infty)=\infty$ or is constant and equal to a negative integer.

Proof. Let $d_{0}=-R$. By the remark following (5.1), there exists $r_{0}>0$ such that $f^{\prime} / f-d_{0} g^{\prime} / g$ has no poles in $\Omega\left(r_{0}\right)$, and so is rational at infinity since $g^{k}$ and $f$ have finite order. The last assertion follows from the fact that $f$ has an unbounded sequence of poles.

Lemma 8.2. There exist functions $\nu_{1}, \nu_{0}$, both rational at infinity, such that $g$ satisfies (1.8). 
Proof. The equation (8.1) yields, using (5.1),

$$
-h=\left(\frac{f^{\prime}}{f}\right) g=d_{0} g^{\prime}+d_{1} g, \quad-h^{\prime}=d_{0} g^{\prime \prime}+\left(d_{0}^{\prime}+d_{1}\right) g^{\prime}+d_{1}^{\prime} g .
$$

Combining this with (5.15) gives

$$
0=\left(d_{0}-\frac{k-1}{2}\right) g^{\prime \prime}+\left(d_{0}^{\prime}+d_{1}+\frac{a_{k-1}}{k}\right) g^{\prime}+\left(d_{1}^{\prime}+\frac{a_{k-1}^{\prime}+c_{k-2}-C_{k-2}}{k}\right) g
$$

and an equation (1.8), as asserted, since $d_{0}-(k-1) / 2 \not \equiv 0$ by Lemma 8.1.

From (1.8), (5.16) and (8.1) it follows that

$$
-d_{0} g^{\prime}-d_{1} g=-\left(\frac{f^{\prime}}{f}\right) g=h=V[g]=\left(\beta-\alpha \nu_{1}\right) g^{\prime}+\left(\gamma-\alpha \nu_{0}\right) g
$$

and so, since $g^{k}$ has an unbounded sequence of zeros,

$$
-d_{0}=\beta-\alpha \nu_{1}, \quad-d_{1}=\gamma-\alpha \nu_{0} .
$$

In the next lemma the convention that exponential parts have zero constant term is retained.

Lemma 8.3. There exists an equation (4.3), with $A^{*}$ rational at infinity, such that $y U^{-1 / 2}$ solves (4.3) for every solution $y$ of (1.8), where $U^{\prime} / U=-\nu_{1}$. The equation (1.8) has a pair of linearly independent canonical formal solutions with distinct exponential parts, and (4.3) has an irregular singular point at infinity. If $\kappa$ is a non-zero exponential part for equation (1.8), then there exists a locally analytic solution $u_{1}$ of (1.8), with exponential part $\kappa$, which continues without zeros in some $\Omega\left(r_{2}\right)$ and annihilates a non-trivial locally analytic solution $y_{1}$ of $L[y]=0$, where $y_{1}$ is given by

$$
\frac{y_{1}^{\prime}}{y_{1}}=d_{0} \frac{u_{1}^{\prime}}{u_{1}}+d_{1}
$$

Moreover, both $z u_{1}^{\prime}\left(z^{2}\right) / u_{1}\left(z^{2}\right)$ and $z y_{1}^{\prime}\left(z^{2}\right) / y_{1}\left(z^{2}\right)$ are rational at infinity.

Proof. The existence of the equation (4.3) solved by $y U^{-1 / 2}$ for every solution $y$ of (1.8) is a standard consequence of Abel's identity. Now the exponential parts $\kappa_{1}, \kappa_{2}$ for (1.8) are polynomials in $z^{1 / 2}$, by (4.4), and their sum is a polynomial in $z$; thus $\kappa_{j}(z)=Q_{j}(z)+z^{1 / 2}(-1)^{j} Q^{*}(z)$ with $Q^{*}$ and the $Q_{j}$ polynomials in $z$.

Suppose that $\kappa_{1}=\kappa_{2}=\kappa_{0}$. Then $\kappa_{0}$ is a polynomial, and must be non-constant since $g$ satisfies (1.8) and $f / F=g^{k}$ has an essential singularity at infinity. But this implies the existence of a sector on which every solution of (1.8), including $g$, tends to zero transcendentally fast as $z \rightarrow \infty$, which contradicts Lemma 7.2. Thus $\kappa_{1} \neq \kappa_{2}$, so that (4.3) has an irregular singular point at infinity, and at least one canonical formal solution of (1.8) has non-zero exponential part.

Take a canonical formal solution $u_{1}$ of (1.8) with exponential part $\kappa \neq 0$. Then $u_{1}$ is given by a formal expression as in (4.1), but free of logarithms, and $u_{1}^{\prime} / u_{1}$ is a formal series in descending powers of $z^{1 / 2}$. Since $f$ has finite order and an unbounded sequence of poles, the function $g^{\prime} / g$ is not rational at infinity. Thus $g$ cannot solve a first order homogeneous linear differential equation with coefficients which are rational at infinity, and so the division algorithm for linear differential operators $[15$, p.126] shows that the operator $N$ of Lemma 5.3 must satisfy $N=$ $N_{0} \circ\left(D^{2}+\nu_{1} D+\nu_{0}\right)$, for some operator $N_{0}$ of order 1 or 0 . Hence every solution of (1.8), including $u_{1}$, solves $N[y]=0$. It follows from Lemma 7.1 that $u_{1}$ annihilates 
some canonical formal solution $y_{1}$ of $L[y]=0$. This gives, using (1.8), (5.16) and (8.3),

$$
-y_{1}^{\prime} u_{1}=y_{1} V\left[u_{1}\right]=y_{1}\left(\left(\beta-\alpha \nu_{1}\right) u_{1}^{\prime}+\left(\gamma-\alpha \nu_{0}\right) u_{1}\right)=y_{1}\left(-d_{0} u_{1}^{\prime}-d_{1} u_{1}\right),
$$

and hence (8.4). Thus $y_{1}^{\prime} / y_{1}$ is also a formal series in $z^{1 / 2}$, and the hypotheses of Lemma 3.3 are satisfied with $p=y_{1}$ and $q=u_{1}$. Hence (3.2) holds with the $b_{j}$ rational at infinity and $b_{k} \not \equiv 0$ by Lemma 8.1. But $L\left[y_{1}\right]=0$, and so $u_{1}^{\prime} / u_{1}$ is algebraic at infinity, that is, $u_{1}^{\prime} / u_{1}$ solves a polynomial equation with coefficients which are rational at infinity. In particular, the series for $u_{1}^{\prime} / u_{1}$ converges for large $z$ in some sector, as does that for $y_{1}^{\prime} / y_{1}$, by (8.4), and $u_{1}$ and $y_{1}$ are analytic local solutions of (1.8) and $L[y]=0$ respectively. Since the algebraic equation for $u_{1}^{\prime} / u_{1}$ has only finitely many branches for its solutions, and each branch has no poles in some sector $|z|>r_{2},|\arg z|<4 \pi$, it follows that $u_{1}$ continues without zeros in $\Omega\left(r_{2}\right)$. This means that, as $z$ crosses a critical ray of (4.3), the solution $u_{1} U^{-1 / 2}$ of (4.3) must change from small to large or vice versa. Therefore continuing twice around a circle $|z|=r_{3}>r_{2}$ brings $u_{1} U^{-1 / 2}$ back to a constant multiple of itself, and the same is true for $u_{1}$. Thus $z u_{1}^{\prime}\left(z^{2}\right) / u_{1}\left(z^{2}\right)$ is rational at infinity, and so is $z y_{1}^{\prime}\left(z^{2}\right) / y_{1}\left(z^{2}\right)$ by (8.4).

Choose a critical ray $\arg z=\theta^{*}$ for the equation (4.3) and a sector $S^{*}$, symmetric about the critical ray, and with internal angle slightly less than $4 \pi /\left(2+\operatorname{deg}_{\infty} A^{*}\right)$, in which $f$ has an unbounded sequence of poles, these being zeros of $g$. In the sector $S^{*}$, equation (4.3) has two linearly independent zero-free analytic solutions, by (4.4). Denote these by $u^{*}=u U^{-1 / 2}$ and $v^{*}=v U^{-1 / 2}$ say, where $u$ and $v$ solve (1.8). Here $u$ and $v$ have distinct exponential parts $\kappa_{u}$ and $\kappa_{v}$, each a polynomial in $z^{1 / 2}$, and it may be assumed that $\kappa_{u}$ is non-constant and

$$
\liminf _{z \rightarrow \infty, z \in S^{*}}\left|\frac{\kappa_{v}(z)}{\kappa_{u}(z)}\right| \leq 1, \quad g=v-u
$$

since $u$ and $v$ may be interchanged and multiplied by constants. Now Lemma 8.3 shows that there exist locally analytic solutions $u_{1}$ of (1.8) and $y_{1}$ of $L[y]=0$ respectively, such that $u_{1}$ has exponential part $\kappa_{u}$, while (8.4) holds and both $z u_{1}^{\prime}\left(z^{2}\right) / u_{1}\left(z^{2}\right)$ and $z y_{1}^{\prime}\left(z^{2}\right) / y_{1}\left(z^{2}\right)$ are rational at infinity. Thus $u_{1}$ must be a constant multiple of $u$ and so, by (8.1),

$$
T_{1}=\frac{y_{1}^{\prime}}{y_{1}}=d_{0} \frac{u^{\prime}}{u}+d_{1}, \quad \frac{f^{\prime}}{f}=d_{0} \frac{g^{\prime}}{g}+d_{1}=d_{0}\left(\frac{g^{\prime}}{g}-\frac{u^{\prime}}{u}\right)+\frac{y_{1}^{\prime}}{y_{1}} .
$$

Poles $z$ of $f$ occur where $v(z) / u(z)=1$, and have multiplicity equal to $-d_{0}(z)$, by (8.6). Furthermore, by $(4.4), \zeta=(1 / 2 \pi i) \log \left(v^{*} / u^{*}\right)=(1 / 2 \pi i) \log (v / u)$ maps $S^{*}$ conformally onto a domain containing a right or left half-plane $\pm \operatorname{Re} \zeta>M_{0}>0$. Since $d_{0}$ takes integer values at all points in $S^{*}$ where $\zeta$ is integer-valued, applying Lemma 3.1 shows that there exists a polynomial $Q$ such that

$$
d_{0}=Q(T), \quad T=2 \pi i \zeta=\log \left(\frac{v}{u}\right) .
$$

The second equation of (8.6) can now be written in the form

$$
\begin{aligned}
\frac{f^{\prime}}{f} & =Q(T)\left(\frac{v^{\prime}-u^{\prime}}{v-u}-\frac{u^{\prime}}{u}\right)+\frac{y_{1}^{\prime}}{y_{1}}=Q(T) \frac{v^{\prime} u-u^{\prime} v}{(v-u) u}+\frac{y_{1}^{\prime}}{y_{1}} \\
& =Q(T) \frac{v^{\prime} / v-u^{\prime} / u}{1-u / v}+\frac{y_{1}^{\prime}}{y_{1}}=\frac{Q(T) T^{\prime}}{1-e^{-T}}+\frac{y_{1}^{\prime}}{y_{1}}=\frac{Q(T) T^{\prime}}{1-e^{-T}}+T_{1},
\end{aligned}
$$


which gives (1.7), and it suffices to consider two cases.

8.1. Case I. Suppose first that $Q$ is constant and one exponential part for (1.8) is 0 . Then $d_{0}=Q(T)$ is constant and $v$ has exponential part 0 in $S^{*}$, because $u$ does not. A pole of $f$ of multiplicity $m_{0}$ in $S^{*}$ gives $v / u=1$ and $g^{\prime}=v^{\prime}-u^{\prime}=v^{\prime}-\left(u^{\prime} / u\right) v=T^{\prime} v$, as well as (5.2). Since all poles of $f$ in $S^{*}$ with $|z|$ sufficiently large have fixed multiplicity $-d_{0}$, it follows from Lemma 3.1 and $(5.2)$ that $\left(T^{\prime} v\right)^{-k}$, which also has exponential part 0 in $S^{*}$, must be constant, and so must $v^{\prime}-\left(u^{\prime} / u\right) v$. But then $W(u, v) / u$ is constant, and so $\nu_{1}=-u^{\prime} / u$ in (1.8). Because $u$ solves (1.8), it must be the case that $\nu_{0}=-\left(u^{\prime} / u\right)^{\prime}=\nu_{1}^{\prime}$. Now comparing (1.8) and (8.2) shows that, since $d_{0}$ is constant, $c_{k-2}-C_{k-2}$ must vanish, so that Proposition 6.1 may be applied, and $f$ satisfies conclusion (i) of Theorem 1.3.

8.2. Case II. Assume now that either both exponential parts for (1.8) are non-zero, or $Q$ is non-constant.

Lemma 8.4. The solution $v$ continues without zeros in some $\Omega\left(r_{2}\right)$, and $z v^{\prime}\left(z^{2}\right) /$ $v\left(z^{2}\right)$ is rational at infinity.

Proof. Suppose first that both exponential parts for (1.8) are non-zero. Then Lemma 8.3 gives a solution $V_{1}$ of (1.8), such that $V_{1}$ and $u$ are linearly independent and $V_{1}$ continues without zeros in some $\Omega\left(r_{2}\right)$, with $z V_{1}^{\prime}\left(z^{2}\right) / V_{1}\left(z^{2}\right)$ rational at infinity. Since $u$ and $v$ are linearly independent and zero-free in $S^{*}$, the solution $V_{1}$ must be a constant multiple of $v$.

Now suppose that $v$ has exponential part 0 in $S^{*}$ : then $Q$ is non-constant, and (8.7) implies that $T=\log (v / u)$ is algebraic at infinity. Thus $v$ continues without zeros in some $\Omega\left(r_{2}\right)$, because $u$ does, and the same argument as applied to $u$ in the proof of Lemma 8.3 shows that $z v^{\prime}\left(z^{2}\right) / v\left(z^{2}\right)$ is rational at infinity as asserted.

The functions $u^{\prime} / u, v^{\prime} / v$ and $T^{\prime}=v^{\prime} / v-u^{\prime} / u$ are all defined for large $z \in S^{*}$ and given by convergent series in descending powers of $z^{1 / 2}$. Denote by $\widehat{\psi}$ the result of continuing a function element $\psi$ once counter-clockwise around a circle $|z|=r_{3}>r_{2}$, starting in $S^{*}$. Since $u$ and $v$ both continue without zeros, there exists $\zeta_{0} \in \mathbf{C}$ such that

$$
\begin{array}{lll}
\text { (a) } \widehat{u}=c u, & \widehat{v}=c v, & \widehat{T}=T+\zeta_{0} \quad \text { or } \\
\text { (b) } \widehat{u}=c v, & \widehat{v}=c u, & \widehat{T}=-T-\zeta_{0} .
\end{array}
$$

Lemma 8.5. There exist $d_{2} \in[0,1 / 2]$ and functions $E_{0}, E_{1}$ and $E_{2} \not \equiv 0$, each rational at infinity, such that

$$
\frac{u^{\prime}(z)}{u(z)}=\left(d_{2}-1\right) T^{\prime}(z)+o\left(\left|T^{\prime}(z)\right|\right), \quad \frac{v^{\prime}(z)}{v(z)}=d_{2} T^{\prime}(z)+o\left(\left|T^{\prime}(z)\right|\right)
$$

as $z \rightarrow \infty$ in $S^{*}$, while $E_{2} f^{\prime \prime}+E_{1} f^{\prime}+E_{0} f$ has no zeros in some $\Omega\left(r_{3}\right)$. If subcase (a) applies in (8.9), then $T^{\prime}$ is rational at infinity, with $T^{\prime}(\infty) \neq 0$, while if subcase (b) applies then $d_{2}=1 / 2$ and $H_{0}(z)=z^{1 / 2} T^{\prime}(z)$ is rational at infinity, with $H_{0}(\infty) \neq 0$.

Proof. Suppose first that subcase (a) applies in (8.9). Then $u^{\prime} / u, v^{\prime} / v$ and $T^{\prime}$ are all rational at infinity, and so is $T_{1}$ in (8.6). Thus applying Lemma 3.3 to $f$ and $g$ gives, in view of (8.1), (8.8) and Lemma 8.2, functions $E_{0}, E_{1}$ and $E_{2}$, each rational at infinity, such that $E_{2} \not \equiv 0$ and

$$
E_{2} \frac{f^{\prime \prime}}{f}+E_{1} \frac{f^{\prime}}{f}+E_{0}=\left(\frac{f^{\prime}}{f}-T_{1}\right)^{2}=\left(\frac{Q(T) T^{\prime}}{1-e^{-T}}\right)^{2} .
$$


Hence $E_{2} f^{\prime \prime}+E_{1} f^{\prime}+E_{0} f$ has no zeros in some $\Omega\left(r_{3}\right)$.

To prove the existence of $d_{2}$ in subcase (a), suppose first that $\operatorname{deg}_{\infty}\left(u^{\prime} / u\right)>$ $\operatorname{deg}_{\infty} T^{\prime}$. Then, as $z \rightarrow \infty$, with $\arg z$ arbitrary,

$$
\frac{v^{\prime}(z)}{v(z)}=\frac{u^{\prime}(z)}{u(z)}+T^{\prime}(z)=(1+o(1)) \frac{u^{\prime}(z)}{u(z)} .
$$

Since $u$ has non-zero exponential part, this gives a sector on which $u$ and $v$ both tend to zero transcendentally fast, and hence so does every solution of (1.8), including $g$, contradicting Lemma 7.2. Thus there exists $d_{2} \in \mathbf{C}$ such that (8.10) holds as $z \rightarrow \infty$, with $\arg z$ arbitrary, and $T^{\prime}(\infty) \neq 0$, since $u$ has non-zero exponential part. If $d_{2} \notin \mathbf{R}$, or if $d_{2} \in \mathbf{R} \backslash[0,1]$, then again there exists a sector on which $u, v$ and $g$ all tend to zero transcendentally fast, contradicting Lemma 7.2. Finally, (8.5) gives $d_{2} \leq 1 / 2$.

Assume now that subcase (b) holds in (8.9). Because $f$ has an unbounded sequence of poles in $S^{*}$ and $y_{1}$ continues without zeros, (8.8) leads to

$$
\widehat{T}^{\prime}=-T^{\prime}, \quad \frac{f^{\prime}}{f}=\frac{-Q(T) T^{\prime}}{1-e^{T+\zeta_{0}}}+\widehat{T}_{1}, \quad e^{\zeta_{0}}=1, \quad Q(T) T^{\prime}=\widehat{T}_{1}-T_{1}=T_{2}-T_{1} .
$$

Furthermore, $u^{\prime} / u+v^{\prime} / v=2 H_{1}$ and $u^{\prime} v^{\prime} / u v$ are rational at infinity, and so are $T_{1}+T_{2}$ and $T_{1} T_{2}$ by continuation of the first equation of (8.6). On the other hand (8.11) implies that $T^{\prime}(z)=2 z^{1 / 2} H_{2}(z)$, with $H_{2}$ rational at infinity. This yields

$$
\frac{u^{\prime}(z)}{u(z)}=H_{1}(z)-z^{1 / 2} H_{2}(z), \quad \frac{v^{\prime}(z)}{v(z)}=H_{1}(z)+z^{1 / 2} H_{2}(z) .
$$

Since $u$ has non-zero exponential part, either $\operatorname{deg}_{\infty} H_{1} \geq 0$ or $\operatorname{deg}_{\infty} H_{2} \geq-1$. Moreover, $\operatorname{deg}_{\infty} H_{2} \geq \operatorname{deg}_{\infty} H_{1}$ (and so $\operatorname{deg}_{\infty} H_{2} \geq-1$ ) in (8.12); otherwise there again exists a sector on which every solution of (1.8), including $g$, tends to zero transcendentally fast, contradicting Lemma 7.2. Thus (8.10) holds with $d_{2}=1 / 2$.

Applying Lemma 3.3 to $f$ and $g$ now gives, in view of Lemma 8.2 and (8.1), (8.8) and (8.11), functions $E_{0}, E_{1}$ and $E_{2}$, each rational at infinity, such that $E_{2} \not \equiv 0$ and

$$
E_{2} \frac{f^{\prime \prime}}{f}+E_{1} \frac{f^{\prime}}{f}+E_{0}=\left(\frac{f^{\prime}}{f}-T_{1}\right)\left(\frac{f^{\prime}}{f}-T_{2}\right)=-\frac{\left(Q(T) T^{\prime}\right)^{2}}{\left(1-e^{T}\right)\left(1-e^{-T}\right)},
$$

and so $E_{2} f^{\prime \prime}+E_{1} f^{\prime}+E_{0} f$ again has no zeros in some $\Omega\left(r_{3}\right)$.

Recall that $\zeta(z)=T(z) / 2 \pi i$ maps a subdomain of $S^{*}$ conformally onto a right or left half-plane. If $z_{1} \in S^{*}$ and $\zeta\left(z_{1}\right) \in \mathbf{Z}$ then $e^{T\left(z_{1}\right)}=v\left(z_{1}\right) / u\left(z_{1}\right)=1$, while $f$ has a pole at $z_{1}$ of multiplicity $-d_{0}\left(z_{1}\right)=-Q\left(T\left(z_{1}\right)\right)$, by (8.8), and (5.2) gives

$$
\begin{aligned}
\left(T^{\prime}\left(z_{1}\right) v\left(z_{1}\right)\right)^{-k} & =\left(v^{\prime}\left(z_{1}\right)-u^{\prime}\left(z_{1}\right)\right)^{-k}=g^{\prime}\left(z_{1}\right)^{-k}=Q_{0}\left(T\left(z_{1}\right)\right), \\
Q_{0} & =Q(Q-1) \ldots(Q-k+1) .
\end{aligned}
$$

Lemma 8.5 makes it possible to write, on $S^{*}$,

$$
T^{\prime}(z)^{k} v(z)^{k} Q_{0}(T(z))=e^{k d_{2} T(z)} u_{0}(z)=e^{2 \pi i k d_{2} \zeta(z)} u_{0}(z),
$$

in which $\log ^{+}\left|u_{0}(z)\right|=o(|T(z)|)=o(|\zeta(z)|)$ as $z \rightarrow \infty$ in $S^{*}$. Thus (8.13), (8.14) and Lemma 3.1 together imply that $k d_{2} \in \mathbf{Z}$ and $u_{0} \equiv 1$, so that $v$ and $u$ have representations, for some branch of $Q_{0}(T)^{1 / k}$,

$$
v=\frac{e^{d_{2} T}}{Q_{0}(T)^{1 / k} T^{\prime}}, \quad u=v e^{-T}=\frac{e^{\left(d_{2}-1\right) T}}{Q_{0}(T)^{1 / k} T^{\prime}}, \quad d_{2} \in[0,1 / 2], \quad k d_{2} \in \mathbf{Z},
$$


and if $T^{\prime}$ is not rational at infinity then $d_{2}=1 / 2$ and $k$ is even. Now Abel's identity, (1.8), (8.2), (8.6), (8.7) and (8.15) lead to

$$
\begin{aligned}
W_{0}= & W(u, v)=c e^{\left(2 d_{2}-1\right) T} Q_{0}(T)^{-2 / k}\left(T^{\prime}\right)^{-1}, \\
\nu_{1}= & \frac{d_{0}^{\prime}+d_{1}+a_{k-1} / k}{d_{0}-(k-1) / 2}=-\frac{W_{0}^{\prime}}{W_{0}}=\left(1-2 d_{2}\right) T^{\prime}+\frac{2 Q_{0}^{\prime}(T) T^{\prime}}{k Q_{0}(T)}+\frac{T^{\prime \prime}}{T^{\prime}}, \\
\frac{y_{1}^{\prime}}{y_{1}}= & Q(T)\left(\left(d_{2}-1\right) T^{\prime}-\frac{Q_{0}^{\prime}(T) T^{\prime}}{k Q_{0}(T)}-\frac{T^{\prime \prime}}{T^{\prime}}\right) \\
& +\left(Q(T)-\frac{k-1}{2}\right)\left(\left(1-2 d_{2}\right) T^{\prime}+\frac{2 Q_{0}^{\prime}(T) T^{\prime}}{k Q_{0}(T)}+\frac{T^{\prime \prime}}{T^{\prime}}\right)-Q^{\prime}(T) T^{\prime}-\frac{a_{k-1}}{k} .
\end{aligned}
$$

Hence $T_{3}=y_{1}^{\prime} / y_{1}+a_{k-1} / k$ is given by

$$
\begin{aligned}
T_{3}= & \frac{1}{k} \sum_{j=0}^{k-2}\left(\frac{j-k+1}{Q(T)-j}\right) Q^{\prime}(T) T^{\prime}-\left(d_{2}(Q(T)-k+1)+\frac{k-1}{2}\right) T^{\prime} \\
& -\left(\frac{k-1}{2}\right) \frac{T^{\prime \prime}}{T^{\prime}} .
\end{aligned}
$$

Thus $T_{1}=y_{1}^{\prime} / y_{1}$ belongs to the field $\widetilde{\Lambda}$ generated by $d_{0}=Q(T), T^{\prime}, a_{k-1}$ and their derivatives. Since $L\left[y_{1}\right]=0$, a standard change of variables gives a linear differential operator $\widetilde{L}$ with coefficients $\widetilde{c}_{j} \in \widetilde{\Lambda}$ such that

$$
L\left[y_{1} w\right]=y_{1} \widetilde{L}[w], \quad \widetilde{L}=\sum_{j=1}^{k} \widetilde{c}_{j} D^{j}, \quad \widetilde{c}_{k}=1, \quad D=\frac{d}{d z} .
$$

As $T_{1}$ is known, the $\widetilde{c}_{j}$ can be computed from the $a_{j}$, and vice versa. Using (8.5) and (8.8), write

$$
\begin{aligned}
& f=y_{1} \phi, \quad \frac{1}{(v-u)^{k}}=\frac{1}{g^{k}}=\frac{L[f]}{f}=\frac{\widetilde{L}[\phi]}{\phi}=\sum_{j=1}^{k} \widetilde{c}_{j} \frac{\phi^{(j)}}{\phi} \\
& \frac{\phi^{\prime}}{\phi}=\frac{S_{1}}{Y_{1}}, \quad S_{1}=R_{1,0}=Q(T) T^{\prime}, \quad Y_{1}=1-e^{-T}, \quad Y_{1}^{\prime}=T^{\prime}\left(1-Y_{1}\right) .
\end{aligned}
$$

There exist computable coefficients $R_{j, \mu} \in \widetilde{\Lambda}$ such that, for $j \in \mathbf{N}$,

$$
\begin{aligned}
\frac{\phi^{(j)}}{\phi} & =\frac{S_{j}}{Y_{1}^{j}}, \quad S_{j}=\sum_{\mu=0}^{j-1} R_{j, \mu} Y_{1}^{\mu}, \\
R_{j, 0} & =Q(T)(Q(T)-1) \ldots(Q(T)-j+1)\left(T^{\prime}\right)^{j} .
\end{aligned}
$$

The relations (8.18) hold by a straightforward induction argument, since the $S_{j}$ satisfy $S_{j+1}=Y_{1} S_{j}^{\prime}-j Y_{1}^{\prime} S_{j}+S_{1} S_{j}=Y_{1} S_{j}^{\prime}+j T^{\prime}\left(Y_{1}-1\right) S_{j}+S_{1} S_{j}, \quad R_{j+1,0}=(Q(T)-j) T^{\prime} R_{j, 0}$. Using (8.15), (8.17) and (8.18) now delivers

$$
\begin{aligned}
Q_{0}(T)\left(T^{\prime}\right)^{k}\left(1-Y_{1}\right)^{k d_{2}} & =Q_{0}(T)\left(T^{\prime}\right)^{k} e^{-k d_{2} T}=v^{-k}=\frac{Y_{1}^{k}}{(v-u)^{k}}=\sum_{j=1}^{k} \widetilde{c}_{j} S_{j} Y_{1}^{k-j} \\
& =\sum_{j=1}^{k} \sum_{\mu=0}^{j-1} \widetilde{c}_{j} R_{j, \mu} Y_{1}^{k-j+\mu}=\sum_{\mu=0}^{k-1} \sum_{j=\mu+1}^{k} \widetilde{c}_{j} R_{j, \mu} Y_{1}^{k-j+\mu}
\end{aligned}
$$




$$
=\sum_{\mu=0}^{k-1} \sum_{\nu=\mu}^{k-1} \widetilde{c}_{k+\mu-\nu} R_{k+\mu-\nu, \mu} Y_{1}^{\nu}=\sum_{\nu=0}^{k-1} Y_{1}^{\nu} \sum_{\mu=0}^{\nu} \widetilde{c}_{k+\mu-\nu} R_{k+\mu-\nu, \mu},
$$

in which $\nu=k-j+\mu$. Now $e^{-T}=u / v$ grows transcendentally fast on a subsector of $S^{*}$, whereas each element of the field $\widetilde{\Lambda}$ has form $\widetilde{v}(z)=v_{1}(z)+z^{1 / 2} v_{2}(z)$, with $v_{1}$ and $v_{2}$ rational at infinity. Thus $e^{-T}$ is transcendental over $\widetilde{\Lambda}$ and so is $Y_{1}=1-e^{-T}$. Since (8.7), (8.13), (8.18) and Lemma 8.1 together imply that $R_{j, 0} \not \equiv 0$ for $j=1, \ldots, k$ and that $\widetilde{c}_{k} R_{k, 0}=Q_{0}(T)\left(T^{\prime}\right)^{k}$, comparing the coefficients of $Y_{1}^{\nu}$, starting from $\nu=1$, determines successively $\widetilde{c}_{k-1}, \ldots, \widetilde{c}_{1}$ and hence $\left\{a_{0}, \ldots, a_{k-2}\right\}$.

The proof of Proposition 8.1 is complete, but it is worth remarking that (2.1), (2.2) and (2.3) show that $d_{2}=0$ and $d_{2}=1 / 3$ are both possible when $k=3$. Furthermore, Propositions 6.1 and 8.1 each give a solution $y_{0}$ of $L[y]=0$ on $S^{*}$, of the form (4.2), whose exponential part is a non-constant polynomial in $z^{1 / 2}$, and in $z$ if $k$ is odd. If $d_{2}=0$ or $d_{2}=1 / 2$ in (8.16), or if $f$ is given by (1.5), then $y_{0}=y_{1}$, by (8.7), Lemma 8.5 and the fact that $H^{\prime \prime} / H^{\prime}$ does not vanish at infinity in (1.5). On the other hand, if $0<d_{2}<1 / 2$ then $u$ and $v$ both have non-constant exponential part, by Lemma 8.5 and (8.15), and Lemma 7.2 gives a non-trivial solution $y_{2}$ of $L[y]=0$ annihilated by $v$; thus $y_{2}^{\prime} / y_{2}-y_{1}^{\prime} / y_{1}=d_{0}\left(v^{\prime} / v-u^{\prime} / u\right)=Q(T) T^{\prime}$, by (8.3), (8.6) and (8.7), and $y_{0} \in\left\{y_{1}, y_{2}\right\}$. It follows that $y_{0}^{\prime}(z) / y_{0}(z)=(1+o(1)) c z^{\lambda_{0}}$ and $y_{0}^{(j)}(z) / y_{0}^{(k)}(z)=(1+o(1)) c z^{(j-k) \lambda_{0}}$ as $z \rightarrow \infty$ in a subsector of $S^{*}$, for $j=0, \ldots, k-1$, where $\lambda_{0} \geq-1 / 2$, and $\lambda_{0} \geq 0$ if $k$ is odd. This implies that at least one $a_{j}$ has $a_{j}(z) \neq o\left(|z|^{(k-j) \lambda_{0}}\right)$ as $z \rightarrow \infty$, which is sharp by [17, (1.8)].

\section{A change of variables}

In order to prove Theorem 1.3 it now suffices, in view of Proposition 6.1, to show that the hypotheses of Proposition 8.1 are satisfied when $L \neq M$. Since the value of $E$ is immaterial in Proposition 8.1, a change of variables $z \rightarrow z^{n}$ may now be employed to ensure that, in the terminology of Section 4 , the integer $p$ is 1 , so that the exponential parts and associated asymptotic or formal series involve only integer powers. Indeed, let $k \geq 3$ and $n \geq 2$ be integers and let $f, F$ and $\mathfrak{f}$ satisfy

$$
F(z)=L[f](z)=f^{(k)}(z)+a_{k-1}(z) f^{(k-1)}(z)+\ldots+a_{0}(z) f(z), \quad \mathfrak{f}(z)=f\left(z^{n}\right),
$$

where the $a_{j}$ are rational at infinity with $a_{k-1}(\infty)=0$. Take linearly independent locally analytic solutions $f_{1}, \ldots, f_{k}$ of $L[y]=0$.

Lemma 9.1. For each integer $m \geq 1$ there exist rational functions $c_{p, m}(z)$, depending only on $m$ and $n$, such that

$$
f^{(m)}\left(z^{n}\right)=\sum_{p=1}^{m} c_{p, m}(z) \mathfrak{f}^{(p)}(z), \quad c_{m, m}(z)=\left(n z^{n-1}\right)^{-m} .
$$

Moreover, if $m \geq 2$ then $c_{m-1, m}(z) / c_{m, m}(z) \rightarrow 0$ as $z \rightarrow \infty$.

Proof. Clearly $\mathfrak{f}(z)=f\left(z^{n}\right)$ gives, as $z \rightarrow \infty$,

$f^{\prime}\left(z^{n}\right)=\left(n z^{n-1}\right)^{-1} \mathfrak{f}^{\prime}(z), \quad f^{\prime \prime}\left(z^{n}\right)=\left(n z^{n-1}\right)^{-2} \mathfrak{f}^{\prime \prime}(z)+c_{1,2}(z) \mathfrak{f}^{\prime}(z), \quad c_{1,2}(z)=O\left(|z|^{1-2 n}\right)$. 
Next, if the assertions of the lemma hold for some $m \geq 2$ then, as $z \rightarrow \infty$,

$$
\begin{aligned}
n z^{n-1} f^{(m+1)}\left(z^{n}\right) & =c_{m, m}(z) \mathfrak{f}^{(m+1)}(z)+\mathfrak{f}^{(m)}(z)\left(c_{m, m}^{\prime}(z)+c_{m-1, m}(z)\right)+\ldots \\
& =c_{m, m}(z)\left[\mathfrak{f}^{(m+1)}(z)+\mathfrak{f}^{(m)}(z) O(1 /|z|)+\ldots\right] .
\end{aligned}
$$

Now (9.1) and (9.2) yield, as $z \rightarrow \infty$,

$$
\begin{aligned}
F\left(z^{n}\right)= & \left(n z^{n-1}\right)^{-k}\left[\mathfrak{f}^{(k)}(z)+\mathfrak{f}^{(k-1)}(z) O(1 /|z|)+\ldots\right] \\
& +O\left(|z|^{-n}\right)\left(n z^{n-1}\right)^{1-k}\left[\mathfrak{f}^{(k-1)}(z)+\ldots\right]+\ldots \\
= & \left(n z^{n-1}\right)^{-k}\left[\mathfrak{f}^{(k)}(z)+\mathfrak{f}^{(k-1)}(z) O(1 /|z|)+\ldots\right] .
\end{aligned}
$$

Hence there exist functions $\mathfrak{a}_{j}(z)$, all rational at infinity and with $\mathfrak{a}_{k-1}(\infty)=0$, such that

$$
\mathfrak{f}^{(k)}(z)+\mathfrak{a}_{k-1}(z) \mathfrak{f}^{(k-1)}(z)+\ldots+\mathfrak{a}_{0}(z) \mathfrak{f}(z)=\mathfrak{F}(z)=\left(n z^{n-1}\right)^{k} F\left(z^{n}\right) .
$$

The new operator is $\mathfrak{L}=D^{k}+\mathfrak{a}_{k-1} D^{k-1}+\ldots$, and $y\left(z^{n}\right)$ solves $\mathfrak{L}[y]=0$ for every locally analytic or formal solution $y$ of $L[y]=0$, as does each $\mathfrak{f}_{j}(z)=f_{j}\left(z^{n}\right)$.

If $f$ is as in the hypotheses of Theorem 1.3 then running Frank's method as in Section 5 for $\mathfrak{f}$ and $\mathfrak{F}$ gives rise to auxiliary functions $\mathfrak{g}, \mathfrak{h}=-\left(\mathfrak{f}^{\prime} / \mathfrak{f}\right) \mathfrak{g}$ and $\mathfrak{w}_{j}$, which satisfy, using (5.1),

$$
\begin{aligned}
\mathfrak{g}(z)^{k} & =\frac{\mathfrak{f}(z)}{\mathfrak{F}(z)}=\frac{f\left(z^{n}\right)}{\left(n z^{n-1}\right)^{k} F\left(z^{n}\right)}=\frac{g\left(z^{n}\right)^{k}}{\left(n z^{n-1}\right)^{k}}, \quad \mathfrak{g}(z)=\frac{g\left(z^{n}\right)}{n z^{n-1}}, \\
\mathfrak{h}(z) & =-\frac{\mathfrak{f}^{\prime}(z)}{\mathfrak{f}(z)} \mathfrak{g}(z)=-n z^{n-1} \frac{f^{\prime}\left(z^{n}\right)}{f\left(z^{n}\right)} \frac{g\left(z^{n}\right)}{n z^{n-1}}=h\left(z^{n}\right), \\
\mathfrak{w}_{j}(z) & =\mathfrak{f}_{j}^{\prime}(z) \mathfrak{g}(z)+\mathfrak{f}_{j}(z) \mathfrak{h}(z)=f_{j}^{\prime}\left(z^{n}\right) g\left(z^{n}\right)+f_{j}\left(z^{n}\right) h\left(z^{n}\right)=w_{j}\left(z^{n}\right) .
\end{aligned}
$$

Thus the $\mathfrak{w}_{j}$ solve the equation $\mathfrak{M}[y]=y^{(k)}+\ldots=0$ which is obtained from $M[y]=0$ in the same way as $\mathfrak{L}[y]=0$ arose from $L[y]=0$. In $\mathfrak{M}[y]$ the coefficient of $y^{(k-1)}$ is $\mathfrak{a}_{k-1}$, since $a_{k-1}=A_{k-1}$. It is important to note that $\mathfrak{L}=\mathfrak{M}$ if and only if $L=M$.

Therefore $n$ may be chosen so that in the canonical formal solutions (4.1) for the equations $\mathfrak{L}[y]=0$ and $\mathfrak{M}[y]=0$ the integer $p$ is 1 . Moreover, $\mathfrak{L}[y]=0$ has linearly independent canonical formal solutions $\mathfrak{h}_{1}, \mathfrak{h}_{2}$ whose formal Wronskian $W\left(\mathfrak{h}_{1}, \mathfrak{h}_{2}\right)$ is free of logarithms. This is clear if there are solutions of $\mathfrak{L}[y]=0$ with distinct exponential parts. On the other hand if all exponential parts for $\mathfrak{L}[y]=0$ are the same then they are all 0 , since $\mathfrak{a}_{k-1}(\infty)=0$, and there exists a solution $\mathfrak{h}_{1}(z)=$ $z^{e_{1}} R_{1}(z) \not \equiv 0$, with $e_{1} \in \mathbf{C}$ and $R_{1}$ rational at infinity. The standard reduction of order method then gives an equation which is solved by $\left(y / \mathfrak{h}_{1}\right)^{\prime}=W\left(\mathfrak{h}_{1}, y\right) \mathfrak{h}_{1}^{-2}$, for every solution $y$ of $\mathfrak{L}[y]=0$, and which has a canonical formal solution free of logarithms.

When $L \neq M$, and hence $\mathfrak{L} \neq \mathfrak{M}$, Lemma 5.3 applied to $\mathfrak{L}$ and $\mathfrak{M}$ gives an equation $\mathfrak{N}[y]=0$, of order 2 or 3 , which is solved by $\mathfrak{g}$, as well as a counterpart $\mathfrak{V}$ for the operator $V$. Choosing $\mathfrak{h}_{1}, \mathfrak{h}_{2}$ as in the previous paragraph and any canonical formal solution $\mathfrak{G}$ of $\mathfrak{N}[y]=0$ then makes $\mathfrak{h}_{j}^{\prime} \mathfrak{G}+\mathfrak{h}_{j} \mathfrak{V}[\mathfrak{G}]$ a solution of $\mathfrak{M}[y]=0$. Solving for $\mathfrak{G}$ by Cramer's rule shows that in the canonical formal solutions (4.1) for $\mathfrak{N}[y]=0$ it may also be assumed that $p=1$.

\section{The main step}

Proposition 10.1. Assume that $f$ and $F$ are as in the hypotheses of Theorem 1.3, and that $f$ has finite order and an unbounded sequence of poles. Then there 
exist $E \in \mathbf{N}$ and a function $R$ which is rational at infinity such that all poles $z$ of $\mathfrak{f}(z)=f\left(z^{E}\right)$ with $|z|$ sufficiently large have multiplicity $R(z)$.

Once Proposition 10.1 is proved, Theorem 1.3 is established as follows. Under the hypotheses of Theorem 1.3, the first possibility is that $L=M$ and $f$ is determined by Proposition 6.1. If this is not the case then Lemma 5.3 shows that $f$ has finite order and an unbounded sequence of poles. In view of Proposition 10.1, Proposition 8.1 may be applied, and $f$ is thereby determined.

Assume for the remainder of the paper that the assumptions of Proposition 10.1 are satisfied.

Lemma 10.1. The following additional assumptions may all be made.

(A) In Section 5, the operators $L$ and $M$ are not the same.

(B) In the canonical formal solutions (4.1) for all of the equations $L[y]=0$, $M[y]=0$ and $N[y]=0$, the integer $p$ is 1 .

(C) The function $g$ solves no second order homogeneous linear differential equation with coefficients which are rational at infinity. Moreover, the operator $N$ has order 3 and may be written in the form

$$
N=D^{3}+B_{2} D^{2}+B_{1} D+B_{0},
$$

with the $B_{j}$ rational at infinity, while $\alpha \not \equiv 0$ in (5.16) and $D_{k-2}=C_{k-2}-$ $c_{k-2} \not \equiv 0$ in Section 5 .

Proof. Assumption (A) is legitimate because of Proposition 6.1, while (B) is justified by taking $\mathfrak{f}(z)=f\left(z^{m_{1}}\right)$ in place of $f$, for some $m_{1} \in \mathbf{N}$, as in Section 9 . Next, the first three assumptions of (C) are valid since otherwise (5.16) shows that $f$ and $g$ satisfy an equation (8.1) with $d_{0}$ and $d_{1}$ rational at infinity, in which case the conclusion of Proposition 10.1 follows from a comparison of residues. The last assumption of $(\mathrm{C})$ is justified by Proposition 6.1 .

Lemma 10.2. Assume that there exists a function $a^{*}$ which is rational at infinity, with the property that $-f^{\prime} / f+d g^{\prime} / g-a^{*}$ has no zeros in some $\Omega\left(r_{2}\right)$, where $d \in$ $\{0, \ldots, k-1\}$ is a constant. Then $d$ satisfies $d \neq(k-1) / 2$. Assume further that $a^{*}(\infty) \neq 0$. Then $g$ is given by

$$
P^{\prime} g=\beta_{1} e^{\omega_{1} P}+\beta_{2} e^{\omega_{2} P}+\beta_{3} e^{\omega_{3} P}, \quad \beta_{j}, \omega_{j} \in \mathbf{C}^{*}, \quad 1=\omega_{1} \neq \omega_{2} \neq \omega_{3} \neq 1,
$$

in which $P^{\prime}$ is rational at infinity, with $P^{\prime}(\infty) \neq 0$. If, in addition, $d=0$ or $d=k-1$ then $f$ satisfies the conclusion of Proposition 10.1.

Proof. As in (5.4), write $p^{\prime} / p=f^{\prime} / f+a_{k-1} / k$ and $q=-\left(p^{\prime} / p\right) g$. Then $g$ and $q$ solve the equations (5.7) to (5.12). Moreover, $a=a^{*}-a_{k-1} / k$ is rational at infinity and $-p^{\prime} / p+d g^{\prime} / g-a$ has no zeros in $\Omega\left(r_{2}\right)$. Since poles of $p^{\prime} / p$ have negative residues and are simple zeros of $g$, while $f^{\prime} / f$ and $g^{k}$ have finite order, it is possible to write

$$
q+d g^{\prime}-a g=g\left(-\frac{p^{\prime}}{p}+\frac{d g^{\prime}}{g}-a\right)=e^{P},
$$

with $P^{\prime}$ rational at infinity. Then (5.9) and (10.3) yield

$$
P^{\prime} e^{P}=x g^{\prime \prime}-a g^{\prime}-\left(\frac{D_{k-2}}{k}+a^{\prime}\right) g, \quad x=d-\frac{k-1}{2},
$$


and by Lemma $10.1(\mathrm{C})$ it may be assumed that $P^{\prime} \not \equiv 0$. Differentiation of this equation leads to

$$
\begin{aligned}
0= & x g^{\prime \prime \prime}+g^{\prime \prime}\left(-x\left(\frac{P^{\prime \prime}}{P^{\prime}}+P^{\prime}\right)-a\right)+g^{\prime}\left(a\left(\frac{P^{\prime \prime}}{P^{\prime}}+P^{\prime}\right)-\frac{D_{k-2}}{k}-2 a^{\prime}\right) \\
& +g\left(\left(\frac{P^{\prime \prime}}{P^{\prime}}+P^{\prime}\right)\left(\frac{D_{k-2}}{k}+a^{\prime}\right)-\frac{D_{k-2}^{\prime}}{k}-a^{\prime \prime}\right),
\end{aligned}
$$

and so $x \neq 0$ and $d \neq(k-1) / 2$, as asserted, again by Lemma 10.1(C).

Now assume that $a^{*}(\infty) \neq 0$, which implies that $a(\infty) \neq 0$. The following is an extension of a method from [7]. Since $C_{k-2}=D_{k-2}+c_{k-2}$, formula (5.10) becomes, in view of (10.3),

$$
\begin{aligned}
D_{k-2} e^{P}= & \frac{k\left(k^{2}-1\right)}{12} g^{\prime \prime \prime}+g^{\prime}\left((x+1) D_{k-2}+2 c_{k-2}\right) \\
& +g\left(\frac{k-1}{2} D_{k-2}^{\prime}+c_{k-2}^{\prime}-D_{k-3}-a D_{k-2}\right),
\end{aligned}
$$

and (5.12) may be written as

$$
\left(\frac{2 D_{k-3}}{k-2}-D_{k-2}^{\prime}\right) e^{P}=\frac{(k+2) D_{k-2}}{3} g^{\prime \prime}+d_{5} g^{\prime}+d_{6} g,
$$

with $d_{5}, d_{6}$ rational at infinity. Comparing the last equation with (10.4) delivers

$$
x\left(\frac{2 D_{k-3}}{k-2}-D_{k-2}^{\prime}\right)=\frac{(k+2) D_{k-2} P^{\prime}}{3},
$$

again using Lemma 10.1(C). Combining (10.4) with (10.6) and (10.7) leads to

$$
\begin{aligned}
0= & \frac{k\left(k^{2}-1\right)}{12} g^{\prime \prime \prime}-g^{\prime \prime}\left(\frac{x D_{k-2}}{P^{\prime}}\right)+g^{\prime}\left((x+1) D_{k-2}+2 c_{k-2}+\frac{a D_{k-2}}{P^{\prime}}\right) \\
& +g\left(\frac{D_{k-2}^{\prime}}{2}+c_{k-2}^{\prime}-\frac{\left(k^{2}-4\right) D_{k-2} P^{\prime}}{6 x}-a D_{k-2}+\frac{D_{k-2}}{P^{\prime}}\left(\frac{D_{k-2}}{k}+a^{\prime}\right)\right) .
\end{aligned}
$$

Lemma 10.1(C) implies that (10.8) must be (10.5) multiplied by $k\left(k^{2}-1\right) / 12 x$. Comparing the coefficients of $g^{\prime \prime}$ yields

$$
\frac{P^{\prime \prime}}{P^{\prime}}+P^{\prime}=-\frac{a}{x}+\frac{12 x D_{k-2}}{k\left(k^{2}-1\right) P^{\prime}}
$$

Next, matching the coefficients of $g^{\prime}$ and using (10.9) results in

$$
c_{k-2}=-\frac{\left(12 x^{2}+12 x+k^{2}-1\right) D_{k-2}}{24 x}-\frac{k\left(k^{2}-1\right) a^{\prime}}{12 x}-\frac{k\left(k^{2}-1\right) a^{2}}{24 x^{2}} .
$$

Examining the coefficients of $g$ in (10.5) and (10.8) in the light of (10.9) and (10.10) leads to

$$
a D_{k-2}\left(\frac{k^{2}-1-12 x^{2}}{12 x^{2}}\right)=D_{k-2}^{\prime}\left(\frac{12 x^{2}+1-k^{2}}{24 x}\right)+\frac{\left(k^{2}-4\right) D_{k-2} P^{\prime}}{6 x} .
$$

Because $k \geq 3$ and $D_{k-2} P^{\prime} \not \equiv 0$, this forces $k^{2}-1-12 x^{2} \neq 0$ and

$$
a=-\frac{x D_{k-2}^{\prime}}{2 D_{k-2}}+\frac{2 x\left(k^{2}-4\right) P^{\prime}}{k^{2}-1-12 x^{2}} \text {. }
$$


Therefore $P^{\prime}(\infty) \neq 0$, since $a(\infty) \neq 0$, and using (10.11) to eliminate $a$ from (10.9) delivers

$$
\frac{P^{\prime \prime}}{P^{\prime}}+\frac{\left(3 k^{2}-12 x^{2}-9\right) P^{\prime}}{k^{2}-1-12 x^{2}}-\frac{D_{k-2}^{\prime}}{2 D_{k-2}}=\frac{12 x D_{k-2}}{k\left(k^{2}-1\right) P^{\prime}} .
$$

Setting $Z=1 / D_{k-2}$ yields in turn a linear differential equation of form

$$
\left(\frac{2 P^{\prime \prime}}{P^{\prime}}+\eta_{1} P^{\prime}\right) Z+Z^{\prime}=\frac{24 x}{k\left(k^{2}-1\right) P^{\prime}}, \quad \eta_{1}=\frac{6\left(k^{2}-4 x^{2}-3\right)}{k^{2}-1-12 x^{2}} .
$$

If $k^{2}-4 x^{2}-3=0$, then $(k+2 x)(k-2 x)=3$; because $k, 2 x \in \mathbf{Z}$, this forces $2 k= \pm 4$, a contradiction. Assume henceforth that $k^{2}-4 x^{2}-3 \neq 0$ : then the integrating factor for $(10.12)$ is $\left(P^{\prime}\right)^{2} e^{\eta_{1} P}$, with $\eta_{1} \neq 0$, and the general solution to (10.12) is

$$
Z=\left(P^{\prime}\right)^{-2}\left(\eta_{2}+d_{7} e^{-\eta_{1} P}\right), \quad d_{7} \in \mathbf{C}, \quad \eta_{2}=\frac{24 x}{k\left(k^{2}-1\right) \eta_{1}} .
$$

Since $P^{\prime}$ and $Z$ are rational at infinity with $P^{\prime}(\infty) \neq 0$, this yields $Z=\eta_{2}\left(P^{\prime}\right)^{-2}$ and

$$
\begin{aligned}
\frac{-D_{k-2}}{k} & =\frac{-\left(P^{\prime}\right)^{2}}{k \eta_{2}}=\eta_{3}\left(P^{\prime}\right)^{2}, \\
\eta_{3} & =\frac{-\left(k^{2}-1\right) \eta_{1}}{24 x}=\frac{\left(4 x^{2}+3-k^{2}\right)\left(k^{2}-1\right)}{4 x\left(k^{2}-1-12 x^{2}\right)} \neq 0,
\end{aligned}
$$

as well as

$$
a=-x\left(\frac{P^{\prime \prime}}{P^{\prime}}+\eta_{4} P^{\prime}\right), \quad \eta_{4}=-\frac{2\left(k^{2}-4\right)}{k^{2}-1-12 x^{2}} \neq 0
$$

using (10.11). Combining (10.4), (10.13) and (10.14) shows that $g$ solves the equation

$$
P^{\prime} e^{P}=x\left(y^{\prime}+\left(\frac{P^{\prime \prime}}{P^{\prime}}+\eta_{4} P^{\prime}\right) y\right)^{\prime}+\eta_{3}\left(P^{\prime}\right)^{2} y .
$$

Now write $\zeta=P(z)$ and $Y_{0}(\zeta)=y(z) P^{\prime}(z)$ so that

$y^{\prime}+\left(\frac{P^{\prime \prime}}{P^{\prime}}+\eta_{4} P^{\prime}\right) y=\frac{d Y_{0}}{d \zeta}+\eta_{4} Y_{0}, \quad\left(y^{\prime}+\left(\frac{P^{\prime \prime}}{P^{\prime}}+\eta_{4} P^{\prime}\right) y\right)^{\prime}=P^{\prime}\left(\frac{d^{2} Y_{0}}{d \zeta^{2}}+\eta_{4} \frac{d Y_{0}}{d \zeta}\right)$.

Thus (10.15) becomes

$$
e^{\zeta}=x Y_{0}^{\prime \prime}(\zeta)+x \eta_{4} Y_{0}^{\prime}(\zeta)+\eta_{3} Y_{0}(\zeta)
$$

The auxiliary equation for the complementary function of (10.16) is then

$$
x \lambda^{2}+x \eta_{4} \lambda+\eta_{3}=0, \quad x \eta_{3} \eta_{4} \in \mathbf{C}^{*} .
$$

Suppose that 1 is a double root of (10.17). Then $g$ has a representation $g=$ $\left(P^{\prime}\right)^{-1} e^{P} Q_{2}(P)$, for some polynomial $Q_{2} \not \equiv 0$ of degree at most two. Since $P^{\prime}(\infty) \neq 0$, there cannot exist a sector on which $g$ has an unbounded sequence of zeros, contradicting the assumption that $f$ has an unbounded sequence of poles.

Now suppose that 1 is a simple root of (10.17), or that (10.17) has a repeated root. Then the fact that $\eta_{3} \neq 0$ gives

$$
P^{\prime} g=\left(\beta_{1}+\beta_{2} P\right) e^{\omega_{1} P}+\beta_{3} e^{\omega_{3} P}, \quad \beta_{j}, \omega_{j} \in \mathbf{C}, \quad 0 \neq \omega_{1} \neq \omega_{3} \neq 0 .
$$

Here $\beta_{2} \neq 0$ by Lemma 10.1(C), since otherwise $g$ satisfies a second order linear differential equation, and $\beta_{3} \neq 0$ by the assumption that $f$ has an unbounded sequence of poles. Denote by $\widehat{\psi}$ the result of analytically continuing a function element $\psi$ once 
around a given circle $|z|=r_{3}>r_{2}$. Then there exists $\zeta_{0} \in \mathbf{C}$ such that $\widehat{P}=P+\zeta_{0}$ and

$$
\begin{aligned}
\left(\beta_{1}+\beta_{2} \zeta_{0}+\beta_{2} P\right) e^{\omega_{1} P+\omega_{1} \zeta_{0}}+\beta_{3} e^{\omega_{3} P+\omega_{3} \zeta_{0}} & =P^{\prime} \widehat{g} \\
& =P^{\prime} \omega g=\omega\left(\left(\beta_{1}+\beta_{2} P\right) e^{\omega_{1} P}+\beta_{3} e^{\omega_{3} P}\right)
\end{aligned}
$$

where $\omega^{k}=1$. Because $\beta_{3} P^{\prime}(\infty) \neq 0$, examining the coefficients of $e^{\omega_{3} P}$ and $e^{\omega_{1} P}$ leads to

$$
e^{\omega_{3} \zeta_{0}}=\omega, \quad\left(\beta_{1}+\beta_{2} \zeta_{0}+\beta_{2} P\right) e^{\omega_{1} \zeta_{0}}=\omega\left(\beta_{1}+\beta_{2} P\right) .
$$

Differentiating the last relation then shows that $e^{\omega_{1} \zeta_{0}}=\omega$, since $\beta_{2} \neq 0$, and

$$
\omega \beta_{1}=\left(\beta_{1}+\beta_{2} \zeta_{0}\right) e^{\omega_{1} \zeta_{0}}=\omega\left(\beta_{1}+\beta_{2} \zeta_{0}\right),
$$

so that $\zeta_{0}=0$ and $P$ is rational at infinity, which forces $g$ to solve a second order equation, contradicting Lemma 10.1(C). Thus (10.2) holds, with the $\omega_{j} \in \mathbf{C}^{*}$ pairwise distinct, since $\eta_{3} \neq 0$, and $\omega_{1}=1$, and none of the $\beta_{j}$ can vanish, again by Lemma $10.1(\mathrm{C})$. The proof of (10.2) is now complete.

Next, suppose that $d=0$ or $d=k-1$, so that $x= \pm(k-1) / 2$. Now (10.13) and (10.14) imply that (10.16) takes the form

$$
e^{\zeta}=x Y_{0}^{\prime \prime}(\zeta)+x\left(\frac{k+2}{k-1}\right) Y_{0}^{\prime}(\zeta)+x\left(\frac{k+1}{(k-1)^{2}}\right) Y_{0}(\zeta)
$$

The auxiliary equation for the complementary function has roots $\lambda_{j}=1-j k /(k-1)$, for $j=1,2$, and (10.2) becomes, in view of Lemma 10.1(C),

$$
g=\frac{e^{P}}{P^{\prime}}\left(e_{1}+e_{2} e^{\eta P}+e_{3} e^{2 \eta P}\right), \quad \eta=-\frac{k}{k-1}, \quad e_{j} \in \mathbf{C}^{*} .
$$

Thus (5.4), (10.3), (10.18) and partial fractions deliver

$$
\begin{gathered}
g=\frac{e_{4} e^{P}}{P^{\prime}}\left(e^{\eta P}-e_{5}\right)\left(e^{\eta P}-e_{6}\right), \\
\frac{f^{\prime}}{f}+\frac{a_{k-1}}{k}+a=\frac{p^{\prime}}{p}+a=e_{7} P^{\prime}\left(\frac{1}{e^{\eta P}-e_{5}}-\frac{1}{e^{\eta P}-e_{6}}\right)+\frac{d g^{\prime}}{g}, \quad e_{j} \in \mathbf{C} .
\end{gathered}
$$

Again the $e_{j}$ are all non-zero, and $e_{5} \neq e_{6}$ since $g$ cannot have multiple zeros. If $r_{4}$ is large and some continuation of $e^{\eta P}$ takes the value $e_{5}$ at some $z_{0} \in \Omega\left(r_{4}\right)$ then $z_{0}$ is a zero of $g$, and so is a pole of $f$ of multiplicity $m_{1}$ satisfying $-m_{1}=d+e_{7} / \eta e_{5}$, so that $(5.2),(10.18)$ and (10.19) imply that at the point $z_{0}$ the following equations are satisfied:

$$
\begin{aligned}
e_{5}^{k-1} & =e^{(k-1) \eta P}=e^{-k P} \\
\frac{(-1)^{k}}{m_{1}\left(m_{1}+1\right) \ldots\left(m_{1}+k-1\right)} & =\left(g^{\prime}\right)^{k}=\left(e_{4} e^{P} \eta e_{5}\left(e_{5}-e_{6}\right)\right)^{k} \\
& =e_{4}^{k} e_{5}^{1-k} \eta^{k} e_{5}^{k}\left(e_{5}-e_{6}\right)^{k}=e_{4}^{k} e_{5} \eta^{k}\left(e_{5}-e_{6}\right)^{k} .
\end{aligned}
$$

Similarly, all zeros of continuations of $e^{\eta P}-e_{6}$ to $\Omega\left(r_{4}\right)$ are poles of $f$ of multiplicity $m_{2}$, where $-m_{2}=d-e_{7} / \eta e_{6}$, and

$$
\frac{m_{2}\left(m_{2}+1\right) \ldots\left(m_{2}+k-1\right)}{m_{1}\left(m_{1}+1\right) \ldots\left(m_{1}+k-1\right)}=(-1)^{k} \frac{e_{5}}{e_{6}}=(-1)^{k-1} \frac{m_{2}+d}{m_{1}+d} .
$$

But $d=0$ or $d=k-1$, so that $m_{1}=m_{2}$ (and $k$ is odd). 


\section{The exponential parts for the equation $N[y]=0$}

Let $p$ and $q$ be polynomials in $z$, and let $\theta \in \mathbf{R}$. Write $p \prec q$ (respectively, $p \preceq q$, $p \simeq q)$ to indicate that $\operatorname{Re} p\left(r e^{i \theta}\right)<\operatorname{Re} q\left(r e^{i \theta}\right)\left(\right.$ respectively $\operatorname{Re} p\left(r e^{i \theta}\right) \leq \operatorname{Re} q\left(r e^{i \theta}\right)$, $\left.\operatorname{Re} p\left(r e^{i \theta}\right)=\operatorname{Re} q\left(r e^{i \theta}\right)\right)$ as $r \rightarrow+\infty$. Since each $P_{\theta}(r)=\operatorname{Re} p\left(r e^{i \theta}\right)$ is a polynomial in $r$, every $\theta \in \mathbf{R}$ has $p \prec 0$ or $p \simeq 0$ or $0 \prec p$, and if $p$ is not constant then all but finitely many $\theta \in[0,2 \pi]$ have either $p \prec 0$ or $0 \prec p$.

Suppose that $N[y]=0$ has linearly independent canonical formal solutions with exponential parts $\kappa_{1}, \kappa_{2}, \kappa_{3}$. The $\kappa_{j}$ are polynomials in $z$ by Lemma 10.1, and it will be assumed as before that $\kappa_{j}(0)=0$ for all $j$, from which it follows that if $\kappa_{j}-\kappa_{j^{\prime}}$ is constant then $\kappa_{j}-\kappa_{j^{\prime}} \equiv 0$.

Lemma 11.1. The $\kappa_{j}$ are not all the same polynomial, and there does not exist $\theta \in \mathbf{R}$ with $\kappa_{j} \prec 0$ on $\arg z=\theta$ for $j=1,2,3$.

Proof. The first assertion is proved in Lemma 7.2, and the second holds because otherwise $g^{k}=f / F$ tends to zero transcendentally fast on a sector, contradicting Lemma 7.2.

Lemma 11.1 does not exclude two of the $\kappa_{j}$ being the same polynomial, possibly identically zero, and this case will be dealt with in Sections 15 and 18. When there is no repetition among the $\kappa_{j}$, the next lemma shows that there are two subcases to handle.

Lemma 11.2. Suppose that the $\kappa_{j}$ are pairwise distinct. Then it is possible to label the $\kappa_{j}$ and choose a ray $\arg z=\theta \in \mathbf{R}$ such that

$$
\text { (A) } \quad \kappa_{1} \prec \kappa_{2} \prec 0 \prec \kappa_{3} \quad \text { or } \quad(\mathrm{B}) \quad \kappa_{1} \prec \kappa_{2} \prec 0, \quad \kappa_{3} \equiv 0
$$

or

$$
\text { (C) } \kappa_{1} \prec 0 \prec \kappa_{3}, \quad \kappa_{2} \equiv 0 .
$$

Proof. If one of the $\kappa_{j}$ is identically zero label the other two as $\kappa_{a}$ and $\kappa_{b}$, and choose $\theta \in[0,2 \pi]$ such that $\kappa_{a} \prec 0$ on $\arg z=\theta$. A small change to $\theta$ delivers either $\kappa_{b} \prec \kappa_{a}$, which leads to (B), or $\kappa_{a} \prec \kappa_{b} \prec 0$ or $\kappa_{a} \prec 0 \prec \kappa_{b}$, leading to (B) or (C).

Assume now that none of the $\kappa_{j}$ is the zero polynomial. Let $m^{*}$ be the largest of the degrees of the $\kappa_{j}$ and, with no loss of generality, write

$$
\kappa_{j}(z)=\alpha_{j} z^{m^{*}}+\ldots, \quad \alpha_{1} \neq 0 .
$$

If $\alpha_{2}=0$ then it is easy to choose a ray $\arg z=\theta$ on which $\kappa_{1} \prec \kappa_{2} \prec 0$ and, by varying $\theta$ slightly if necessary, either $\kappa_{3} \prec 0$ or $0 \prec \kappa_{3}$. Lemma 11.1 then implies that (A) must hold.

Next, suppose that $\alpha_{j} \neq 0$ for all $j$. If $\alpha_{2} / \alpha_{1}$ is not a negative real number choose a ray on which $\alpha_{1} z^{m^{*}}$ and $\alpha_{2} z^{m^{*}}$ both have negative real part and $\kappa_{3} \neq 0$. Shifting $\theta$ slightly gives either $\kappa_{1} \prec \kappa_{2} \prec 0$ or $\kappa_{2} \prec \kappa_{1} \prec 0$, and Lemma 11.1 forces (A) to hold, subject to re-labelling if necessary.

Thus the proof is complete, after re-labelling if necessary, unless both $\alpha_{2} / \alpha_{1}$ and $\alpha_{3} / \alpha_{1}$ are negative real numbers, in which case the argument of the previous paragraph applies with $\kappa_{2}$ and $\kappa_{3}$ in place of $\kappa_{1}$ and $\kappa_{2}$.

\section{A decomposition of the operators $N$ and $V$}

By Lemma 10.1(C), the equation $N[y]=0$ in Lemma 5.3, which is satisfied by $g$, has order 3, and so the asymptotics for its solutions may be complicated. However, 
the following lemma gives a condition under which two linearly independent solutions of $N[y]=0$ must together solve a second order equation, for which the asymptotics are then considerably simpler.

Lemma 12.1. With $N$ and $V$ as in Lemma 5.3, suppose that $g_{1}$ and $g_{2}$ are linearly independent (both formal or both locally analytic) solutions of $N[y]=0$ such that

$$
g_{1} V\left[g_{2}\right]-g_{2} V\left[g_{1}\right]=d\left(g_{2} g_{1}^{\prime}-g_{1} g_{2}^{\prime}\right),
$$

where $d$ is rational at infinity. Then $g_{1}$ and $g_{2}$ solve an equation (3.4) and

$$
\begin{gathered}
W^{\prime}+E_{1} W=0, \quad E_{1}=\frac{\beta+d}{\alpha}, \quad W=W\left(g_{1}, g_{2}\right), \\
N=(D+\delta) \circ\left(D^{2}+E_{1} D+E_{0}\right),
\end{gathered}
$$

where $E_{1}, E_{0}$ and $\delta$ are rational at infinity. If, in addition, $d$ is constant then

$$
V=\alpha\left(D^{2}+E_{1} D+E_{0}\right)-d D-x E_{1}-\frac{X^{\prime}}{X}, \quad x=d-\frac{k-1}{2}, \quad \frac{X^{\prime}}{X}=-\frac{a_{k-1}}{k} .
$$

Proof. Differentiating $W=W\left(g_{1}, g_{2}\right)=g_{1} g_{2}^{\prime}-g_{1}^{\prime} g_{2}$ gives $W^{\prime}=g_{1} g_{2}^{\prime \prime}-g_{1}^{\prime \prime} g_{2}$. Thus equation (12.1) can be rewritten, using (5.16), in the form (12.2), with $E_{1}$ rational at infinity. Applying Lemma 3.4 shows that $g_{1}$ and $g_{2}$ solve an equation (3.4), with $E_{0}$ also rational at infinity. Because $g_{1}$ and $g_{2}$ are linearly independent solutions of $N[y]=0$ and (3.4), the operator $N$ factorises using (10.1) and the division algorithm for linear differential operators [15, p. 126] as

$$
\begin{aligned}
N & =D^{3}+B_{2} D^{2}+B_{1} D+B_{0}=(D+\delta) \circ\left(D^{2}+E_{1} D+E_{0}\right) \\
& =D^{3}+\left(E_{1}+\delta\right) D^{2}+\left(E_{0}+E_{1}^{\prime}+\delta E_{1}\right) D+E_{0}^{\prime}+\delta E_{0},
\end{aligned}
$$

where $\delta$ is again rational at infinity.

Now suppose that $d$ is constant. (5.15) and (5.16) yield

$h^{\prime}=\alpha g^{\prime \prime \prime}+\left(\beta+\alpha^{\prime}\right) g^{\prime \prime}+\left(\beta^{\prime}+\gamma\right) g^{\prime}+\gamma^{\prime} g=-\left(\frac{k-1}{2}\right) g^{\prime \prime}+\frac{a_{k-1}}{k} g^{\prime}+\frac{a_{k-1}^{\prime}-D_{k-2}}{k} g$,

where $D_{k-2}$ is rational at infinity, so that

$$
0=\alpha g^{\prime \prime \prime}+\left(\beta+\alpha^{\prime}+\frac{k-1}{2}\right) g^{\prime \prime}+\left(\beta^{\prime}+\gamma-\frac{a_{k-1}}{k}\right) g^{\prime}+\left(\gamma^{\prime}+\frac{D_{k-2}-a_{k-1}^{\prime}}{k}\right) g .
$$

Comparing coefficients with (12.5) leads, using Lemma 10.1(C), to

$$
E_{1}+\delta=\frac{\beta+\alpha^{\prime}+(k-1) / 2}{\alpha}, \quad E_{0}+E_{1}^{\prime}+\delta E_{1}=\frac{\beta^{\prime}+\gamma-a_{k-1} / k}{\alpha} .
$$

Now (12.2) and (12.6) deliver, with $x=d-(k-1) / 2$,

$$
\begin{aligned}
\beta & =\alpha E_{1}-d, \quad \delta=\frac{\beta+\alpha^{\prime}+(k-1) / 2}{\alpha}-E_{1}=\frac{\alpha^{\prime}}{\alpha}-\frac{x}{\alpha}, \\
\gamma & =\alpha\left(E_{0}+E_{1}^{\prime}+\delta E_{1}\right)-\beta^{\prime}+\frac{a_{k-1}}{k}=\alpha\left(E_{0}+E_{1}^{\prime}+\delta E_{1}\right)-\alpha^{\prime} E_{1}-\alpha E_{1}^{\prime}+\frac{a_{k-1}}{k} \\
& =\alpha E_{0}+E_{1}\left(\alpha^{\prime}-x\right)-\alpha^{\prime} E_{1}+\frac{a_{k-1}}{k}=\alpha E_{0}-x E_{1}+\frac{a_{k-1}}{k},
\end{aligned}
$$

and the representations given here for $\beta$ and $\gamma$ yield (12.4). 
Lemma 12.2. Suppose that $g_{1}$ and $g_{2}$ are linearly independent (both formal or both locally analytic) solutions of $N[y]=0$, such that (12.1) holds, with $d \in$ $\{0, \ldots, k-1\}$ a constant. Then $g_{1}$ and $g_{2}$ solve an equation (3.4), and formulas (12.2) to (12.4) hold, with $E_{1}, E_{0}$ and $\delta$ rational at infinity, and $d$ satisfies $d \neq(k-1) / 2$. Suppose further that $E_{1}(\infty) \neq 0$ in (3.4). Then $g$ is given by (10.2). If, in addition, $d=0$ or $d=k-1$, then $f$ satisfies the conclusion of Proposition 10.1.

Proof. Lemma 12.1 gives the equation (3.4) solved by $g_{1}$ and $g_{2}$, as well as formulas (12.2) to (12.4). Since $N[g]=0$, but $g^{\prime \prime}+E_{1} g^{\prime}+E_{0} g \not \equiv 0$, (5.16), (12.3) and (12.4) deliver

$$
g^{\prime \prime}+E_{1} g^{\prime}+E_{0} g=e^{\tau}, \quad \tau^{\prime}=-\delta,
$$

and

$$
-\frac{f^{\prime}}{f}=\frac{h}{g}=\frac{V[g]}{g}=\frac{\alpha e^{\tau}}{g}-\frac{d g^{\prime}}{g}-x E_{1}+\frac{a_{k-1}}{k}=\frac{\alpha e^{\tau}}{g}-\frac{d g^{\prime}}{g}+a^{*} .
$$

Here the function $a^{*}=-x E_{1}+a_{k-1} / k$ is rational at infinity and $-f^{\prime} / f+d g^{\prime} / g-a^{*}$ continues without zeros in some $\Omega\left(r_{2}\right)$. Hence Lemma 10.2 and (12.4) imply that $d \neq(k-1) / 2$ and $x \neq 0$. Finally, if $E_{1}(\infty) \neq 0$ in (3.4) then $a^{*}(\infty) \neq 0$ and the remaining assertions of Lemma 12.2 follow from Lemma 10.2.

\section{Analytic solutions decaying in the same sector}

This section determines conditions under which Lemma 12.2 may be applied with analytic solutions of $N[y]=0$.

Lemma 13.1. Assume that there exist linearly independent analytic solutions $g_{1}, g_{2}$ of $N[y]=0$, such that both tend to 0 transcendentally fast as $z \rightarrow \infty$ in the same sector $S$. Then $g_{1}$ and $g_{2}$ satisfy the hypotheses of Lemma 12.2, for some constant $d \in\{0, \ldots, k-1\}$, and solve an equation (3.4), with $E_{1}$ and $E_{0}$ rational at infinity and $E_{1}(\infty) \neq 0$. Moreover, formulas (12.2) to (12.4) hold, and $d$ and $g$ satisfy $d \neq(k-1) / 2$ and (10.2). Finally, if $d=0$ or $d=k-1$ then the conclusion of Proposition 10.1 holds.

In the context of Section 11, Lemma 13.1 applies if there is a repeated non-trivial exponential part among the $\kappa_{j}$, or if (11.1) holds for some ray $\arg z=\theta$.

Proof. Choose $z_{0} \in S$ such that $z_{0}$ is not a singular point for any of the operators $L, M, N$, and such that

$$
g_{1}\left(z_{0}\right) g_{2}\left(z_{0}\right) \neq 0, \quad W\left(g_{1}, g_{2}\right)\left(z_{0}\right) \neq 0 .
$$

Let $w$ lie close to $z_{0}$. Then $g_{w}(z)=g_{2}(w) g_{1}(z)-g_{1}(w) g_{2}(z)$ tends to 0 transcendentally fast as $z \rightarrow \infty$ in $S$ and, by Lemma $7.2, g_{w}$ annihilates a solution $f_{w} \not \equiv 0$ of $L[y]=0$, with

$$
\frac{f_{w}^{\prime}(z)}{f_{w}(z)}=-\frac{V\left[g_{w}\right](z)}{g_{w}(z)}=\frac{g_{1}(w) V\left[g_{2}\right](z)-g_{2}(w) V\left[g_{1}\right](z)}{g_{2}(w) g_{1}(z)-g_{1}(w) g_{2}(z)} .
$$

Let

$$
G_{0}(z)=\frac{g_{1}(z) V\left[g_{2}\right](z)-g_{2}(z) V\left[g_{1}\right](z)}{g_{2}(z) g_{1}^{\prime}(z)-g_{1}(z) g_{2}^{\prime}(z)}
$$

The second condition of (13.1) implies that $w$ is a simple zero of $g_{w}$, and by (13.2) the residue of $f_{w}^{\prime} / f_{w}$ at $w$ is $G_{0}(w)$, which must belong to the set $\{0, \ldots, k-1\}$. Since this holds for all $w$ near $z_{0}$, the function $G_{0}$ is a constant $d \in\{0, \ldots, k-1\}$, and so $g_{1}$ and $g_{2}$ satisfy the hypotheses of Lemma 12.2, and hence solve an equation (3.4). Cauchy's estimate for derivatives shows that $W\left(g_{1}, g_{2}\right)$ tends to 0 transcendentally 
fast in a subsector of $S$, which gives $E_{1}(\infty) \neq 0$ by Abel's identity. The remaining assertions hold by Lemma 12.2.

\section{Decaying solutions with different exponential parts}

This section will deal with one case of the situation in Section 13, in which two linearly independent solutions of $N[y]=0$ decay in the same sector and have different exponential parts, corresponding to (11.1) in Lemma 11.2. The case of a repeated non-trivial exponential part will be addressed in Section 15. The methods of this section are heavily influenced by [2], but a decisive role will be played by Lemma 13.1 and the second order equation (3.4).

Proposition 14.1. Assume that there exists a ray $\arg z=\theta$ on which the exponential parts $\kappa_{j}$ for the equation $N[y]=0$ satisfy (11.1). Then $f$ satisfies the conclusions of Proposition 10.1.

To prove Proposition 14.1, note first that if $\theta$ is varied slightly, then (11.1) continues to hold. Take canonical formal solutions $g_{1}, g_{2}$ of $N[y]=0$ with exponential parts $\kappa_{1}, \kappa_{2}$. By (11.1) the exponential parts for $N[y]=0$ are pairwise distinct, and there exist linearly independent analytic solutions $G_{1}, G_{2}$ of $N[y]=0$ which are asymptotic to $g_{1}$ and $g_{2}$ respectively on a sector centred on the ray $\arg z=\theta$, and so tend to 0 transcendentally fast there, by (11.1). Thus the hypotheses of Lemma 13.1 are satisfied, and therefore so are those of Lemma 12.2, for some $d$ in $\{0, \ldots, k-1\}$, which gives rise to an equation (3.4) satisfied by the $G_{j}$. Computing series representations for $0=G_{j}^{\prime \prime}+E_{1} G_{j}^{\prime}+E_{0} G_{j}$ shows that the $g_{j}$ also solve (3.4). Thus the exponential parts $\kappa_{1}$ and $\kappa_{2}$ correspond to the equation (3.4), while $\kappa_{3}$ is from the third canonical formal solution of $N[y]=0$. Furthermore, $V$ satisfies (12.4).

Next, let the operators $L, M$ have canonical formal solutions with exponential parts $q_{j}, s_{j}$ respectively, labelled so that

$$
q_{1} \preceq q_{2} \preceq \ldots \preceq q_{k}, \quad s_{1} \preceq s_{2} \preceq \ldots \preceq s_{k},
$$

on $\arg z=\theta$ (the last phrase will be omitted henceforth). The $q_{j}$ and $s_{j}$ are polynomials in $z$ with zero constant term. It may be assumed that $\theta$ is chosen so that if $\widetilde{p}_{1}, \widetilde{p}_{2} \in\left\{q_{1}, \ldots, q_{k}, s_{1}, \ldots, s_{k}\right\}$ and $\widetilde{p}_{1}-\widetilde{p}_{2} \not \equiv 0$ then $\widetilde{p}_{1} \prec \widetilde{p}_{2}$ or $\widetilde{p}_{2} \prec \widetilde{p}_{1}$.

Lemma 14.1. There exists $\lambda \in\{1, \ldots, k\}$ such that the canonical formal solution $g_{1}$ of (3.4) with exponential part $\kappa_{1}$ annihilates a canonical formal solution $f_{\lambda}$ of $L[y]=0$ with exponential part $q_{\lambda}$, and the exponential parts for $M[y]=0$ are

$$
q_{j}+\kappa_{1} \quad(j \neq \lambda), \quad q_{\lambda}-(k-1) \kappa_{1} .
$$

Moreover, this $f_{\lambda}$ may be assumed to be $g_{1}^{d} W^{-x} X$, where $W, x$ and $X$ are as in Lemma 12.1. Furthermore, there exists $\mu \in\{1, \ldots, k\}$ such that the canonical formal solution $g_{2}$ of (3.4) with exponential part $\kappa_{2}$ annihilates a canonical formal solution $f_{\mu}=g_{2}^{d} W^{-x} X$ of $L[y]=0$, with exponential part $q_{\mu}$, while the exponential parts for $M[y]=0$ are

$$
q_{j}+\kappa_{2} \quad(j \neq \mu), \quad q_{\mu}-(k-1) \kappa_{2} .
$$

Proof. Since the exponential parts for $N[y]=0$ are pairwise distinct, $g_{1}$ and $g_{2}$ both have non-zero exponential parts and are free of logarithms. Thus Lemma 7.1 gives a canonical formal solution $f_{\lambda}$ of $L[y]=0$, with exponential part $q_{\lambda}$, such that $g_{1}$ annihilates $f_{\lambda}$ and the exponential parts for $M[y]=0$ are given by (14.2). Moreover, since $g_{1}$ is a solution of (3.4), solving $0=f_{\lambda}^{\prime} g_{1}+f_{\lambda} V\left[g_{1}\right]$ in the light of (12.4) shows 
that $f_{\lambda}$ is a constant multiple of $g_{1}^{d} W^{-x} X$. The same argument works for $g_{2}$ and $f_{\mu}$.

Lemma 14.2. The integer $\lambda$ is 1 .

Proof. Suppose not: then an exponential part $q_{1}+\kappa_{1}$ occurs in the list (14.2). But this term, in view of (11.1) and (14.1), cannot be realised as $q_{j}+\kappa_{2}$ or $q_{\mu}-(k-$ 1) $\kappa_{2}$.

Lemma 14.3. The $q_{j}$ satisfy $q_{j}+\kappa_{1} \preceq q_{1}-(k-1) \kappa_{1}$ for $2 \leq j \leq k$.

Proof. Suppose that this is not the case. Then the term $q_{k}+\kappa_{1}$, which does occur in the list (14.2), must be maximal according to the ordering $\preceq$. But (11.1) implies that

$$
q_{k}+\kappa_{1} \prec q_{k}+\kappa_{2} \prec q_{k}-(k-1) \kappa_{2} .
$$

This is a contradiction since the second or third term in (14.4) occurs in the list (14.3).

Thus by (14.2) the $s_{j}$ in (14.1) can now be written as

$$
s_{1}=q_{2}+\kappa_{1}, \quad \ldots, \quad s_{k-1}=q_{k}+\kappa_{1}, \quad s_{k}=q_{1}-(k-1) \kappa_{1} .
$$

Note that each of these relations initially holds with $\simeq$ in place of $=$, but may be assumed to be an identity, by the remark following (14.1). The same property will subsequently be used on a number of occasions without explicit reference.

Lemma 14.4. The exponential part $s_{\mu}$ satisfies $s_{\mu}=q_{\mu}-(k-1) \kappa_{2}$.

Proof. Suppose first that $q_{\mu}-(k-1) \kappa_{2} \prec s_{\mu}$. Then (11.1) and (14.1) give $\mu>1$ and

$$
q_{1}+\kappa_{2} \preceq \ldots \preceq q_{\mu-1}+\kappa_{2} \prec q_{\mu}-(k-1) \kappa_{2} \prec s_{\mu},
$$

in which all of the first $\mu$ terms occur in the list (14.3). Hence the second list in (14.1) includes $\mu$ terms $\widetilde{s}$ all satisfying $\widetilde{s} \prec s_{\mu}$, which is a contradiction.

Now suppose that $s_{\mu} \prec q_{\mu}-(k-1) \kappa_{2}$. Then $\mu<k$ and in the list (14.3) there are at least $\mu$ terms $\widetilde{s}$ all satisfying $\widetilde{s} \preceq s_{\mu} \prec q_{\mu}-(k-1) \kappa_{2}$. Of these, $\mu-1$ are $q_{1}+\kappa_{2}, \ldots, q_{\mu-1}+\kappa_{2}$ (this list being void if $\mu=1$ ), and it must be the case that $q_{\mu+1}+\kappa_{2} \preceq s_{\mu} \prec q_{\mu}-(k-1) \kappa_{2}$. But then (11.1) and (14.5) yield a contradiction via

$$
s_{\mu}=q_{\mu+1}+\kappa_{1} \prec q_{\mu+1}+\kappa_{2} \preceq s_{\mu} .
$$

Lemma 14.4 implies that among the $q_{j}+\kappa_{2}(j \neq \mu)$ there are at least $\mu-1$ terms $\widetilde{s}$ with $\widetilde{s} \preceq q_{\mu}-(k-1) \kappa_{2}$, and if $\mu>1$ these must include $q_{1}+\kappa_{2}, \ldots, q_{\mu-1}+\kappa_{2}$; similarly, there are at least $k-\mu$ terms with $q_{\mu}-(k-1) \kappa_{2} \preceq \widetilde{s}$, and if $\mu<k$ these must include $q_{\mu+1}+\kappa_{2}, \ldots, q_{k}+\kappa_{2}$. It follows that

$$
s_{j}=q_{j}+\kappa_{2} \quad(j \neq \mu), \quad s_{\mu}=q_{\mu}-(k-1) \kappa_{2} .
$$

Lemma 14.5. The integers $d$ and $\mu$ are related by $d=\mu-1$.

Proof. By Lemmas 14.1 and 14.2 the canonical formal solutions $f_{\lambda}$ and $f_{\mu}$ of $L[y]=0$ annihilated by $g_{1}$ and $g_{2}$ have exponential parts $q_{1}$ and $q_{\mu}$ respectively. The quotient $f_{\mu} / f_{\lambda}=\left(g_{2} / g_{1}\right)^{d}$ has exponential part $d\left(\kappa_{2}-\kappa_{1}\right)$, which implies that $d\left(\kappa_{2}-\kappa_{1}\right)=q_{\mu}-q_{1}$. If $\mu=1$ this gives $d=0$ since $\kappa_{1} \neq \kappa_{2}$. For $\mu>1$, (14.5) and (14.6) yield

$$
s_{1}=q_{2}+\kappa_{1}, \quad \ldots, \quad s_{\mu-1}=q_{\mu}+\kappa_{1}, \quad s_{1}=q_{1}+\kappa_{2}, \quad \ldots, \quad s_{\mu-1}=q_{\mu-1}+\kappa_{2},
$$


and so

$$
\kappa_{2}-\kappa_{1}=q_{2}-q_{1}=\ldots=q_{\mu}-q_{\mu-1}, \quad d\left(\kappa_{2}-\kappa_{1}\right)=q_{\mu}-q_{1}=(\mu-1)\left(\kappa_{2}-\kappa_{1}\right) .
$$

By (11.1) there exists a canonical formal solution $g_{3}$ of $N[y]=0$ which is free of logarithms and has exponential part $\kappa_{3}$.

Lemma 14.6. The exponential part $\kappa_{3}$ is not the zero polynomial, and case $(A)$ applies in (11.1).

Proof. Suppose that $\kappa_{3} \equiv 0$. Then Lemma 7.1 and (14.6) give at least one $j$ with $q_{j}=s_{j}=q_{j}+\kappa_{2} \prec q_{j}$, a contradiction.

By Lemmas 7.1 and 14.6, there exists $\nu$ such that $g_{3}$ annihilates a canonical formal solution of $L[y]=0$ with exponential part $q_{\nu}$, and the exponential parts for $M[y]=0$ are

$$
q_{j}+\kappa_{3} \quad(j \neq \nu), \quad q_{\nu}-(k-1) \kappa_{3} .
$$

Lemma 14.7. Assume that $2 \leq \mu \leq k-1$. Then $\nu=k$ and $s_{1}=q_{k}-(k-1) \kappa_{3}$.

Proof. Suppose first that $\nu<k$. Then the list (14.7) includes $q_{k}+\kappa_{3}$, which must be maximal with respect to the ordering $\preceq$, since $0 \prec \kappa_{3}$. But $\mu \neq k$ by assumption, which gives

$$
q_{k}+\kappa_{3}=s_{k}=q_{k}+\kappa_{2}
$$

using (14.6), and this contradicts (11.1). Thus $\nu=k$ in (14.7).

Now suppose that $s_{1} \neq q_{k}-(k-1) \kappa_{3}$. Then $s_{1} \prec q_{k}-(k-1) \kappa_{3}$ and so $s_{1}=q_{1}+\kappa_{3}$, whereas (14.6) gives $s_{1}=q_{1}+\kappa_{2}$ since $\mu \neq 1$, again contradicting (11.1).

Lemma 14.8. If $k \geq 4$ then $\mu=1$ or $\mu=k$.

Proof. Suppose instead that $2 \leq \mu \leq k-1$. Then, by Lemma 14.7, the list (14.7) consists of

$$
s_{1}=q_{k}-(k-1) \kappa_{3}, \quad s_{2}=q_{1}+\kappa_{3}, \quad \ldots, \quad s_{k}=q_{k-1}+\kappa_{3} .
$$

Using (14.5), (14.6) and (14.8) gives

$$
s_{\mu+1}=q_{\mu}+\kappa_{3}=q_{\mu+1}+\kappa_{2}, \quad q_{\mu}-q_{\mu+1}=\kappa_{2}-\kappa_{3},
$$

and

$$
s_{\mu}=q_{\mu+1}+\kappa_{1}=q_{\mu}-(k-1) \kappa_{2}, \quad q_{\mu}-q_{\mu+1}=\kappa_{1}+(k-1) \kappa_{2} .
$$

Define $\tau$ as follows: if $2 \leq \mu \leq k-2$ take $\tau=k-1$, and if $\mu=k-1$ choose $\tau=1$. In either case $\mu \neq \tau, \tau+1$, since $k \geq 4$ by assumption. Thus (14.5), (14.6) and (14.8) deliver

$$
s_{\tau+1}=q_{\tau+1}+\kappa_{2}=q_{\tau}+\kappa_{3}, \quad q_{\tau+1}-q_{\tau}=\kappa_{3}-\kappa_{2},
$$

in addition to

$$
s_{\tau}=q_{\tau}+\kappa_{2}=q_{\tau+1}+\kappa_{1}, \quad q_{\tau+1}-q_{\tau}=\kappa_{2}-\kappa_{1} .
$$

Combining (14.9), (14.10), (14.11) and (14.12) yields

$$
\kappa_{2}-\kappa_{1}=\kappa_{3}-\kappa_{2}=-\kappa_{1}-(k-1) \kappa_{2},
$$

contradicting the fact that $\kappa_{2} \prec 0$.

Thus $d$ must be 0 or $k-1$ : this follows from Lemmas 14.5 and 14.8 when $k \geq 4$, while if $k=3$ then Lemma 13.1 forces $d \neq(k-1) / 2=1$. Hence the 
conclusion of Proposition 10.1 holds by Lemma 13.1 and the proof of Proposition 14.1 is complete.

\section{The case of a repeated non-trivial exponential part}

Suppose that $\kappa$ is a repeated non-trivial exponential part for the equation $N[y]=$ 0 . Then it is possible to choose a ray $\arg z=\theta \in \mathbf{R}$ on which $\kappa \prec 0$, and linearly independent analytic solutions $g_{1}, g_{2}$ of $N[y]=0$, each with exponential part $\kappa$ near $\arg z=\theta$. It then follows from Lemma 13.1 that $g$ is given by (10.2), which yields

$$
0=N[g]=\beta_{1} H_{1} e^{\omega_{1} P}+\beta_{2} H_{2} e^{\omega_{2} P}+\beta_{3} H_{3} e^{\omega_{3} P}, \quad \beta_{j}, \omega_{j} \in \mathbf{C}^{*},
$$

in which the $\omega_{j}$ are pairwise distinct, while $P^{\prime}$ and the $H_{j}$ are rational at infinity and $H_{j} e^{\omega_{j} P}=N\left[e^{\omega_{j} P} / P^{\prime}\right]$. This forces each $H_{j}$ to vanish identically, so that the equation $N[y]=0$ has three pairwise distinct exponential parts for its solutions, which is a contradiction.

\section{Two lemmas concerning trivial exponential parts}

If at least one of the three exponential parts arising from the equation $N[y]=0$ is trivial (that is, the zero polynomial), then it is not necessarily the case that $N[y]=0$ will have two linearly independent solutions decaying in the same sector, so that a second order equation (3.4) may not be available. The approach to this case will combine Lemma 3.1 with some ideas from [2].

Lemma 16.1. Assume that two exponential parts $\kappa_{1}, \kappa_{2}$ arising from the equation $N[y]=0$ are such that $\kappa_{2}$ is the zero polynomial, while

$$
\kappa_{1} \prec 0 \quad \text { or } \quad 0 \prec \kappa_{1}
$$

on a ray $\arg z=\theta$. Let the operators $L, M$ have canonical formal solutions with exponential parts as in (14.1). Then the exponential parts for $M$ are as in (14.2), while

$$
s_{j}=q_{j} \text { for each } j
$$

and the following additional conclusions hold. If $\kappa_{1} \prec 0$ in (16.1) then $\lambda=1$ and (16.3) $q_{1}=s_{1}=q_{2}+\kappa_{1}, \quad \ldots, \quad q_{k-1}=s_{k-1}=q_{k}+\kappa_{1}, \quad q_{k}=s_{k}=q_{1}-(k-1) \kappa_{1}$. If $0 \prec \kappa_{1}$ in (16.1) then $\lambda=k$ and

$$
q_{1}=s_{1}=q_{k}-(k-1) \kappa_{1}, \quad q_{2}=s_{2}=q_{1}+\kappa_{1}, \ldots, \quad q_{k}=s_{k}=q_{k-1}+\kappa_{1} .
$$

Proof. First observe that $N[y]=0$ has two canonical formal solutions which are free of logarithms and have exponential parts 0 and $\kappa_{1}$ respectively. Thus (14.2) and (16.2) hold by Lemma 7.1. Assume that $\kappa_{1} \prec 0$ in (16.1). If $\lambda \neq 1$ then an exponential part $q_{1}+\kappa_{1}$ occurs in the list (14.2), but this term, in view of (16.1), cannot be realised as $q_{j}$ for any $j$, contradicting (16.2). Now suppose that $s_{k} \neq q_{1}-(k-1) \kappa_{1}$; then $s_{k}=q_{k}+\kappa_{1}$, again contradicting (16.2).

Now assume that $0 \prec \kappa_{1}$ in (16.1). Then $\lambda$ must be $k$, since otherwise an exponential part $q_{k}+\kappa_{1}$ occurs in (14.2), contradicting (16.2). Moreover, $q_{1}=s_{1}=$ $q_{k}-(k-1) \kappa_{1}$, because the contrary case forces $s_{1}=q_{1}+\kappa_{1}$, which again contradicts (16.2).

Lemma 16.2. If there exists a ray $\arg z=\theta$ on which the three exponential parts arising from the equation $N[y]=0$ satisfy (11.2), then $\kappa_{3}=-\kappa_{1}$. 
Proof. Assuming the existence of such a ray, let the operators $L, M$ have exponential parts as in (14.1). Now (16.3) and (16.4) yield

$$
q_{1}=s_{1}=q_{k}-(k-1) \kappa_{3}, \quad q_{k}=s_{k}=q_{1}-(k-1) \kappa_{1}, \quad \kappa_{3}=-\kappa_{1} .
$$

\section{The case where (11.2) holds}

This section will deal with the case where there exists a ray for which conclusion (11.2) arises in Lemma 11.2. In this situation Lemma 16.2 makes it possible to assume that the exponential parts for $N[y]=0$ are $P, 0$ and $-P$, where $P$ is a polynomial in $z$ of positive degree $\rho$. Hence $N[y]=0$ has canonical formal solutions which are free of logarithms and satisfy

$$
u_{1}(z)=z^{\eta_{1}} e^{P(z)}(1+\ldots), \quad u_{2}(z)=z^{\eta_{2}}(1+\ldots), \quad u_{3}(z)=z^{\eta_{3}} e^{-P(z)}(1+\ldots) .
$$

Since $N[g]=0$, the order of growth of $g^{k}=f / F$ is $\rho\left(g^{k}\right)=\rho$. Choose a ray $\arg z=\theta_{0}$ on which $\operatorname{Re} P(z)=O\left(|z|^{\rho-1}\right)$ as $|z| \rightarrow \infty$, such that $f$ has a sequence of poles (and so $g$ has a sequence of simple zeros) tending to $\infty$ in the sector $\left|\arg z-\theta_{0}\right| \leq \pi / 2 \rho$. Take a sector $\Sigma$ given by $\left|\arg z-\theta_{0}\right| \leq \pi / \rho-\delta_{1}$, where $\delta_{1}$ is small and positive, and write

$$
g=U_{1}+U_{2}+U_{3}, \quad U_{j}=b_{j} \phi_{j}, \quad b_{j} \in \mathbf{C}, \quad \phi_{j} \sim u_{j},
$$

in which the $\phi_{j}$ are analytic solutions on $\Sigma$, and the last relation holds in the sense of asymptotic series, as in Section 4 . Here the fact that the asymptotics for $N[y]=0$ may be extended to hold in $\Sigma$ follows from the work of Jurkat [16]: in the present case, where the exponential parts are $P, 0$ and $-P$, it is relatively simple to establish, using the Phragmén-Lindelöf principle. Since $g$ has infinitely many zeros in $\Sigma$, at least two of the $b_{j}$, and so at least one of $b_{1}$ and $b_{3}$, must be non-zero. By replacing $P$ by $-P$, it may be assumed that $b_{1} \neq 0$.

Now take a ray $\arg z=\theta$ lying in $\Sigma$, on which $P \prec 0 \prec-P$, and apply Lemma 16.1 with $\kappa_{1}=P$. It follows from (16.2) and (16.3) that

$q_{2}=q_{1}-P, \quad q_{3}=q_{2}-P=q_{1}-2 P, \quad \ldots, \quad q_{k}=q_{k-1}-P=q_{1}-(k-1) P, \quad s_{j}=q_{j}$, and so, by (7.2),

$$
0=q_{1}+\ldots+q_{k}=k q_{1}-\left(\frac{k(k-1)}{2}\right) P, \quad q_{1}=\left(\frac{k-1}{2}\right) P, \quad q_{k}=-\left(\frac{k-1}{2}\right) P .
$$

Hence the equations $L[y]=0, M[y]=0$ have canonical formal solutions

$$
f_{j}(z)=z^{\lambda_{j}} e^{q_{j}(z)}(1+\ldots), \quad w_{j}(z)=z^{\mu_{j}} e^{q_{j}(z)}(1+\ldots),
$$

respectively, in which the $q_{j}$ are pairwise distinct. Since $a_{k-1}=A_{k-1}$, Lemma 4.2 implies that

$$
\lambda_{1}+\ldots+\lambda_{k}=\mu_{1}+\ldots+\mu_{k} .
$$

Write $v_{j}=V\left[u_{j}\right]$. By Lemmas 7.1 and 16.1, $u_{1}, u_{3}$ annihilate $f_{1}, f_{k}$ respectively, and (17.4) gives

$$
\frac{v_{1}(z)}{u_{1}(z)}=-\frac{f_{1}^{\prime}(z)}{f_{1}(z)}=\widehat{c}_{1} z^{\rho-1}+\ldots, \quad \frac{v_{3}(z)}{u_{3}(z)}=-\frac{f_{k}^{\prime}(z)}{f_{k}(z)}=\widehat{c}_{3} z^{\rho-1}+\ldots,
$$


where $\widehat{c}_{1}, \widehat{c}_{3}$ are non-zero constants. It follows from (5.16) and (17.1) that $v_{2} / u_{2}$ is given by a (possibly vanishing) formal series in descending integer powers of $z$ of the form

$$
\frac{v_{2}(z)}{u_{2}(z)}=\alpha(z) \frac{u_{2}^{\prime \prime}(z)}{u_{2}(z)}+\beta(z) \frac{u_{2}^{\prime}(z)}{u_{2}(z)}+\gamma(z)=c_{N} z^{N}+\ldots
$$

Lemma 17.1. The integer $k$ is at least 4.

Proof. Suppose that $k=3$; then (17.3) and (17.4) lead to

$$
q_{1}=P, \quad q_{2}=0, \quad q_{3}=-P .
$$

Now write, using (17.1), (17.5) and (17.7),

$$
\begin{aligned}
f_{3}^{\prime}(z) u_{1}(z)+f_{3}(z) v_{1}(z) & =f_{3}(z) u_{1}(z)\left(\frac{f_{3}^{\prime}(z)}{f_{3}(z)}-\frac{f_{1}^{\prime}(z)}{f_{1}(z)}\right) \\
& =z^{\lambda_{3}+\eta_{1}}(1+\ldots)\left(\frac{f_{3}^{\prime}(z)}{f_{3}(z)}-\frac{f_{1}^{\prime}(z)}{f_{1}(z)}\right)
\end{aligned}
$$

and

$$
\begin{aligned}
f_{1}^{\prime}(z) u_{3}(z)+f_{1}(z) v_{3}(z) & =f_{1}(z) u_{3}(z)\left(\frac{f_{1}^{\prime}(z)}{f_{1}(z)}-\frac{f_{3}^{\prime}(z)}{f_{3}(z)}\right) \\
& =z^{\lambda_{1}+\eta_{3}}(1+\ldots)\left(\frac{f_{1}^{\prime}(z)}{f_{1}(z)}-\frac{f_{3}^{\prime}(z)}{f_{3}(z)}\right) .
\end{aligned}
$$

Each of these is a formal solution of $M[y]=0$, with zero exponential part, and so a constant multiple of $w_{2}$. But this implies that $\lambda_{1}+\eta_{3}=\lambda_{3}+\eta_{1}$ and $f_{3}^{\prime} u_{1}+f_{3} v_{1}=$ $-\left(f_{1}^{\prime} u_{3}+f_{1} v_{3}\right)$, so that $f_{1} u_{3}=f_{3} u_{1}$, which leads in turn to

$$
\frac{V\left[u_{3}\right]}{u_{3}}-\frac{V\left[u_{1}\right]}{u_{1}}=\frac{f_{1}^{\prime}}{f_{1}}-\frac{f_{3}^{\prime}}{f_{3}}=\frac{u_{1}^{\prime}}{u_{1}}-\frac{u_{3}^{\prime}}{u_{3}}, \quad u_{1} V\left[u_{3}\right]-u_{3} V\left[u_{1}\right]=u_{3} u_{1}^{\prime}-u_{1} u_{3}^{\prime} .
$$

Hence (12.1) holds, with $g_{1}=u_{1}, g_{2}=u_{3}$ and $d=1$. Thus the hypotheses of Lemma 12.2 are satisfied, so that $d \neq(k-1) / 2=1$, a contradiction. 0 :

Lemma 17.2. One of the following two conclusions holds, in which $\rho=\operatorname{deg} P>$

(A) $\eta_{2}=-N \leq-\rho$ and $\eta_{1}+\eta_{3}=-2(\rho-1)$;

(B) $\eta_{1}+\eta_{3}-2 \eta_{2}=0$ and $f$ has order of growth $\rho$.

Proof. (17.3) and (17.7) show that, for $j=2, \ldots, k$, the term

$$
f_{j}^{\prime} u_{1}+f_{j} v_{1}=f_{j} u_{1}\left(\frac{f_{j}^{\prime}}{f_{j}}-\frac{f_{1}^{\prime}}{f_{1}}\right)
$$

is a canonical formal solution of $M[y]=0$ with exponential part $q_{j}+P=q_{j-1}$, and so is a constant multiple of $w_{j-1}$. This delivers, using (17.3), (17.5) and (17.6),

$$
\begin{aligned}
& \mu_{1}=\lambda_{2}+\eta_{1}+\rho-1, \quad \ldots, \quad \mu_{k-1}=\lambda_{k}+\eta_{1}+\rho-1, \\
& \mu_{k}=\lambda_{1}-(k-1)\left(\eta_{1}+\rho-1\right) .
\end{aligned}
$$

In the same way, for $j=1, \ldots, k-1$, the term $f_{j}^{\prime} u_{3}+f_{j} v_{3}$ has exponential part $q_{j}-P=q_{j+1}$, and so is a constant multiple of $w_{j+1}$, which yields

$$
\begin{aligned}
& \mu_{2}=\lambda_{1}+\eta_{3}+\rho-1, \quad \ldots, \quad \mu_{k}=\lambda_{k-1}+\eta_{3}+\rho-1, \\
& \mu_{1}=\lambda_{k}-(k-1)\left(\eta_{3}+\rho-1\right) .
\end{aligned}
$$


Suppose first that $N \geq \rho$ and $c_{N} \neq 0$ in (17.8). In this case (17.3) and (17.5) show that $u_{2}$ cannot annihilate any of the $f_{j}$, and that each $f_{j}^{\prime} u_{2}+f_{j} v_{2}$ is a canonical formal solution of $M[y]=0$ with exponential part $q_{j}$, and so a constant multiple of $w_{j}$. This implies in view of (17.6) that

$$
\mu_{j}=\lambda_{j}+\eta_{2}+N \quad(j=1, \ldots, k), \quad \eta_{2}=-N .
$$

Moreover, (17.9) and (17.10) now lead to

$$
\begin{aligned}
& \lambda_{k}=\mu_{k}=\lambda_{1}-(k-1)\left(\eta_{1}+\rho-1\right), \quad \lambda_{1}=\mu_{1}=\lambda_{k}-(k-1)\left(\eta_{3}+\rho-1\right), \\
& \eta_{1}+\rho-1=-\left(\eta_{3}+\rho-1\right),
\end{aligned}
$$

so that $\eta_{1}+\eta_{3}=-2(\rho-1)$ and conclusion (A) holds.

Now suppose that $N \leq \rho-1$ in (17.8): this case will lead to conclusion (B), and encompasses the possibility that $v_{2} / u_{2}$ vanishes identically. The first step is to show that the order of growth of $f$ is $\rho$. Since $g^{k}$ has order $\rho$ it follows from (5.1) that the order of $f$ is at least $\rho$. It suffices to show that in (5.16) the coefficients (which are rational at infinity) satisfy

$$
\alpha(z)=O\left(|z|^{1-\rho}\right), \quad \beta(z)=O(1), \quad \gamma(z)=O\left(|z|^{\rho-1}\right) \quad \text { as } z \rightarrow \infty,
$$

because if this can be established then $\rho(f) \leq \rho$ follows from (5.16), the WimanValiron theory [12] applied to $1 / f$, and standard estimates [8] for logarithmic derivatives of $g^{k}$ and $g$.

To prove (17.11) use (17.1), (17.7) and (17.8) to write

$$
\begin{aligned}
& \alpha(z) P^{\prime}(z)^{2}\left(1+O\left(z^{-1}\right)\right)+\beta(z) P^{\prime}(z)\left(1+O\left(z^{-1}\right)\right)+\gamma(z)=\frac{v_{1}(z)}{u_{1}(z)}=O\left(z^{\rho-1}\right), \\
& \alpha(z) P^{\prime}(z)^{2}\left(1+O\left(z^{-1}\right)\right)-\beta(z) P^{\prime}(z)\left(1+O\left(z^{-1}\right)\right)+\gamma(z)=\frac{v_{3}(z)}{u_{3}(z)}=O\left(z^{\rho-1}\right), \\
& \alpha(z) O\left(z^{-2}\right)+\beta(z) O\left(z^{-1}\right)+\gamma(z)=\frac{v_{2}(z)}{u_{2}(z)}=O\left(z^{N}\right)=O\left(z^{\rho-1}\right) .
\end{aligned}
$$

Here $O\left(z^{\omega}\right)$ denotes any formal series in descending integer powers of $z$ with leading power at most $\omega \in \mathbf{Z}$. Eliminating $\gamma$ via the last equation yields

$$
\begin{aligned}
& \alpha(z) P^{\prime}(z)^{2}\left(1+O\left(z^{-1}\right)\right)+\beta(z) P^{\prime}(z)\left(1+O\left(z^{-1}\right)\right)=O\left(z^{\rho-1}\right), \\
& \alpha(z) P^{\prime}(z)^{2}\left(1+O\left(z^{-1}\right)\right)-\beta(z) P^{\prime}(z)\left(1+O\left(z^{-1}\right)\right)=O\left(z^{\rho-1}\right),
\end{aligned}
$$

and now (17.11) follows from Cramer's rule.

Next, since $N \leq \rho-1,(17.3)$ and (17.8) give pairwise distinct $\widehat{d}_{j} \in \mathbf{C}$ with

$$
\frac{f_{j}^{\prime}(z)}{f_{j}(z)}+\frac{v_{2}(z)}{u_{2}(z)}=\widehat{d}_{j} z^{\rho-1}+\ldots
$$

If $\widehat{d}_{j} \neq 0$ then $f_{j}^{\prime} u_{2}+f_{j} v_{2}$ is again a canonical formal solution of $M[y]=0$ with exponential part $q_{j}$, and so a constant multiple of $w_{j}$. Since $k \geq 4$, this implies in view of (17.5) that

$$
\mu_{j}=\lambda_{j}+\eta_{2}+\rho-1
$$

for $j=1$ and $j=2$, or for $j=k-1$ and $j=k$. If (17.12) holds for $j=1$ and $j=2$ then (17.9), (17.10) and (17.12) give

$$
\mu_{1}=\lambda_{1}+\eta_{2}+\rho-1=\lambda_{2}+\eta_{1}+\rho-1, \quad \mu_{2}=\lambda_{2}+\eta_{2}+\rho-1=\lambda_{1}+\eta_{3}+\rho-1,
$$


from which it follows that

$$
\eta_{1}-\eta_{2}=\lambda_{1}-\lambda_{2}=\eta_{2}-\eta_{3}, \quad \eta_{1}+\eta_{3}-2 \eta_{2}=0 .
$$

Similarly, if (17.12) holds for $j=k-1$ and $j=k$, then (17.9), (17.10) and (17.12) give

$\mu_{k}=\lambda_{k}+\eta_{2}+\rho-1=\lambda_{k-1}+\eta_{3}+\rho-1, \quad \mu_{k-1}=\lambda_{k-1}+\eta_{2}+\rho-1=\lambda_{k}+\eta_{1}+\rho-1$,

which delivers

$$
\eta_{1}-\eta_{2}=\lambda_{k-1}-\lambda_{k}=\eta_{2}-\eta_{3}, \quad \eta_{1}+\eta_{3}-2 \eta_{2}=0 .
$$

Lemma 17.3. If $b_{3}=0$ in (17.2) then $f$ satisfies the conclusion of Proposition 10.1 .

Proof. Using (5.16) write, on $\Sigma$,

$$
\begin{aligned}
g & =U_{1}+U_{2}, \quad-\frac{f^{\prime}}{f}=\frac{V[g]}{g}=\frac{V\left[U_{1}\right]+V\left[U_{2}\right]}{U_{1}+U_{2}}=\frac{V\left[U_{1}\right] / U_{2}+V\left[U_{2}\right] / U_{2}}{e^{\Phi}+1}, \\
e^{\Phi} & =\frac{U_{1}}{U_{2}} .
\end{aligned}
$$

A zero of $g$ arises wherever $U_{1} / U_{2}=e^{\Phi}=-1$, and the multiplicity of the pole of $f$ at such a point is

$$
m_{0}=\frac{V\left[U_{1}\right] / U_{1}-V\left[U_{2}\right] / U_{2}}{\Phi^{\prime}} .
$$

By (17.1) and (17.2), the function $\zeta=(1 / \pi i) \Phi=(1 / \pi i) \log U_{1} / U_{2}$ maps the sector $\Sigma$ univalently onto a region containing a half-plane $\pm \operatorname{Re} \zeta>M_{1} \in \mathbf{R}$, and (17.14) holds wherever $\zeta$ is an odd integer. Thus (17.1), (17.2), (17.7) and Lemma 3.1 give a polynomial $Q^{*}$ such that

$$
\frac{V\left[U_{1}\right]}{U_{1}}-\frac{V\left[U_{2}\right]}{U_{2}}=Q^{*}(\Phi) \Phi^{\prime}, \quad U_{2} V\left[U_{1}\right]-U_{1} V\left[U_{2}\right]=Q^{*}(\Phi)\left(U_{2} U_{1}^{\prime}-U_{1} U_{2}^{\prime}\right)
$$

Suppose first that $Q^{*}(\Phi)$ is rational at infinity in (17.15). Then it follows from Lemma 12.1 that $U_{1}$ and $U_{2}$ solve a second order equation (3.4) with $E_{1}$ and $E_{0}$ rational at infinity, and so does $g$, by (17.13), contradicting Lemma 10.1(C).

It may therefore be assumed henceforth that $Q^{*}$ is non-constant. Then (17.14) and (17.15) show that the multiplicity $m_{0}(z)$ of a pole $z \in \Sigma$ of $f$ tends to $\infty$ as $z \rightarrow \infty$, faster than $|z|^{\rho_{1}}$ for some $\rho_{1}>0$. Since the zeros of $g=U_{1}+U_{2}$ in $\Sigma$ have exponent of convergence $\rho$, this is incompatible with Case B of Lemma 17.2. Hence Case A of Lemma 17.2 must hold, and so $\left(\eta_{1}-\eta_{2}\right)-\left(\eta_{2}-\eta_{3}\right)=\eta_{1}+\eta_{3}-2 \eta_{2}$ is a positive integer.

Furthermore, the left-hand side of (17.15) has a meromorphic continuation along any path in $\Omega\left(r_{1}\right)$, as has $\Phi^{\prime}$, but if a continuation of $U_{1} / U_{2}$ has a zero or pole at some $z_{0}$ then $\Phi(z)=\log U_{1}(z) / U_{2}(z)$ behaves like $m_{1} \log \left(z-z_{0}\right)$ as $z \rightarrow z_{0}$, for some $m_{1} \in \mathbf{Z} \backslash\{0\}$. Therefore (17.15) implies that $e^{\Phi}=U_{1} / U_{2}$ continues without poles or zeros in $\Omega\left(r_{1}\right)$, and so any zeros of continuations of $U_{1}$ and $U_{2}$ are shared.

Take any sector $\Sigma^{*}$ given by $\left|\arg z-\theta^{*}\right| \leq \pi / \rho-\delta_{1}$, where $\operatorname{Re} P\left(r e^{i \theta^{*}}\right)=O\left(r^{\rho-1}\right)$ as $r \rightarrow \infty$, let $\widetilde{U}_{1}, \widetilde{U}_{2}$ be continuations of $U_{1}, U_{2}$ to $\Sigma^{*}$, and write

$$
\widetilde{U}_{1}=d_{1} \psi_{1}+d_{2} \psi_{2}+d_{3} \psi_{3}, \quad \widetilde{U}_{2}=e_{1} \psi_{1}+e_{2} \psi_{2}+e_{3} \psi_{3}, \quad d_{j}, e_{j} \in \mathbf{C},
$$


on $\Sigma^{*}$, in which the $\psi_{j}$ are analytic solutions of $N[y]=0$ which satisfy, as $z \rightarrow \infty$ on $\Sigma^{*}$

$$
\begin{aligned}
& \psi_{1}(z)=z^{\eta_{1}} e^{P(z)}(1+o(1)), \quad \psi_{2}(z)=z^{\eta_{2}}(1+o(1)), \\
& \psi_{3}(z)=z^{\eta_{3}} e^{-P(z)}(1+o(1)) .
\end{aligned}
$$

Suppose that $\widetilde{U}_{1}$ and $\widetilde{U}_{2}$ have a sequence $\zeta_{\mu} \rightarrow \infty$ of common zeros in $\Sigma^{*}$. The matrix with rows $\left(d_{1}, d_{2}, d_{3}\right)$ and $\left(e_{1}, e_{2}, e_{3}\right)$ has rank 2 , since $U_{1}$ and $U_{2}$ are linearly independent, and so Cramer's rule gives $e_{4}, e_{5} \in \mathbf{C}$ and a permutation $\left(j, j^{\prime}, j^{\prime \prime}\right)$ of $(1,2,3)$ such that

$$
\psi_{j^{\prime}}\left(\zeta_{\mu}\right)=e_{4} \psi_{j}\left(\zeta_{\mu}\right), \quad \psi_{j^{\prime \prime}}\left(\zeta_{\mu}\right)=e_{5} \psi_{j}\left(\zeta_{\mu}\right) \quad \text { as } \mu \rightarrow \infty .
$$

Here $e_{4} e_{5} \neq 0$, as $\psi_{j}\left(\zeta_{\mu}\right) \neq 0$ for large $\mu$. But this gives a contradiction, since the fact that $\left(\eta_{1}-\eta_{2}\right)-\left(\eta_{2}-\eta_{3}\right)$ is positive implies that $\psi_{2}\left(\zeta_{\mu}\right) / \psi_{3}\left(\zeta_{\mu}\right)=o\left(\left|\psi_{1}\left(\zeta_{\mu}\right) / \psi_{2}\left(\zeta_{\mu}\right)\right|\right)$ as $\mu \rightarrow \infty$.

It follows that $U_{1}$ and $U_{2}$ continue without zeros in some annulus $\Omega\left(r^{*}\right)$. Lemma 3.2 shows that there exists $\rho_{2}>0$ such that any continuation of $U_{2}$ to any sector in $\Omega\left(r^{*}\right)$ satisfies $\log \left|U_{2}(z)\right|=O\left(|z|^{\rho_{2}}\right)$ as $z \rightarrow \infty$ there. Take a sector $\Sigma^{* *}$ given by $\theta_{1}<\arg z<\theta_{2}$, where these $\theta_{j}$ are such that no $\theta \in\left[\theta_{1}, \theta_{2}\right]$ has $\operatorname{Re} P\left(r e^{i \theta}\right)=O\left(r^{\rho-1}\right)$ as $r \rightarrow \infty$. For any continuation of $U_{2}$ to $\Sigma^{* *}$ there exist $P^{*} \in\{-P, 0, P\}$ and a matching $\eta^{*} \in\left\{\eta_{1}, \eta_{2}, \eta_{3}\right\}$ such that $U_{2}(z) \sim c z^{\eta^{*}} \exp \left(P^{*}(z)\right)$ as $z \rightarrow \infty$ in $\Sigma^{* *}$. Since $U_{2}(z) \sim c z^{\eta_{2}}$ as $z \rightarrow \infty$ in $\Sigma$, repeated application of the Phragmén-Lindelöf principle to the continuations of $U_{2}(z) z^{-\eta_{2}}$ or its reciprocal shows that $P^{*}=0$, and so $\eta^{*}=\eta_{2}$. Examining (17.16) in the light of (17.17), first on a subsector of $\Sigma^{*}$ on which $e^{P}$ is large and subsequently on a subsector where $e^{-P}$ is large, forces $0=e_{1}=e_{3}$. Choosing $\Sigma^{*}=\Sigma$ gives $e_{0} \in \mathbf{C}$ such that $z^{e_{0}} U_{2}(z)$ is analytic and zero-free of finite order of growth in some annulus $\Omega\left(r^{* *}\right)$. This, coupled with almost identical reasoning applied to $U_{1}$, shows that $U_{1}^{\prime} / U_{1}, U_{2}^{\prime} / U_{2}$ and $\Phi^{\prime}$ are rational at infinity, as is $Q^{*}(\Phi)$ by (17.15), and this case has already been dealt with.

Assume henceforth that $b_{1} b_{3} \neq 0$ in (17.2), and write this formula for $g$ as

$$
\begin{aligned}
g & =A e^{-P}\left(\left(e^{P}-B\right)^{2}-C^{2}\right), \quad U_{1}=A e^{P}, \\
U_{2} & =-2 A B, \quad U_{3}=A\left(B^{2}-C^{2}\right) e^{-P} .
\end{aligned}
$$

By (17.1), this initially formal expression for $g$ results in, as $z \rightarrow \infty$ in $\Sigma$,

$$
\begin{aligned}
A(z) & =b_{1} z^{\eta_{1}} \chi_{1}(z), \quad B(z)=-\frac{b_{2}}{2 b_{1}} z^{\eta_{2}-\eta_{1}} \chi_{2}(z), \\
B(z)^{2}-C(z)^{2} & =\frac{b_{3}}{b_{1}} z^{\eta_{3}-\eta_{1}} \chi_{3}(z), \quad \chi_{j}(z)=1+o(1) .
\end{aligned}
$$

Here the $\chi_{j}$ have asymptotic series on $\Sigma$ in descending integer powers of $z$ and, by Lemma 17.2, $\eta_{3}-\eta_{1}-2\left(\eta_{2}-\eta_{1}\right)=\eta_{1}+\eta_{3}-2 \eta_{2}$ is a non-negative even integer. Evidently $A, B$ and $E=C^{2}$ are analytic on $\Sigma$, and $E$ does not vanish identically, since zeros of $g$ are simple. Furthermore, it is clear from (17.18) that, at a zero of $g$ in $\Sigma$,

$$
\left(e^{P}-B\right)^{2}=E=C^{2}, \quad g^{\prime}=A e^{-P}\left(2\left(e^{P}-B\right)\left(P^{\prime} e^{P}-B^{\prime}\right)-E^{\prime}\right) .
$$

Lemma 17.4. Let $d= \pm 1$. Then there exist $r_{2}>0$ and $\sigma_{d}, \tau_{d} \in \mathbf{C}^{*}$, as well as $\gamma_{d}, \zeta_{d} \in \mathbf{C}$, such that $B+d C$ is analytic on $\Sigma \cap \Omega\left(r_{2}\right)$ and

$$
C(z)^{2}=\sigma_{d} z^{\gamma_{d}} \psi_{1}(z), \quad \psi_{1}(z)=1+o(1),
$$


and

$$
B(z)+d C(z)=\tau_{d} z^{\zeta_{d}} \psi_{2}(z), \quad \psi_{2}(z)=1+o(1)
$$

as $z \rightarrow \infty$ in $\Sigma$, in which the $\psi_{j}(z)$ have asymptotic series on $\Sigma$ in descending integer powers of $z^{1 / 2}$. Furthermore, if conclusion (A) of Lemma 17.2 holds, then $\gamma_{d}=\eta_{3}-\eta_{1}$.

Proof. Note first that $B+d C$ does not vanish identically, since $B^{2}-C^{2}$ does not. All conclusions of the lemma clearly follow from (17.19) if $b_{2}=0$ or $\eta_{3}-\eta_{1}-$ $2\left(\eta_{2}-\eta_{1}\right)>0$, and in particular if conclusion (A) of Lemma 17.2 holds.

Assume therefore that $b_{2} \neq 0$ and $\eta_{3}-\eta_{1}=2\left(\eta_{2}-\eta_{1}\right)$. Then (17.19) implies that $\widetilde{C}(z)=C(z)^{2} z^{2\left(\eta_{1}-\eta_{2}\right)}$ has an asymptotic series on $\Sigma$ in descending non-positive integer powers of $z$. If this asymptotic series for $\widetilde{C}(z)$ vanishes identically then, by making $\Sigma$ slightly narrower if necessary, it may be assumed that $E(z)=C(z)^{2}$ and $E^{\prime}(z)$ both tend to zero in $\Sigma$ transcendentally fast, that is, faster than any negative power of $z$, but $f$ still has infinitely many poles there. This implies using (5.2) and (17.20) that if $M_{1}$ is a positive integer and $z$ is a pole of $f$ of multiplicity $m_{0}(z)$ in $\Sigma$, with $|z|$ large, then

$$
g(z)=0, \quad e^{P(z)}=B(z)+O\left(|z|^{-2 M_{1}}\right), \quad g^{\prime}(z)=O\left(|z|^{-M_{1}}\right), \quad|z|^{M_{1}}=O\left(m_{0}(z)\right),
$$

which is a contradiction since $f$ has finite order. Hence there must exist an integer $m_{1} \leq 0$ such that $(17.21)$ holds with $\gamma_{d}=2\left(\eta_{2}-\eta_{1}\right)+m_{1}$, in which $\psi_{1}(z)$ has an asymptotic series in descending integer powers of $z$. It is now clear from (17.19) and $(17.21)$ that $\widetilde{B}(z)=(B(z)+d C(z)) z^{\eta_{1}-\eta_{2}}$ has an asymptotic series on $\Sigma$ in descending integer powers of $z^{1 / 2}$; thus (17.22) holds unless this series for $\widetilde{B}(z)$ vanishes identically, in which case $B(z)+d C(z)$ tends to zero transcendentally fast on $\Sigma$, and so does $B(z)^{2}-C(z)^{2}$, by the second equation of (17.19), which forces $b_{3}=0$ in (17.19), contrary to assumption.

Lemma 17.5. For $d= \pm 1$ there exists a polynomial $Q_{d} \not \equiv 0$ such that

$$
\left[2 d C A\left(P^{\prime}-\frac{B^{\prime}+d C^{\prime}}{B+d C}\right)\right]^{-k}=Q_{d}(P-\log (B+d C)) \text {. }
$$

Proof. The function $g$ has a zero in $\Sigma$ wherever $e^{P}=B+d C$, and at such a zero (17.20) gives

$$
\begin{aligned}
g^{\prime} & =A e^{-P}\left(2 d C\left(P^{\prime} e^{P}-B^{\prime}\right)-2 C C^{\prime}\right)=2 d C A e^{-P}\left(P^{\prime} e^{P}-B^{\prime}-d C^{\prime}\right) \\
& =2 d C A\left(P^{\prime}-\frac{B^{\prime}+d C^{\prime}}{B+d C}\right) .
\end{aligned}
$$

Here (17.22) shows that $\zeta=(1 / 2 \pi i)(P(z)-\log (B(z)+d C(z)))$ maps a subdomain of $\Sigma$ univalently onto a half-plane $\pm \operatorname{Re} \zeta>M_{1} \in \mathbf{R}$. Because (5.2) implies that $\left(g^{\prime}\right)^{-k}$ is integer-valued at each zero of $g$, and so at points where $\zeta$ is integer-valued, it follows from (17.19), (17.21) and Lemma 3.1 that a polynomial $Q_{d}$ exists as asserted.

Lemma 17.6. For $d= \pm 1$ the polynomial $Q_{d}$ in (17.23) is constant.

Proof. Assume that $Q_{d}$ is non-constant. Then it follows from (5.2), (17.22), (17.23) and (17.24) that the multiplicity $m_{0}(z)$ of the pole of $f$ at $z \in \Sigma$ tends to $\infty$ faster than some positive power of $|z|$ and, since the exponent of convergence of the zeros of $e^{P}-(B+d C)$ in $\Sigma$ is $\rho$, this implies that $N(r, f)$ has order greater than $\rho$, which is incompatible with conclusion (B) of Lemma 17.2. 
Hence conclusion (A) of Lemma 17.2 must hold. In view of (17.19) and Lemma 17.4, it follows that $\eta_{1}+\eta_{3}=-2(\rho-1)$ and $\gamma_{d}=\eta_{3}-\eta_{1}$, and that

$$
C(z) A(z) \sim c z^{\gamma_{d} / 2+\eta_{1}}=c z^{\left(\eta_{1}+\eta_{3}\right) / 2}=c z^{1-\rho}
$$

as $z \rightarrow \infty$ in $\Sigma$. But then the left-hand side of (17.23) is bounded as $z \rightarrow \infty$ in $\Sigma$, which is a contradiction.

Lemma 17.7. There exist a large positive $r_{3}$ and an analytic function $K$ such that

$$
\begin{aligned}
& K^{\prime}=\frac{1}{U_{1}}, \quad U_{1}=A e^{P}, \quad U_{2}=-2 A B=e_{3} U_{1} K, \\
& U_{3}=A\left(B^{2}-C^{2}\right) e^{-P}=e_{4} U_{1} K^{2},
\end{aligned}
$$

on $\Sigma \cap \Omega\left(r_{3}\right)$, where $e_{3}, e_{4} \in \mathbf{C}$ and $e_{4} \neq 0$.

Proof. Suppose first that $B \not \equiv 0$. Then (17.23) holds for $d=1$ and $d=-1$, with $Q_{1}$ and $Q_{-1}$ both constant by Lemma 17.6. Hence, by (17.22) and (17.23),

$$
C A\left(P^{\prime}-\frac{B^{\prime}+C^{\prime}}{B+C}\right), \quad C A\left(P^{\prime}-\frac{B^{\prime}-C^{\prime}}{B-C}\right)
$$

are both constant, and so identically equal. Thus $(B+C) /(B-C)$ must be constant and so must $B / C$. Now (17.18), (17.21), (17.23) and Lemma 17.6 yield, with $c \in \mathbf{C}^{*}$ as before,

$$
C A\left(P^{\prime}-\frac{C^{\prime}}{C}\right)=c, \quad C^{\prime}-P^{\prime} C=\frac{c}{A}, \quad C e^{-P}=c \int \frac{1}{A e^{P}}=c \int \frac{1}{U_{1}}=c K,
$$

from which (17.25) follows, using (17.18) again. On the other hand, if $B \equiv 0$ then the first equation of (17.26) still holds, by Lemma 17.6, and the formula for $U_{2}$ in (17.25) is trivially satisfied with $e_{3}=0$.

Lemma 17.8. The function $K$ of Lemma 17.7 continues meromorphically along any path in the annulus $\Omega\left(r_{3}\right)$, its continuations locally univalent. Moreover, all zeros of any continuation of $U_{1}$ into $\Omega\left(r_{3}\right)$ are simple poles of $K$.

Proof. Since $e_{4} \neq 0$ in (17.25), writing

$$
\Phi=K^{2}=\frac{U_{3}}{e_{4} U_{1}}, \quad \frac{1}{U_{1}^{2}}=\left(K^{\prime}\right)^{2}=\frac{\left(\Phi^{\prime}\right)^{2}}{4 \Phi},
$$

shows that $\Phi$ continues meromorphically along any path in $\Omega\left(r_{3}\right)$. Any zero of any continuation of $U_{1}$ is either simple or double, since $U_{1}$ solves $N[y]=0$, and must be a pole of $\Phi$, by (17.27). Comparing multiplicities in (17.27) excludes simple zeros of $U_{1}$, and double zeros of $U_{1}$ have to be triple poles of $\Phi^{\prime}$ and so double poles of $\Phi$. Furthermore, any zeros of any continuation of $\Phi$ must be double, again by (17.27). Thus $K=\Phi^{1 / 2}$ continues meromorphically along paths in $\Omega\left(r_{3}\right)$, and is locally univalent since $K^{\prime}(z)=1 / U_{1}(z) \neq 0$.

Again because $e_{4} \neq 0$ in (17.25), there exists a polynomial $Q_{2}$ of degree 2 such that (17.2) and continuation of $g$ into $\Omega\left(r_{3}\right)$ give $g=Q_{2}(K) / K^{\prime}$, whether or not $U_{2} \equiv 0$, where $K$ is as in Lemma 17.8. Hence $g=0$ forces $K=a$, where $Q_{2}(a)=0$, and so $g^{\prime}=Q_{2}^{\prime}(a)= \pm b$ for some $b \in \mathbf{C}^{*}$, by elementary properties of quadratics. It now follows using (5.2) that all poles of $f$ in $\Omega\left(r_{3}\right)$ have the same multiplicity, and $f$ satisfies the conclusion of Proposition 10.1. 


\section{The case of a repeated trivial exponential part}

There remains only one case to deal with, in which the equation $N[y]=0$ has two linearly independent formal solutions $g_{1}, g_{2}$ with trivial exponential part. The third exponential part $\kappa$ must be non-zero, by Lemma 11.1. Take a ray $\arg z=\theta_{0}$ on which $\kappa \prec 0$, and label the exponential parts arising from $L$ and $M$ to be consistent with (14.1) on $\arg z=\theta_{0}$. It then follows from Lemma 16.1 that the exponential parts $q_{j}$ for the equation $L[y]=0$ are pairwise distinct, and the same is true for $M[y]=0$, and so the formal solutions of these equations are free of logarithms. This implies that any formal solution $G$ of $N[y]=0$ is also free of logarithms; to see this, take a fundamental set of canonical formal solutions $f_{j}$ of $L[y]=0$, write $f_{j}^{\prime} G+f_{j} V[G]=w_{j}$, where the $w_{j}$ are formal solutions of $M[y]=0$, and solve for $G$.

Therefore $N[y]=0$ has linearly independent canonical formal solutions $g_{1}, g_{2}$ each having the form $g_{j}(z)=z^{m_{j}}(1+\ldots)$, with $m_{j} \in \mathbf{C}$. There exists a third canonical formal solution $g_{3}$, which has exponential part $\kappa$ and, by Lemma 7.1, annihilates some canonical formal solution $h_{\mu}$ of $L[y]=0$, with exponential part $q_{\mu}$ say. Consider the terms $R_{j}=V\left[g_{j}\right] / g_{j}$, for $j=1,2$; these are formal series in descending integer powers of $z$. Hence

$$
S_{j}=h_{\mu}^{\prime} g_{j}+h_{\mu} V\left[g_{j}\right]=h_{\mu} g_{j}\left(h_{\mu}^{\prime} / h_{\mu}+R_{j}\right)
$$

is a formal solution of $M[y]=0$, for $j=1,2$, and either is identically zero or has exponential part $q_{\mu}$. Since the exponential parts for $M$ are all different, $S_{1}$ and $S_{2}$ must be linearly dependent, and some non-trivial linear combination $g_{4}$ of $g_{1}$ and $g_{2}$ must annihilate $h_{\mu}$, as does $g_{3}$. Therefore $g_{3} V\left[g_{4}\right]=g_{4} V\left[g_{3}\right]$ and Lemma 12.2, with $d=0$, gives an equation (3.4) solved by $g_{3}$ and $g_{4}$. Furthermore, $g_{4}$ must be a canonical formal solution of $N[y]=0$; this is obvious unless $g_{4}=d_{1} g_{1}-d_{2} g_{2}$ with $d_{1}, d_{2} \in \mathbf{C}^{*}$, in which case

$$
d_{1} S_{1}=d_{2} S_{2}, \quad d_{1} g_{1}\left(h_{\mu}^{\prime} / h_{\mu}+R_{1}\right)=d_{2} g_{2}\left(h_{\mu}^{\prime} / h_{\mu}+R_{2}\right) .
$$

Thus $W\left(g_{3}, g_{4}\right)$ has non-zero exponential part, so that $E_{1}(\infty) \neq 0$ in $(3.4)$, and the conclusion of Proposition 10.1 follows from Lemma 12.2.

\section{References}

[1] Brannan, D. A., and W. K. Hayman: Research problems in complex analysis. - Bull. London Math. Soc. 21, 1989, 1-35.

[2] Brüggemann, F.: Proof of a conjecture of Frank and Langley concerning zeros of meromorphic functions and linear differential polynomials, - Analysis 12:1/2, 1992, 5-30.

[3] Frank, G.: Eine Vermutung von Hayman über Nullstellen meromorpher Funktionen. - Math. Zeit. 149, 1976, 29-36.

[4] Frank, G., and S. Hellerstein: On the meromorphic solutions of nonhomogeneous linear differential equations with polynomial coefficients. - Proc. London Math. Soc. (3) 53, 1986 , 407-428.

[5] Frank, G., W. Hennekemper, and G. Polloczek: Über die Nullstellen meromorpher Funktionen and ihrer Ableitungen. - Math. Ann. 225, 1977, 145-154.

[6] Frank, G., and J. K. Langley: Pairs of linear differential polynomials. - Analysis 19, 1999, 173-194.

[7] Gao, S., and J. K. LAngley: On the zeros of certain linear differential polynomials. - J. Math. Anal. Appl. 153, 1990, 159-178.

[8] Gundersen, G.: Estimates for the logarithmic derivative of a meromorphic function, plus similar estimates. - J. London Math. Soc. 37, 1988, 88-104. 
[9] Hardy, G. H., and E. M. Wright: The theory of numbers. - Oxford Univ. Press, 1938.

[10] Hayman, W.K.: Picard values of meromorphic functions and their derivatives. - Ann. of Math. 70, 1959, 9-42.

[11] Hayman, W. K.: Meromorphic functions. - Oxford at the Clarendon Press, 1964.

[12] Hayman, W. K.: The local growth of power series: a survey of the Wiman-Valiron method. - Canad. Math. Bull. 17, 1974, 317-358.

[13] Heckner, M.: On meromorphic functions and linear differential polynomials which have few zeros. - Mitt. Math. Sem. Giessen 211, 1992, 1-14.

[14] Hille, E.: Ordinary differential equations in the complex domain. - Wiley, New York, 1976.

[15] InCE, E. L.: Ordinary differential equations. - Dover, New York, 1956.

[16] JuRkat, W.: Meromorphe Differentialgleichungen. - Lecture Notes in Math. 637, Springer, Berlin, 1978.

[17] Langley, J. K.: An application of the Tsuji characteristic. - J. Fac. Sci. Uni. Tokyo 38, 1991, 299-318.

[18] Langley, J. K.: Proof of a conjecture of Hayman concerning $f$ and $f^{\prime \prime}$. - J. London Math. Soc. (2) 48, 1993, 500-514.

[19] Langley, J. K.: On second order linear differential polynomials. - Resultate der Mathematik $26,1994,51-82$.

[20] Langley, J. K.: Integer-valued analytic functions in a half-plane. - Comput. Methods Funct. Theory 7, 2007, 433-442.

[21] Langley, J. K.: Second order linear differential polynomials and real meromorphic functions. - Resultate der Mathematik 63, 2013, 151-169.

[22] Lutz, D. A.: Asymptotic behavior of solutions of linear systems of ordinary differential equations near an irregular singular point. - Amer. J. Math. 91, 1969, 95-105.

[23] Steinmetz, N.: On the zeros of $\left(f^{(p)}+a_{p-1} f^{(p-1)}+\ldots+a_{0} f\right) f$. - Analysis 7, 1987, 375-389.

[24] WAsow, W.: Asymptotic expansions for ordinary differential equations. - Dover, New York, 1987.

Received 14 March 2017 • Accepted 1 February 2018 